\title{
Dietary Supplement Laboratory Quality Assurance Program: Exercise I Final Report
}

\author{
Melissa M. Phillips \\ Catherine A. Rimmer \\ Laura J. Wood \\ Karen E. Murphy \\ Thomas W. Vetter
}


NISTIR 7955

\section{Dietary Supplement Laboratory Quality Assurance Program: Exercise I Final Report}

Melissa M. Phillips Catherine A. Rimmer

Laura J. Wood

Karen E. Murphy

Thomas W. Vetter

Chemical Sciences Division Material Measurement Laboratory

http://dx.doi.org/10.6028/NIST.IR.7955

August 2013

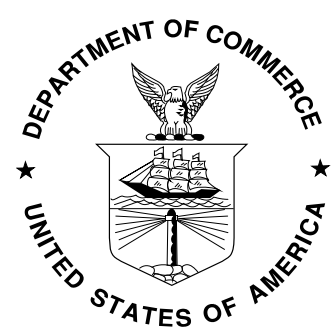

U.S. Department of Commerce

Penny Pritzker, Secretary

National Institute of Standards and Technology

Patrick D. Gallagher, Under Secretary of Commerce for Standards and Technology and Director 


\section{TABLE OF CONTENTS}

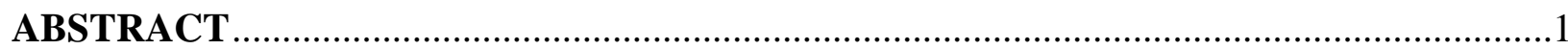

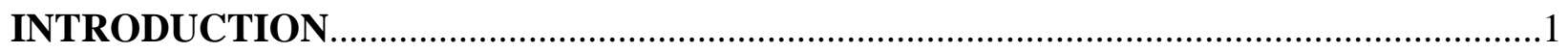

OVERVIEW OF DATA TREATMENT AND REPRESENTATION

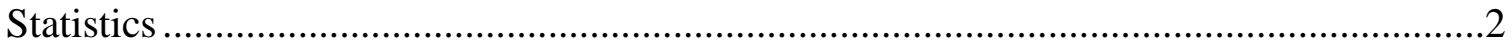

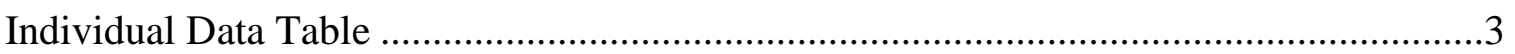

Summary Data Table ........................................................................................

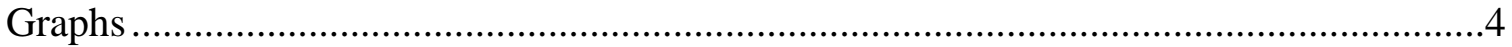

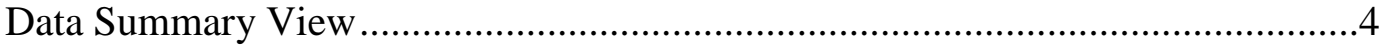

Sample/Control Comparison View .................................................................

TRACE NUTRITIONAL ELEMENTS IN FOODS AND SUPPLEMENTS

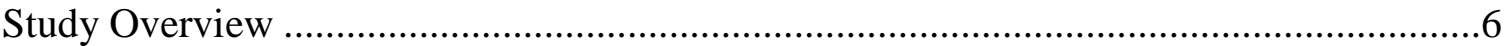

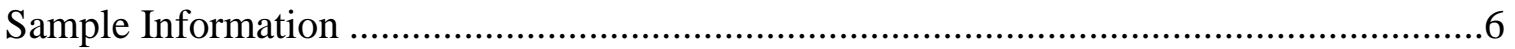

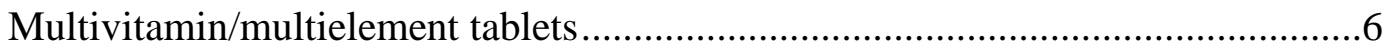

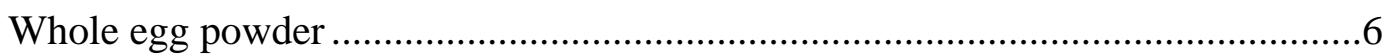

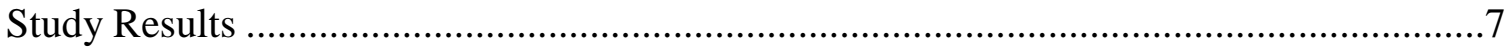

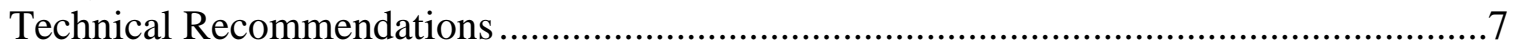

Table 1. Individual data table (NIST) for trace nutritional elements in foods and dietary supplements.

Table 2. Data summary table for chromium in foods and dietary supplements...............10

Table 3. Data summary table for molybdenum in foods and dietary supplements..........11

Table 4. Data summary table for selenium in foods and dietary supplements................12

Figure 1. Chromium in SRM 3280 Multivitamin/Multielement Tablets (method comparison data summary view - digestion method)........................................13

Figure 2. Chromium in whole egg powder (data summary view). ...............................14

Figure 3. Molybdenum in SRM 3280 Multivitamin/Multielement Tablets (data summary

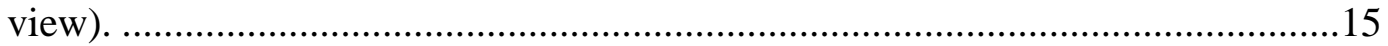

Figure 4. Molybdenum in whole egg powder (data summary view) ............................16

Figure 5. Selenium in SRM 3280 Multivitamin/Multielement Tablets (data summary

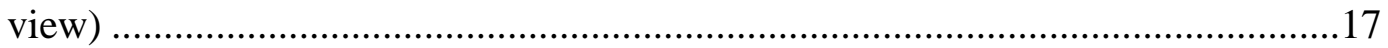

Figure 6. Selenium in whole egg powder (data summary view) .................................18

Figure 7. Chromium in whole egg powder and SRM 3280 Multivitamin/Multielement Tablets (sample/control comparison view) .....................................................19

Figure 8. Molybdenum in whole egg powder and SRM 3280 Multivitamin/Multielement Tablets (sample/control comparison view) ...................................................20

Figure 9. Selenium in whole egg powder and SRM 3280 Multivitamin/Multielement Tablets (sample/control comparison view) ....................................................21

TOXIC ELEMENTS (CD) IN FOODS AND SUPPLEMENTS

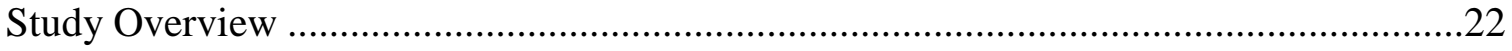

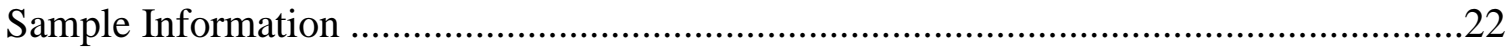

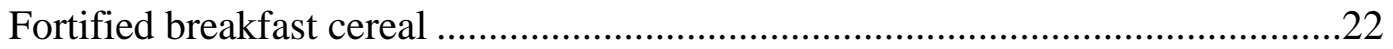




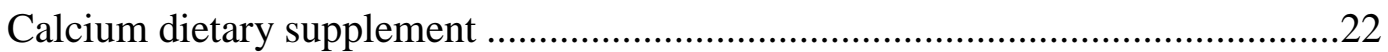

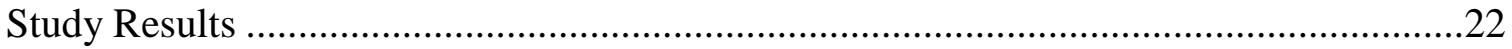

Technical Recommendations ...............................................................................23

Table 5. Individual data table (NIST) for cadmium in foods and dietary supplements...25

Table 6. Data summary table for cadmium in foods and dietary supplements. ...............26

Figure 10. Cadmium in SRM 3233 Fortified Breakfast Cereal (method comparison data summary view - digestion method)...................................................................27

Figure 11. Cadmium in candidate SRM 3532 Calcium Dietary Supplement (method comparison data summary view - digestion method)..............................................28

Figure 12. Cadmium in 3233 Fortified Breakfast Cereal (method comparison data summary view - instrumental method)................................................................29

Figure 13. Cadmium in candidate SRM 3532 Calcium Dietary Supplement (method

Figure 14. Cadmium in candidate SRM 3532 Calcium Dietary Supplement and SRM 3233 Fortified Breakfast Cereal (sample/control comparison view)......................31

\section{VITAMIN B IN FOODS $_{5}$}

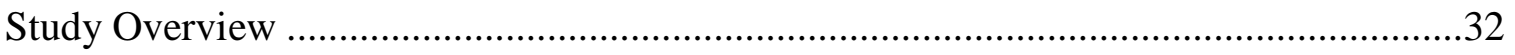

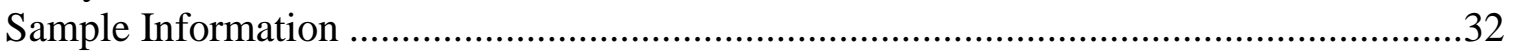

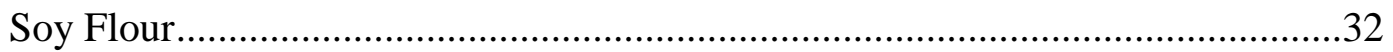

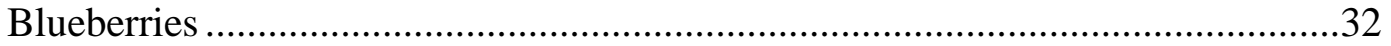

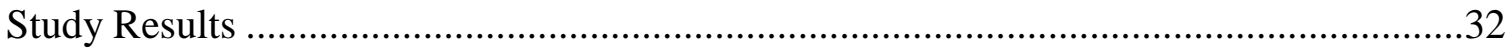

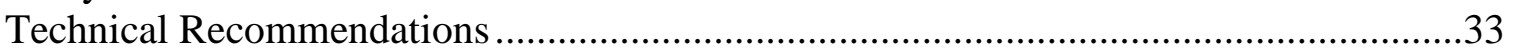

Table 7. Individual data table (NIST) for vitamin B5 (pantothenic acid) in foods...........34

Table 8. Data summary table for vitamin B5 (pantothenic acid) in foods........................35

Figure 15. Vitamin B5 (pantothenic acid) in SRM 3234 Soy Flour (data summary view)36

Figure 16. Vitamin B5 (pantothenic acid) in SRM 3287 Blueberries (data summary

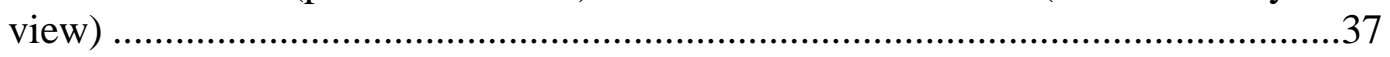

Figure 17. Vitamin B5 (pantothenic acid) in SRM 3234 Soy Flour and SRM 3287 Blueberries (sample/sample comparison view) ....................................................38

\section{VITAMIN A IN FOODS}

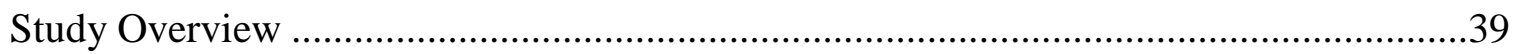

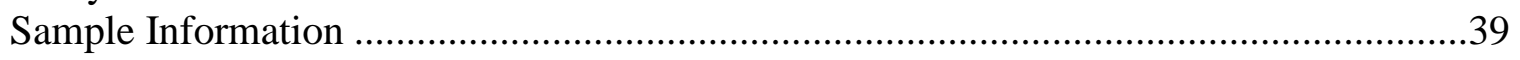

Multivitamin/Multielement Tablets ..................................................................39

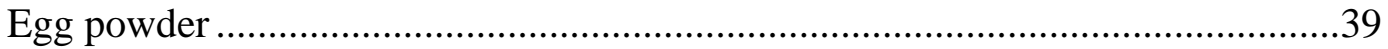

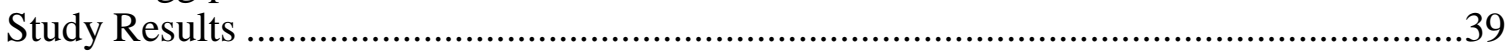

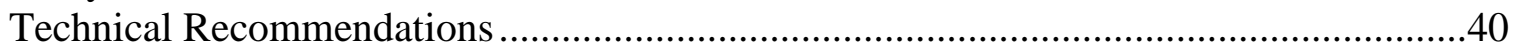

Table 9. Individual data table (NIST) for vitamin A in foods...........................................41

Table 10. Data summary table for retinol in foods. ........................................................42

Table 11. Data summary table for retinyl acetate in foods ...............................................43

Table 12. Data summary table for retinyl palmitate in foods.............................................44

Figure 18. Retinol in SRM 3280 Multivitamin/Multielement Tablet (data summary

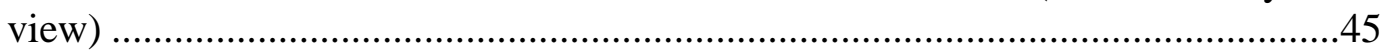

Figure 19. Retinol in whole egg powder (data summary view) .......................................46

Figure 20. Retinyl acetate in SRM 3280 Multivitamin/Multielement Tablets (data 
summary view)

Figure 21. Retinol in whole egg powder and SRM 3280 Multivitamin/Multielement Tablets (sample/control comparison view)

\section{CATECHINS IN GREEN TEA}

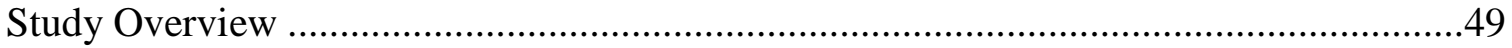

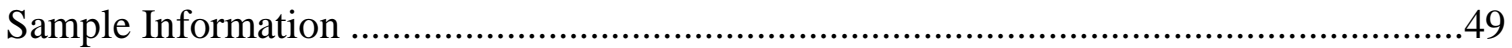

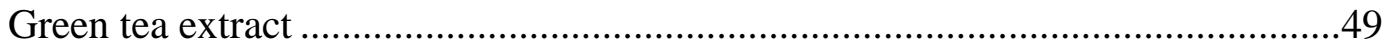

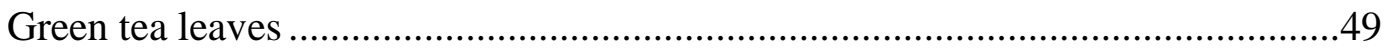

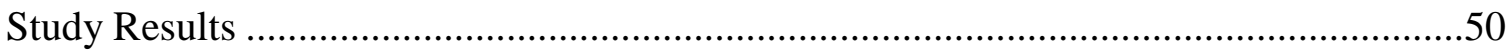

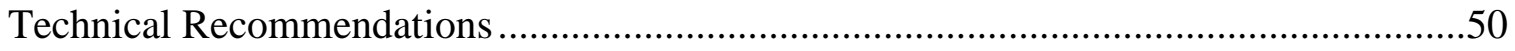

Table 13. Individual data table (NIST) for catechins in green tea ................................51

Table 14. Data summary table for catechin in green tea ............................................52

Table 15. Data summary table for epicatechin in green tea .........................................53

Table 16. Data summary table for epicatechin gallate in green tea ...............................54

Table 17. Data summary table for epigallocatechin in green tea .................................55

Table 18. Data summary table for epigallocatechin gallate in green tea .......................56

Table 19. Data summary table for gallocatechin in green tea.......................................57

Table 20. Data summary table for gallocatechin gallate in green tea ...........................58

Table 21. Data summary table for total catechins in green tea ....................................59

Figure 22. Catechin in SRM 3255 Camellia sinensis (Green Tea) Extract (data summary

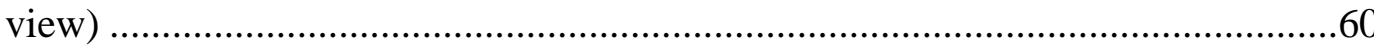

Figure 23. Catechin in SRM 3254 Camellia sinensis (Green Tea) (data summary view)61

Figure 24. Epicatechin in SRM 3255 Camellia sinensis (Green Tea) Extract (data

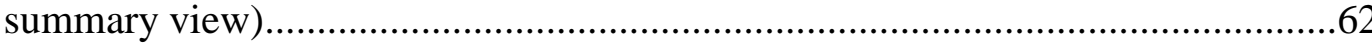

Figure 25. Epicatechin in SRM 3254 Camellia sinensis (Green Tea) (data summary

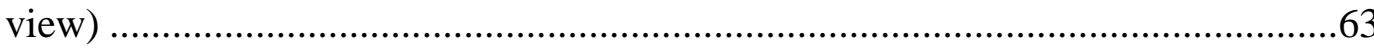

Figure 26. Epicatechin gallate in SRM 3255 Camellia sinensis (Green Tea) Extract (data

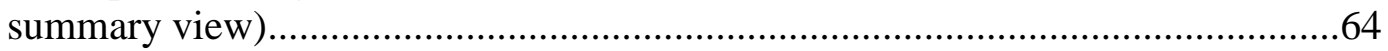

Figure 27. Epicatechin gallate in SRM 3254 Camellia sinensis (Green Tea) (data

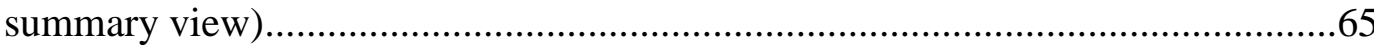

Figure 28. Epigallocatechin in SRM 3255 Camellia sinensis (Green Tea) Extract (data summary view) ........................................................................................66

Figure 29. Epigallocatechin in SRM 3254 Camellia sinensis (Green Tea) (data summary view)

Figure 30. Epigallocatechin gallate in SRM 3255 Camellia sinensis (Green Tea) Extract (data summary view)..............................................................................68

Figure 31. Epigallocatechin gallate in SRM 3254 Camellia sinensis (Green Tea) (data

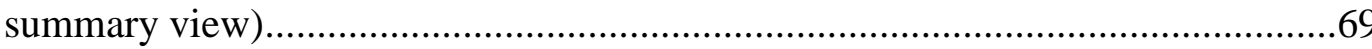

Figure 32. Gallocatechin in SRM 3255 Camellia sinensis (Green Tea) Extract (data summary view) ..................................................................................70

Figure 33. Gallocatechin in SRM 3254 Camellia sinensis (Green Tea) (data summary

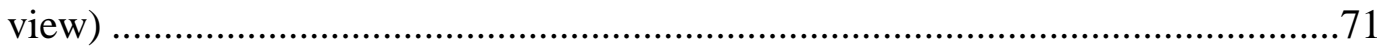

Figure 34. Gallocatechin gallate in SRM 3255 Camellia sinensis (Green Tea) Extract

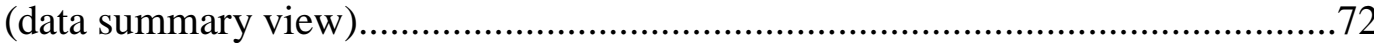

Figure 35. Gallocatechin gallate in SRM 3254 Camellia sinensis (Green Tea) (data 
summary view)

Figure 36. Total catechins in SRM 3255 Camellia sinensis (Green Tea) Extract (data summary view)

Figure 37. Total catechins in SRM 3254 Camellia sinensis (Green Tea) (data summary view) .75

Figure 38. Catechin in SRM 3254 Camellia sinensis (Green Tea) Leaves and SRM 3255 Camellia sinensis (Green Tea) Extract (sample/control comparison view) ...........76

Figure 39. Epicatechin in SRM 3254 Camellia sinensis (Green Tea) Leaves and SRM 3255 Camellia sinensis (Green Tea) Extract (sample/control comparison view) .77

Figure 40. Epicatechin gallate in SRM 3254 Camellia sinensis (Green Tea) Leaves and SRM 3255 Camellia sinensis (Green Tea) Extract (sample/control comparison view)

Figure 41. Epigallocatechin in SRM 3254 Camellia sinensis (Green Tea) Leaves and SRM 3255 Camellia sinensis (Green Tea) Extract (sample/control comparison view)

Figure 42. Epigallocatechin gallate in SRM 3254 Camellia sinensis (Green Tea) Leaves and SRM 3255 Camellia sinensis (Green Tea) Extract (sample/control comparison view)

Figure 43. Gallocatechin in SRM 3254 Camellia sinensis (Green Tea) Leaves and SRM 3255 Camellia sinensis (Green Tea) Extract (sample/control comparison view) .81

Figure 44. Gallocatechin gallate in SRM 3254 Camellia sinensis (Green Tea) Leaves and SRM 3255 Camellia sinensis (Green Tea) Extract (sample/control comparison view)

Figure 45. Total catechins in SRM 3254 Camellia sinensis (Green Tea) Leaves and SRM 3255 Camellia sinensis (Green Tea) Extract (sample/control comparison view) 


\section{ABSTRACT}

The NIST Dietary Supplement Laboratory Quality Assurance Program (DSQAP) was established in collaboration with the National Institutes of Health (NIH) Office of Dietary Supplements (ODS) in 2007 to enable members of the dietary supplements community to improve the accuracy of measurements for demonstration of compliance with various regulations. Exercise I of this program offered the opportunity for laboratories to assess their inhouse measurements of nutritional elements (Cr, Mo, and Se), contaminants (Cd), water-soluble vitamins (pantothenic acid), fat-soluble vitamins (retinol), and catechins in foods and/or botanical dietary supplement ingredients and finished products.

\section{INTRODUCTION}

The dietary supplement industry in the US is booming, with two-thirds of adults considering themselves to be supplement users. ${ }^{1}$ Consumption of dietary supplements, which includes vitamin and mineral supplements, represents an annual US expenditure of more than $\$ 25$ billion. These figures represent an increasing American trend, and as a result, it is critically important that both the quality and safety of these products are verified and maintained.

The Dietary Supplement Health and Education Act of 1994 (DSHEA) amended the Food, Drug and Cosmetic Act to create the regulatory category called dietary supplements. The DSHEA also gave the FDA authority to write current Good Manufacturing Practices (cGMPs) that require manufacturers to evaluate the identity, purity, and composition of their ingredients and finished products. To enable members of the dietary supplements community to improve the accuracy of the measurements required for compliance with these and other regulations, NIST established the Dietary Supplement Laboratory Quality Assurance Program (DSQAP) in collaboration with the NIH ODS in 2007.

The program offers the opportunity for laboratories to assess their in-house measurements of active or marker compounds, nutritional elements, contaminants (toxic elements, pesticides, mycotoxins), and fat- and water-soluble vitamins in foods as well as in botanical dietary supplement ingredients and finished products. Reports and certificates of participation are provided and can be used to demonstrate compliance with the cGMPs. In addition, NIST and the DSQAP assist the ODS Analytical Methods and Reference Materials program (AMRM) at the NIH in supporting the development and dissemination of analytical tools and reference materials. In the future, results from DSQAP exercises could be used by ODS to identify problematic matrices and analytes for which an Official Method of Analysis would benefit the dietary supplement community.

NIST has experience in the area of quality assurance programs, but the DSQAP takes a unique approach. In other NIST quality assurance programs, a set of analytes is measured repeatedly over time in the same or similar matrices to demonstrate laboratory performance. In contrast, the wide range of matrices and analytes under the "dietary supplement" umbrella means that not

\footnotetext{
${ }^{1}$ Walsh, T. (2012) Supplement Usage, Consumer Confidence Remain Steady According to New Annual Survey from CRN. Council for Responsible Nutrition, Washington, DC.
} 
every laboratory is interested in every sample or analyte. The constantly changing dietary supplement market, and the enormous diversity of finished products, makes repeated determination of a few target compounds in a single matrix of little use to participants. Instead, participating laboratories are interested in testing in-house methods on a wide variety of challenging, real-world matrices to demonstrate that their performance is comparable to that of the community and that their methods provide accurate results. In an area where there are few standard methods, the DSQAP offers a unique tool for assessment of the quality of measurements, provides feedback about performance, and can assist participants in improving laboratory operations.

This report summarizes the results from the ninth exercise of the DSQAP, Exercise I. Eightyfive laboratories responded to the call for participants distributed in October 2012. Samples were shipped to participants in December 2012, and results were returned to NIST by March 2013. This report contains the final data and information to be disseminated to the participants in July 2013.

\section{OVERVIEW OF DATA TREATMENT AND REPRESENTATION}

\section{$\underline{\text { Statistics }}$}

The individual data table and graphs contain information about the performance of each laboratory relative to that of the other participants in this study and relative to a target around the expected result (if available). The consensus mean and standard deviation are calculated according to the robust algorithm outlined in ISO 13528:2005(E), Annex C. ${ }^{2}$ The algorithm is summarized here in simplified form.

Initial values of the consensus mean, $x^{*}$, and consensus standard deviation, $s^{*}$, are estimated as

$$
\begin{array}{ll}
x^{*}=\text { median of } x_{i} & (i=1,2, \ldots, n) \\
s^{*}=1.483 \times \text { median of }\left|x_{i}-x^{*}\right| & (i=1,2, \ldots, n) .
\end{array}
$$

These initial values for $x^{*}$ and $s^{*}$ are updated by first calculating the expanded standard deviation, $\delta$, as

$$
\delta=1.5 \times s^{*} .
$$

Then each $x_{i}$ is compared to the expanded range and adjusted to $x_{i}{ }^{*}$ as described below to reduce the effect of outliers.

$$
\begin{aligned}
& \text { If } x_{i}<x^{*}-\delta \text {, then } x_{i}^{*}=x^{*}-\delta \text {. } \\
& \text { If } x_{i}>x^{*}+\delta \text {, then } x_{i}^{*}=x^{*}+\delta \text {. } \\
& \text { Otherwise, } x_{i}^{*}=x_{i} \text {. }
\end{aligned}
$$

New values of $x^{*}, s^{*}$, and $\delta$ are calculated iteratively until the process converges. Convergence is taken as no change from one iteration to the next in the third significant figure of $s^{*}$ and in the equivalent digit in $x^{*}$ : 


$$
\begin{aligned}
& x^{*}=\frac{\sum_{i=1}^{n} x_{i}^{*}}{n} \\
& s^{*}=1.134 \times \sqrt{\frac{\sum_{i=1}^{n}\left(x_{i}^{*}-x^{*}\right)}{n-1} .} .
\end{aligned}
$$

Individual Data Table

The data in this table is individualized to each participating laboratory and is provided to allow participants to directly compare their data to the summary statistics (consensus or community data as well as NIST certified, reference, or estimated values). The upper left of the data table includes the randomized laboratory code. Tables included in this report are generated using NIST data to protect the identity and performance of participants.

Section 1 of the data table contains the laboratory results as reported, including the mean and standard deviation when multiple values were reported. A blank indicates that NIST does not have data on file for that laboratory for a particular analyte or matrix. An empty box for standard deviation indicates that only a single value was reported and therefore that value was not included in the calculation of the consensus data. ${ }^{2}$

Also in Section 1 are two Z-scores. The first Z-score, $Z_{\text {comm }}$, is calculated with respect to the community consensus value, using $\mathrm{x}^{*}$ and $\mathrm{s} *$ :

$$
Z_{\text {comm }}=\frac{x_{i}-x *}{s^{*}} \text {. }
$$

The second Z-score, $\mathrm{Z}_{\mathrm{NIST}}$, is calculated with respect to the target value (NIST certified, reference, or estimated value), using $\mathrm{x}_{\mathrm{NIST}}$ and $U_{95}$ (the expanded uncertainty) or $\mathrm{s}_{\mathrm{NIST}}$, the standard deviation of NIST measurements:

$$
Z_{N I S T}=\frac{x_{i}-x_{N I S T}}{U_{95}}
$$

or

$$
Z_{N I S T}=\frac{x_{i}-x_{N I S T}}{s_{N I S T}}
$$

The significance of the Z-score is as follows:

- $|\mathrm{Z}|<2$ indicates that the laboratory result is considered to be within the community consensus range (for $\mathrm{Z}_{\mathrm{comm}}$ ) or NIST target range (for $\mathrm{Z}_{\mathrm{NIST}}$ ).

- $2<|\mathrm{Z}|<3$ indicates that the laboratory result is considered to be marginally different from the community consensus value (for $\mathrm{Z}_{\text {comm }}$ ) or NIST target value (for $\mathrm{Z}_{\mathrm{NIST}}$ ).

- $|Z|>3$ indicates that the laboratory result is considered to be significantly different from the community consensus value (for $\mathrm{Z}_{\text {comm }}$ ) or NIST target value (for $\mathrm{Z}_{\mathrm{NIST}}$ ).

\footnotetext{
${ }^{2}$ ISO 13528:2005(E), Statistical methods for use in proficiency testing by interlaboratory comparisons, pp 14-15.
} 
Section 2 of the data table contains the community results, including the number of laboratories reporting more than a single value for a given analyte ${ }^{1}$, the mean value determined for each analyte, and a robust estimate of the standard deviation of the reported values. ${ }^{3}$ Consensus means and standard deviations are calculated using the laboratory means; if a laboratory reported a single value, the reported value is not included. ${ }^{1}$ Additional information on calculation of the consensus mean and standard deviation can be found in the previous section.

Section 3 of the data table contains the target values for each analyte. When possible, the target value is a certified or reference value determined at NIST. Certified values and the associated expanded uncertainty $\left(U_{95}\right)$ have been determined with two independent analytical methods at NIST, by collaborating laboratories, or in some combination. Reference values are assigned using NIST values obtained from the average and standard deviation of measurements made using a single analytical method. For both certified and reference values, at least six samples have been tested and duplicate preparations from the sample package have been included, allowing the uncertainty to encompass variability due to inhomogeneity within and between packages. For samples in which a NIST certified or reference value is not available, the analytes are measured at NIST using an appropriate method. The NIST-assessed value represents the mean of at least three replicates. For materials acquired from another proficiency testing program, the consensus value and uncertainty from the completed round is used as the target range.

\section{Summary Data Table}

This data table includes a summary of all reported data for a particular analyte in a particular study. Participants can compare the raw data for a single laboratory to data reported by the other participating laboratories or to the consensus data. A blank indicates that the laboratory signed up and received samples for that particular analyte and matrix, but NIST does not have data on file for that laboratory.

\section{Graphs}

\section{Data Summary View (Method Comparison Data Summary View)}

In this view, individual laboratory data are plotted with the individual laboratory standard deviation (error bars). Data points that are unfilled represent laboratories that only reported a single value for that analyte and therefore were not included in the consensus mean. The black solid line represents the consensus mean, and the black dotted lines represent the consensus variability calculated as one standard deviation about the consensus mean. The gray shaded region represents the target zone for "acceptable" performance, which encompasses the NIST certified, reference, or estimated value bounded by twice its uncertainty $\left(U_{95}\right)$ or standard deviation. For the purpose of the DSQAP, a target range spanning twice the uncertainty in the NIST value is selected because participants are only asked to make a limited number of observations. The size of the y-axis on the data summary view graph represents the consensus mean bounded by $2 \delta$. In this view, the relative locations of individual laboratory data and consensus zones with respect to the target zone can be compared easily. In most cases, the target zone and the consensus zone overlap, which is the expected result. One program goal is to reduce the size of the consensus zone and center the consensus zone about the target value.

\footnotetext{
${ }^{3}$ ISO 13528:2005(E), Statistical methods for use in proficiency testing by interlaboratory comparisons, Annex C.
} 
Analysis of an appropriate reference material as part of a quality control scheme can help to identify sources of bias for laboratories reporting results that are significantly different from the target zone. In the case in which a method comparison is relevant, different colored data points may be used to indicate laboratories that used a specific approach to sample preparation, analytical method, or quantitation.

\section{Sample/Control Comparison View (Sample/Sample Comparison View)}

In this view, the individual laboratory results for a control (NIST SRM with a certified value) are compared to the results for an unknown (another NIST SRM with a more challenging matrix, a commercial sample, etc.). The error bars represent the individual laboratory standard deviation. The solid red box represents the target zone for the control (x-axis) and unknown sample ( $\mathrm{y}$ axis). The dotted blue box represents the consensus zone for the control (x-axis) and the unknown sample (y-axis). This view emphasizes trends in the data that may indicate potential calibration issues or method biases. One program goal is to identify such calibration or method biases and assist participants in improving analytical measurement capabilities. In some cases, when two equally challenging materials are provided, the same view (sample/sample comparison) can be helpful in identifying commonalities or differences in the analysis of the two materials. 


\section{TRACE NUTRITIONAL ELEMENTS IN FOODS AND SUPPLEMENTS}

\section{Study Overview}

In this study, participants were provided with one NIST SRM, SRM 3280

Multivitamin/Multielement Tablets, and a powdered whole egg material. Participants were asked to use in-house analytical methods to determine the mass fractions of three nutritional elements (chromium, molybdenum, and selenium) in each of the matrices and report values on an as-received basis.

\section{$\underline{\text { Sample Information }}$}

Multivitamin/multielement tablets. Participants were provided with one packet containing 15 multivitamin/multielement tablets. The material was produced by blending a vitamin and mineral pre-mix with a direct-compression tablet formulation. Intact tablets were heat-sealed inside $0.1 \mathrm{~mm}$ ( $4 \mathrm{mil}$ ) polyethylene bags, which were then sealed inside Mylar bags. Before use, participants were instructed to grind all tablets together, mix the resulting powder thoroughly, and use a sample size of at least $0.5 \mathrm{~g}$. Participants were asked to store the material at controlled room temperature, $10^{\circ} \mathrm{C}$ to $30^{\circ} \mathrm{C}$, prepare three samples, and report three values from the resulting ground material. Approximate analyte levels were not provided to participants prior to the study. NIST certified values in SRM 3280 were determined using inductively coupled plasma mass spectrometry (ICP-MS), inductively coupled plasma optical emission spectrometry (ICP-OES), instrumental neutron activation analysis (INAA), and X-ray florescence spectroscopy (XRF). The certified values and uncertainties for Cr, Mo, and Se in SRM 3280 are outlined in the table below, both on a dry-mass basis and an as-received basis following adjustment for the moisture content of the material $(1.37 \%)$.

\begin{tabular}{ccccc} 
Analyte & $\begin{array}{c}\text { Certified Mass Fraction }(\mathrm{mg} / \mathrm{kg}) \\
\text { (dry-mass basis) }\end{array}$ & $\begin{array}{c}\text { Adjusted Mass Fraction }(\mu \mathrm{g} / \mathrm{g}) \\
\text { (as-received basis) }\end{array}$ \\
\cline { 1 - 3 } & $93.7 \pm 2.7$ & 92.4 & \pm & 2.7 \\
Mo & $70.7 \pm 4.5$ & 69.7 & \pm & 4.4 \\
Se & $17.42 \pm 0.45$ & $17.2 \pm \pm$ & \pm .4
\end{tabular}

Whole egg powder. Participants were provided with one packet containing approximately $10 \mathrm{~g}$ of commercially available whole egg powder. The whole egg powder is a free-flowing, fine powder prepared from USDA-inspected eggs. The powder was heat-sealed inside nitrogenflushed $0.1 \mathrm{~mm}$ (4 mil) polyethylene bags, which were then sealed inside aluminized plastic bags. Before use, participants were instructed to thoroughly mix the contents of the packet and use a sample size of at least $0.5 \mathrm{~g}$. Participants were asked to store the material at controlled room temperature, $10^{\circ} \mathrm{C}$ to $30{ }^{\circ} \mathrm{C}$ and report three values from the single packet provided. Approximate analyte levels were not provided prior to the study. NIST reported values for Cr, Mo, and Se using microwave digestion and inductively coupled plasma mass spectrometry (ICPMS) with standard additions as the method of quantitation. The NIST values in whole egg powder are reported in the table below with an estimated relative uncertainty of $5 \%$. 


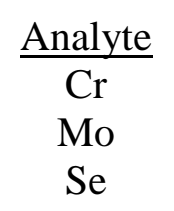

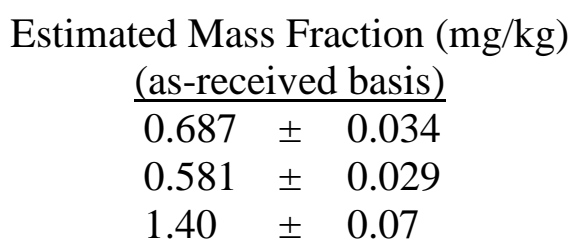

Study Results

- Fifty-three laboratories enrolled in this exercise and received samples with a minimum of 37 laboratories reporting results for one or more elements (70\% participation).

- The consensus means for chromium and molybdenum in the multivitamin/multielement tablets were within the target range with an acceptable variability (14\% and $16 \%$ relative standard deviation (RSD), respectively). The consensus mean for selenium in the multivitamin/multielement tablets was below the target range, with a slightly higher variability (19\% RSD).

- The consensus means for molybdenum and selenium in the whole egg powder were within the target range. While molybdenum had an acceptable variability (14 \% RSD), the variability for selenium was higher (26 \% RSD). The consensus mean for chromium in the whole egg powder was above the target range with an unacceptable variability of $63 \%$ RSD.

- A majority of the laboratories reported using either open-beaker digestion (29\% to $36 \%$, depending on the element) or microwave digestion (52\% to $58 \%$ ) for sample preparation. The remaining laboratories reported using hot block digestion (11\% to 13 $\%)$.

- A majority of the laboratories reported using either ICP-MS (72\% to $81 \%$, depending on the element) or ICP-OES (18\% to $23 \%$ ) as their analytical method. Less than $5 \%$ of the laboratories reported using atomic absorption spectroscopy (AAS) or total reflection Xray fluorescence (TXRF).

\section{Technical Recommendations}

The following recommendations are based on results reported by the participants in this study.

- There did not seem to be a difference in results based on either open-beaker digestions or microwave digestions for the elements in this study. There also did not appear to be any difference in results based on either ICP-OES or ICP-MS analytical methods. (Too few results were reported by other methods to identify any trends).

- Laboratories that reported high values for one material and low values for the second material for any particular element (see Figure 7, Figure 8, and Figure 9) may have more trouble digesting one sample matrix over the other. SRM 3280 Multivitamin/Multielement Tablets are very difficult to digest, requiring relatively high temperatures, regardless of digestion method, to get complete sample dissolution. Laboratories using higher temperatures for digestions were more consistent at reporting values within consensus or target ranges for all elements.

- It is important to note that with different sample matrices, there may also be different interferences to take into consideration during sample analysis.

- The elongated consensus box in Figure 7 is due to several high values reported for the whole egg powder. There are several possibilities for this, one being calibration errors. 
- The concentrations of these three elements in whole egg powder was approximately 10 to 100 times less than those in SRM 3280

Multivitamin/Multielement Tablets so there was the possibility of contamination if the two materials were prepared together.

- With both ICP-OES and ICP-MS, it is important to check the calibration curve for linearity within the range of the sample solutions.

- With ICP-OES, some elements will not be linear beyond an upper limit. Make sure solution concentrations fall within that linear range.

- With ICP-MS, many instruments run in pulse mode, which is more sensitive. If the calibration curve extends beyond the dynamic range for pulse mode then the instrument will use both the pulse and analog mode. The ICP-MS must be calibrated for both modes in this case. It is often easier and more accurate to have a narrower range of calibration points, making sure the calibration curve is linear in the pulse mode.

- Run a quality control sample of known accuracy to ensure your method is performing as expected.

- Double-check all calculations for any errors. 
Table 1. Individual data table (NIST) for trace nutritional elements in foods and dietary supplements.

\section{National Institute of Standards \& Technology}

Exercise I - October 2012 - Nutritional Elements

\begin{tabular}{ccc} 
& Lab Code: & NIST \\
\hline Analyte & Sample & Units \\
\hline Cr & Multivitamin Tablet & $\mu \mathrm{g} / \mathrm{g}$ \\
$\mathrm{Cr}$ & Egg Powder & $\mu \mathrm{g} / \mathrm{g}$ \\
\hline Mo & Multivitamin Tablet & $\mu \mathrm{g} / \mathrm{g}$ \\
Mo & Egg Powder & $\mu \mathrm{g} / \mathrm{g}$ \\
\hline Se & Multivitamin Tablet & $\mu \mathrm{g} / \mathrm{g}$ \\
Se & Egg Powder & $\mu \mathrm{g} / \mathrm{g}$ \\
\hline
\end{tabular}

\begin{tabular}{cccc}
\multicolumn{4}{c}{ 1. Your Results } \\
\hline $\mathrm{x}_{\mathrm{i}}$ & $\mathrm{s}_{\mathrm{i}}$ & $\mathrm{Z}_{\text {comm }}$ & $\mathrm{Z}_{\mathrm{NIST}}$ \\
\hline 92.4 & 2.7 & 0.2 & 0.0 \\
0.687 & 0.034 & -0.2 & 0.0 \\
\hline 69.7 & 4.4 & -0.1 & 0.0 \\
0.581 & 0.029 & 0.0 & 0.0 \\
\hline 17.2 & 0.4 & 0.4 & 0.0 \\
1.40 & 0.07 & -0.1 & 0.0 \\
\hline
\end{tabular}

\begin{tabular}{ccc}
\multicolumn{3}{c}{ 2. Community Results } \\
\hline $\mathrm{N}$ & $\mathrm{x} *$ & $\mathrm{~s}^{*}$ \\
\hline 41 & 89.8 & 12.7 \\
38 & 0.808 & 0.511 \\
\hline 39 & 70.4 & 11.2 \\
35 & 0.580 & 0.083 \\
\hline 39 & 16.1 & 3.0 \\
36 & 1.44 & 0.38 \\
\hline
\end{tabular}

\begin{tabular}{cc}
\multicolumn{2}{c}{ 3. Target } \\
\hline $\mathrm{X}_{\mathrm{NIST}}$ & $U_{95}$ \\
\hline 92.4 & 2.7 \\
0.687 & 0.034 \\
\hline 69.7 & 4.4 \\
0.581 & 0.029 \\
\hline 17.2 & 0.4 \\
1.40 & 0.07 \\
\hline
\end{tabular}

$\mathrm{x}_{\mathrm{i}} \quad$ Mean of reported values

$\mathrm{N}$ Number of quantitative

$\mathrm{x}_{\mathrm{NIST}}$ values reported

$\mathrm{s}_{\mathrm{i}} \quad$ Standard deviation of reported values

$Z_{\text {comm }} \quad$ Z-score with respect to community

x* Robust mean of reported values

NIST-assessed value Consensus

s* Robust standard deviation

$U_{95}$

$\pm 95 \%$ confidence interval about the assessed value or standard deviation ( $\mathrm{s}_{\mathrm{NIST}}$ ) 
Table 2. Data summary table for chromium in foods and dietary supplements.

\begin{tabular}{|c|c|c|c|c|c|c|c|c|c|c|c|}
\hline & \multirow[b]{3}{*}{ Lab } & \multicolumn{10}{|c|}{ Chromium } \\
\hline & & \multicolumn{5}{|c|}{ SRM 3280 Multivitamin Tablet $(\mu \mathrm{g} / \mathrm{g})$} & \multicolumn{5}{|c|}{ Whole Egg Powder ( $\mu g / g)$} \\
\hline & & $\mathbf{A}$ & B & $\mathbf{C}$ & Avg & SD & A & B & C & Avg & SD \\
\hline \multirow{54}{*}{ 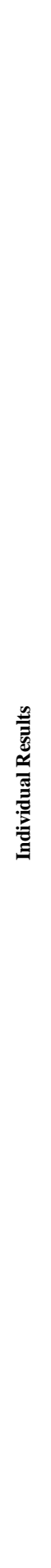 } & NIST & & & & 92.4 & 2.7 & & & & 0.687 & 0.034 \\
\hline & I901 & 70.3 & 65.1 & 67.6 & 67.7 & 2.6 & 0.610 & 0.560 & 0.640 & 0.603 & 0.040 \\
\hline & I903 & 99.5 & 97.6 & 98.2 & 98.4 & 1.0 & 0.536 & 0.527 & 0.528 & 0.530 & 0.005 \\
\hline & I904 & & & & & & & & & & \\
\hline & I906 & & & & & & & & & & \\
\hline & I907 & & & & & & & & & & \\
\hline & I908 & 92.5 & 89.2 & 88.7 & 90.1 & 2.1 & 0.451 & 0.444 & 0.454 & 0.450 & 0.005 \\
\hline & I910 & 92.4 & 101.0 & 97.9 & 97.1 & 4.4 & 1.060 & 1.190 & 1.220 & 1.157 & 0.085 \\
\hline & I911 & 89.9 & 90.4 & 93.7 & 91.3 & 2.1 & 0.450 & 0.410 & 0.440 & 0.433 & 0.021 \\
\hline & I915 & & & & & & & & & & \\
\hline & I917 & 89.5 & 88.2 & 89.7 & 89.1 & 0.8 & 1.318 & 1.368 & 1.414 & 1.367 & 0.048 \\
\hline & I920 & 111.5 & 110.8 & 113.0 & 111.8 & 1.1 & 0.660 & 0.656 & 0.650 & 0.655 & 0.005 \\
\hline & I925 & 77.3 & 84.0 & 92.6 & 84.7 & 7.7 & 1.280 & 1.372 & 1.789 & 1.480 & 0.271 \\
\hline & I928 & 82.1 & 76.8 & 80.8 & 79.9 & 2.8 & 1.200 & 1.100 & 1.200 & 1.167 & 0.058 \\
\hline & I930 & 95.9 & 108.8 & 107.3 & 104.0 & 7.0 & 0.518 & 0.522 & 0.675 & 0.572 & 0.090 \\
\hline & I931 & 77.7 & 89.5 & 88.1 & 85.1 & 6.4 & 0.631 & 0.553 & 0.520 & 0.568 & 0.057 \\
\hline & I932 & 79.3 & 84.7 & 85.1 & 83.0 & 3.2 & 0.450 & 0.450 & 0.469 & 0.456 & 0.011 \\
\hline & I933 & 98.0 & 99.0 & 101.0 & 99.3 & 1.5 & & & & & \\
\hline & I934 & 89.5 & 84.0 & 92.7 & 88.7 & 4.4 & 8.873 & 9.132 & 3.049 & 7.018 & 3.439 \\
\hline & I935 & & & & & & & & & & \\
\hline & I936 & 79.0 & 77.0 & 82.0 & 79.3 & 2.5 & 0.430 & 0.390 & 0.510 & 0.443 & 0.061 \\
\hline & I938 & & & & & & & & & & \\
\hline & I939 & 72.8 & & & 72.8 & & 0.801 & & & 0.801 & \\
\hline & I940 & 95.3 & 102.4 & 86.9 & 94.9 & 7.8 & 1.393 & 0.557 & 0.659 & 0.869 & 0.456 \\
\hline & I941 & 93.2 & 92.7 & 86.2 & 90.7 & 3.9 & 0.418 & 0.315 & 0.279 & 0.337 & 0.072 \\
\hline & I942 & 80.0 & 84.0 & 84.0 & 82.7 & 2.3 & 1.200 & 1.200 & 1.200 & 1.200 & 0.000 \\
\hline & I943 & 67.4 & 65.0 & 70.9 & 67.8 & 3.0 & 0.360 & 0.350 & 0.340 & 0.350 & 0.010 \\
\hline & I944 & & & & & & & & & & \\
\hline & I947 & 87.4 & 108.9 & 112.9 & 103.0 & 13.7 & 0.432 & 0.463 & 0.474 & 0.456 & 0.022 \\
\hline & I948 & 81.4 & 86.6 & 91.9 & 86.6 & 5.3 & 0.400 & 0.400 & 0.400 & 0.400 & 0.000 \\
\hline & I949 & 84.8 & 84.9 & 80.5 & 83.4 & 2.5 & 1.800 & 2.000 & 2.600 & 2.133 & 0.416 \\
\hline & I950 & & & & & & & & & & \\
\hline & I951 & 93.0 & 88.8 & 95.1 & 92.3 & 3.2 & 0.434 & 0.417 & 0.409 & 0.420 & 0.013 \\
\hline & I953 & 89.3 & 121.8 & 103.5 & 104.9 & 16.3 & 0.638 & 0.631 & 0.683 & 0.651 & 0.028 \\
\hline & I954 & 93.9 & 94.9 & 87.0 & 91.9 & 4.3 & & & & & \\
\hline & I955 & 95.9 & 98.2 & 95.1 & 96.4 & 1.6 & 2.621 & 3.323 & 3.612 & 3.185 & 0.510 \\
\hline & I956 & 87.7 & 92.1 & 94.8 & 91.5 & 3.6 & 2.917 & 3.646 & 2.896 & 3.153 & 0.427 \\
\hline & I958 & 147.9 & 151.3 & 149.2 & 149.5 & 1.7 & 0.200 & 0.200 & 0.200 & 0.200 & 0.000 \\
\hline & I959 & 91.0 & 93.0 & 93.8 & 92.6 & 1.5 & 0.569 & 0.515 & 0.601 & 0.562 & 0.044 \\
\hline & I960 & 92.9 & 103.9 & & 98.4 & 7.8 & 0.461 & 0.461 & 0.425 & 0.449 & 0.021 \\
\hline & I961 & 84.1 & 84.3 & 80.0 & 82.8 & 2.4 & 0.807 & 0.775 & 0.745 & 0.776 & 0.031 \\
\hline & I963 & 98.1 & 92.2 & 91.9 & 94.1 & 3.5 & & & & & \\
\hline & I964 & 71.9 & 68.4 & 70.8 & 70.4 & 1.8 & 0.480 & 0.520 & 0.500 & 0.500 & 0.020 \\
\hline & I965 & & & & & & & & & & \\
\hline & I966 & 55.9 & 56.4 & 48.6 & 53.7 & 4.4 & 1.600 & 1.040 & 1.040 & 1.227 & 0.323 \\
\hline & I967 & 101.0 & 105.0 & 108.0 & 104.7 & 3.5 & 1.770 & 1.500 & 1.800 & 1.690 & 0.165 \\
\hline & I972 & 97.1 & 98.4 & 98.2 & 97.9 & 0.7 & 0.472 & 0.505 & 0.489 & 0.489 & 0.017 \\
\hline & I973 & 79.0 & 81.7 & 90.2 & 83.6 & 5.8 & 0.465 & 0.432 & 0.431 & 0.443 & 0.019 \\
\hline & I978 & 62.3 & 70.3 & 69.5 & 67.4 & 4.4 & 0.518 & 0.484 & 0.589 & 0.530 & 0.054 \\
\hline & I980 & 108.0 & 95.0 & 97.9 & 100.3 & 6.8 & 0.795 & 0.683 & 0.730 & 0.736 & 0.056 \\
\hline & I981 & 82.9 & 76.3 & 79.8 & 79.6 & 3.3 & 2.280 & 2.650 & 2.280 & 2.403 & 0.214 \\
\hline & I983 & 108.0 & 105.0 & & 106.5 & 2.1 & & & & & \\
\hline & I985 & 71.7 & & & 71.7 & & 0.780 & & & 0.780 & \\
\hline & I986 & & & & & & 0.673 & 0.714 & 0.674 & 0.687 & 0.023 \\
\hline \multirow{5}{*}{ 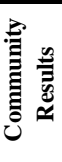 } & & \multicolumn{3}{|c|}{ Consensus Mean } & \multicolumn{2}{|l|}{89.8} & \multicolumn{3}{|c|}{ Consensus Mean } & 0.808 & \\
\hline & & \multicolumn{3}{|c|}{ Consensus Standard Deviation } & \multicolumn{2}{|l|}{12.7} & \multicolumn{3}{|c|}{ Consensus Standard Deviation } & 0.511 & \\
\hline & & Maximun & & & 149.5 & & Maximun & & & 7.018 & \\
\hline & & Minimum & & & 53.7 & & Minimum & & & 0.200 & \\
\hline & & $\mathrm{N}$ & & & 41 & & $\mathrm{~N}$ & & & 38 & \\
\hline
\end{tabular}


Table 3. Data summary table for molybdenum in foods and dietary supplements.

\begin{tabular}{|c|c|c|c|c|c|c|c|c|c|c|c|}
\hline & \multirow[b]{3}{*}{ Lab } & \multicolumn{10}{|c|}{ Molybdenum } \\
\hline & & \multicolumn{5}{|c|}{ SRM 3280 Multivitamin Tablet $(\mu \mathrm{g} / \mathrm{g})$} & \multicolumn{5}{|c|}{ Whole Egg Powder ( $\mu \mathrm{g} / \mathrm{g})$} \\
\hline & & A & B & C & Avg & SD & A & B & C & Avg & SD \\
\hline \multirow{54}{*}{ 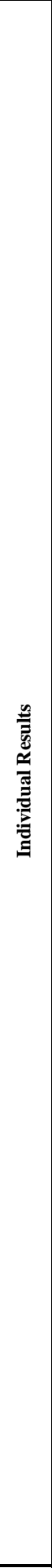 } & NIST & & & & 69.7 & 4.4 & & & & 0.581 & 0.029 \\
\hline & I901 & & & & & & & & & & \\
\hline & I903 & 71.3 & 68.7 & 66.3 & 68.8 & 2.5 & 0.615 & 0.598 & 0.608 & 0.607 & 0.009 \\
\hline & I904 & & & & & & & & & & \\
\hline & I906 & & & & & & & & & & \\
\hline & I907 & & & & & & & & & & \\
\hline & I908 & 86.5 & 71.5 & 84.4 & 80.8 & 8.1 & 0.716 & 0.495 & 0.440 & 0.550 & 0.146 \\
\hline & I910 & 65.0 & 62.5 & 70.2 & 65.9 & 3.9 & 1.090 & 0.632 & 0.511 & 0.744 & 0.305 \\
\hline & I911 & 66.7 & 65.6 & 63.2 & 65.2 & 1.8 & 0.556 & 0.522 & 0.554 & 0.544 & 0.019 \\
\hline & I915 & & & & & & & & & & \\
\hline & I917 & 72.8 & 72.3 & 70.6 & 71.9 & 1.1 & 0.581 & 0.582 & 0.591 & 0.585 & 0.006 \\
\hline & I920 & 76.7 & 62.1 & 69.3 & 69.3 & 7.3 & 0.606 & 0.622 & 0.600 & 0.609 & 0.011 \\
\hline & I925 & 64.9 & 69.9 & 74.5 & 69.8 & 4.8 & 1.386 & 1.352 & 1.756 & 1.498 & 0.224 \\
\hline & I928 & 89.1 & 81.7 & 83.8 & 84.9 & 3.8 & 0.500 & 0.500 & 0.500 & 0.500 & 0.000 \\
\hline & I930 & 55.4 & 58.2 & 56.1 & 56.6 & 1.4 & 0.531 & 0.523 & 0.492 & 0.515 & 0.021 \\
\hline & I931 & 78.7 & 67.6 & 75.6 & 74.0 & 5.7 & 0.578 & 0.570 & 0.575 & 0.574 & 0.004 \\
\hline & I932 & 94.2 & 108.4 & 108.7 & 103.8 & 8.3 & 0.640 & 0.640 & 0.633 & 0.638 & 0.004 \\
\hline & I933 & 70.0 & 74.0 & 72.0 & 72.0 & 2.0 & & & & & \\
\hline & I934 & 66.6 & 55.7 & 64.9 & 62.4 & 5.9 & 0.678 & 0.747 & 0.598 & 0.674 & 0.074 \\
\hline & I935 & & & & & & & & & & \\
\hline & I936 & 86.0 & 73.0 & 74.0 & 77.7 & 7.2 & 0.570 & 0.550 & 0.560 & 0.560 & 0.010 \\
\hline & I938 & & & & & & & & & & \\
\hline & I939 & 52.8 & & & 52.8 & & 0.361 & & & 0.361 & \\
\hline & I940 & 74.4 & 110.1 & 70.5 & 85.0 & 21.8 & 0.774 & 0.604 & 0.540 & 0.639 & 0.121 \\
\hline & I941 & 61.7 & 61.9 & 62.6 & 62.1 & 0.5 & 0.186 & 0.159 & 0.146 & 0.164 & 0.021 \\
\hline & I942 & 61.0 & 65.0 & 68.0 & 64.7 & 3.5 & 0.550 & 0.550 & 0.530 & 0.543 & 0.012 \\
\hline & I943 & 65.5 & 65.2 & 67.8 & 66.2 & 1.4 & 0.520 & 0.520 & 0.510 & 0.517 & 0.006 \\
\hline & I944 & & & & & & & & & & \\
\hline & I947 & 76.2 & 80.7 & 78.3 & 78.4 & 2.3 & 0.544 & 0.498 & 0.527 & 0.523 & 0.023 \\
\hline & I948 & 71.1 & 72.4 & 77.9 & 73.8 & 3.6 & 0.570 & 0.580 & 0.580 & 0.577 & 0.006 \\
\hline & I949 & 49.5 & 54.6 & 53.1 & 52.4 & 2.6 & 0.900 & 0.500 & 0.700 & 0.700 & 0.200 \\
\hline & I950 & & & & & & & & & & \\
\hline & I951 & 87.5 & 81.0 & 70.4 & 79.6 & 8.6 & 0.695 & 0.658 & 0.686 & 0.680 & 0.019 \\
\hline & I953 & 88.0 & 78.8 & 71.4 & 79.4 & 8.3 & 0.566 & 0.581 & 0.585 & 0.577 & 0.010 \\
\hline & I954 & 60.0 & 67.7 & 60.7 & 62.8 & 4.3 & & & & & \\
\hline & I955 & 72.0 & 65.6 & 72.7 & 70.1 & 3.9 & 0.449 & 0.453 & 0.447 & 0.450 & 0.003 \\
\hline & I956 & 67.0 & 90.7 & 56.0 & 71.2 & 17.8 & 0.467 & 0.472 & 0.459 & 0.466 & 0.007 \\
\hline & I958 & 87.5 & 84.8 & 82.2 & 84.8 & 2.7 & & & & & \\
\hline & I959 & 61.5 & 55.7 & 55.9 & 57.7 & 3.3 & 0.606 & 0.579 & 0.607 & 0.597 & 0.016 \\
\hline & I960 & 64.8 & 87.2 & & 76.0 & 15.8 & 0.514 & 0.548 & 0.488 & 0.517 & 0.030 \\
\hline & I961 & 63.0 & 62.2 & 15.0 & 46.7 & 27.5 & 0.798 & 0.776 & 0.366 & 0.647 & 0.244 \\
\hline & I963 & 76.3 & 79.8 & 76.7 & 77.6 & 1.9 & & & & & \\
\hline & I964 & 84.4 & 86.3 & 85.8 & 85.5 & 1.0 & 0.600 & 0.620 & 0.600 & 0.607 & 0.012 \\
\hline & I965 & & & & & & & & & & \\
\hline & I966 & & & & & & & & & & \\
\hline & I967 & 70.4 & 58.5 & 79.9 & 69.6 & 10.7 & 0.630 & 0.530 & 0.630 & 0.597 & 0.058 \\
\hline & I972 & 67.5 & 60.4 & 71.4 & 66.4 & 5.6 & 0.557 & 0.559 & 0.549 & 0.555 & 0.005 \\
\hline & I973 & 72.3 & 82.1 & 75.1 & 76.5 & 5.0 & 0.515 & 0.494 & 0.507 & 0.505 & 0.011 \\
\hline & I978 & 47.8 & 52.3 & 52.9 & 51.0 & 2.8 & 0.622 & 0.541 & 0.698 & 0.620 & 0.078 \\
\hline & I980 & 77.0 & 72.9 & 80.2 & 76.7 & 3.7 & 0.602 & 0.613 & 0.602 & 0.606 & 0.006 \\
\hline & I981 & 82.8 & 70.3 & 77.8 & 77.0 & 6.2 & 0.820 & 0.640 & 0.590 & 0.683 & 0.121 \\
\hline & I983 & 53.0 & 56.0 & & 54.5 & 2.1 & & & & & \\
\hline & I985 & 50.1 & & & 50.1 & & 0.460 & & & 0.460 & \\
\hline & I986 & & & & & & 0.592 & 0.581 & 0.571 & 0.581 & 0.011 \\
\hline \multirow{5}{*}{ 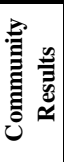 } & & \multicolumn{3}{|c|}{ Consensus Mean } & \multicolumn{2}{|l|}{70.4} & \multicolumn{3}{|c|}{ Consensus Mean } & 0.580 & \\
\hline & & \multicolumn{3}{|c|}{ Consensus Standard Deviation } & \multicolumn{2}{|l|}{11.2} & \multicolumn{3}{|c|}{ Consensus Standard Deviation } & 0.083 & \\
\hline & & Maximu & & & 104 & & Maximun & & & 1.498 & \\
\hline & & Minimur & & & 47 & & Minimum & & & 0.164 & \\
\hline & & $\mathrm{N}$ & & & 39 & & $\mathrm{~N}$ & & & 35 & \\
\hline
\end{tabular}


Table 4. Data summary table for selenium in foods and dietary supplements.

\begin{tabular}{|c|c|c|c|c|c|c|c|c|c|c|c|}
\hline & \multirow[b]{3}{*}{ Lab } & \multicolumn{10}{|c|}{ Selenium } \\
\hline & & \multicolumn{5}{|c|}{ SRM 3280 Multivitamin Tablet $(\mu \mathrm{g} / \mathrm{g})$} & \multicolumn{5}{|c|}{ Whole Egg Powder ( $\mu \mathrm{g} / \mathrm{g})$} \\
\hline & & A & B & C & Avg & SD & $\mathbf{A}$ & B & C & Avg & SD \\
\hline \multirow{54}{*}{ 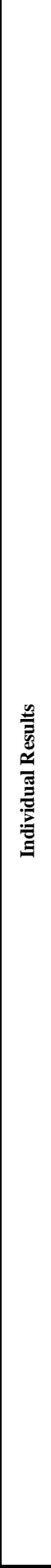 } & NIST & & & & 17.2 & 0.4 & & & & 1.40 & 0.07 \\
\hline & I901 & & & & & & & & & & \\
\hline & I903 & 16.4 & 15.7 & 17.0 & 16.4 & 0.7 & 1.39 & 1.40 & 1.42 & 1.40 & 0.02 \\
\hline & I904 & & & & & & & & & & \\
\hline & I906 & & & & & & & & & & \\
\hline & I907 & & & & & & & & & & \\
\hline & I908 & 18.4 & 17.9 & 16.0 & 17.4 & 1.3 & 1.65 & 1.59 & 1.57 & 1.60 & 0.04 \\
\hline & I910 & 17.7 & 18.0 & 16.2 & 17.3 & 1.0 & 0.93 & 1.50 & 1.13 & 1.19 & 0.29 \\
\hline & I911 & 17.0 & 14.8 & 15.6 & 15.8 & 1.1 & 1.60 & 1.50 & 1.50 & 1.53 & 0.06 \\
\hline & I915 & & & & & & & & & & \\
\hline & I917 & 14.9 & 15.1 & 14.6 & 14.9 & 0.2 & 2.14 & 2.20 & 2.06 & 2.13 & 0.07 \\
\hline & I920 & 12.7 & 11.6 & 11.2 & 11.8 & 0.8 & 1.45 & 1.49 & 1.44 & 1.46 & 0.03 \\
\hline & I925 & 17.8 & 17.7 & 17.5 & 17.7 & 0.1 & 1.40 & 1.37 & 1.46 & 1.41 & 0.05 \\
\hline & I928 & 24.9 & 20.6 & 22.7 & 22.7 & 2.2 & 1.80 & 1.90 & 1.90 & 1.87 & 0.06 \\
\hline & I930 & 14.0 & 14.9 & 13.8 & 14.2 & 0.6 & 1.67 & 1.53 & 1.49 & 1.56 & 0.09 \\
\hline & I931 & 16.2 & 15.4 & 15.7 & 15.7 & 0.4 & 1.74 & 1.57 & 1.68 & 1.66 & 0.09 \\
\hline & I932 & 11.7 & 12.2 & 13.6 & 12.5 & 1.0 & 1.58 & 1.60 & 1.48 & 1.55 & 0.07 \\
\hline & I933 & & & & & & & & & & \\
\hline & I934 & 18.7 & 17.5 & 18.1 & 18.1 & 0.6 & 1.43 & 0.95 & 0.94 & 1.11 & 0.28 \\
\hline & I935 & & & & & & & & & & \\
\hline & I936 & 20.0 & 18.0 & 19.0 & 19.0 & 1.0 & 1.50 & 1.70 & 1.60 & 1.60 & 0.10 \\
\hline & I938 & & & & & & & & & & \\
\hline & I939 & 8.8 & & & 8.8 & & 1.81 & & & 1.81 & \\
\hline & I940 & 11.0 & 13.2 & 12.2 & 12.1 & 1.1 & 0.97 & 0.09 & 0.97 & 0.68 & 0.51 \\
\hline & I941 & 14.9 & 15.1 & 15.2 & 15.0 & 0.1 & 0.73 & 0.76 & 0.76 & 0.75 & 0.02 \\
\hline & I942 & 15.0 & 16.0 & 17.0 & 16.0 & 1.0 & 1.70 & 1.70 & 1.60 & 1.67 & 0.06 \\
\hline & I943 & 18.6 & 15.9 & 16.4 & 17.0 & 1.4 & 1.36 & 1.40 & 1.20 & 1.32 & 0.11 \\
\hline & I944 & & & & & & & & & & \\
\hline & I947 & 23.1 & 22.5 & 20.7 & 22.1 & 1.3 & 1.47 & 1.37 & 1.49 & 1.44 & 0.06 \\
\hline & I948 & 16.3 & 15.5 & 17.8 & 16.5 & 1.2 & 1.40 & 1.40 & 1.40 & 1.40 & 0.00 \\
\hline & I949 & 15.3 & 15.3 & 14.5 & 15.0 & 0.5 & 1.20 & 1.00 & 1.30 & 1.17 & 0.15 \\
\hline & I950 & & & & & & & & & & \\
\hline & I951 & 19.3 & 15.6 & 17.5 & 17.4 & 1.9 & 1.44 & 1.35 & 1.39 & 1.39 & 0.05 \\
\hline & I953 & 6.5 & 9.3 & 6.4 & 7.4 & 1.6 & 0.83 & 0.83 & 0.82 & 0.83 & 0.01 \\
\hline & I954 & 16.2 & 17.1 & 15.4 & 16.2 & 0.9 & & & & & \\
\hline & I955 & 17.8 & 16.3 & 16.8 & 17.0 & 0.8 & 1.69 & 1.71 & 1.70 & 1.70 & 0.01 \\
\hline & I956 & 19.5 & 17.1 & 17.4 & 18.0 & 1.3 & 1.76 & 1.82 & 1.74 & 1.77 & 0.04 \\
\hline & I958 & 19.9 & 19.8 & 21.1 & 20.3 & 0.7 & 0.90 & 1.00 & 1.00 & 0.97 & 0.06 \\
\hline & I959 & 13.1 & 10.3 & 11.2 & 11.5 & 1.4 & & & & & \\
\hline & I960 & 21.1 & 19.9 & & 20.5 & 0.9 & 1.39 & 1.44 & 1.32 & 1.39 & 0.06 \\
\hline & I961 & 16.0 & 15.0 & 15.4 & 15.4 & 0.5 & & & & & \\
\hline & I963 & 18.8 & 18.4 & 17.4 & 18.2 & 0.7 & 2.21 & 2.23 & 2.19 & 2.21 & 0.02 \\
\hline & I964 & 13.0 & 15.4 & 13.2 & 13.9 & 1.3 & & & & & \\
\hline & I965 & & & & & & & & & & \\
\hline & I966 & 21.3 & 22.1 & 19.0 & 20.8 & 1.6 & 0.80 & 0.48 & 1.04 & 0.77 & 0.28 \\
\hline & I967 & 15.6 & 20.1 & 17.1 & 17.6 & 2.3 & 1.86 & 1.62 & 1.86 & 1.78 & 0.14 \\
\hline & I972 & 19.5 & 14.5 & 17.0 & 17.0 & 2.5 & 1.30 & 1.27 & 1.43 & 1.33 & 0.08 \\
\hline & I973 & 15.7 & 14.9 & 15.1 & 15.2 & 0.4 & 1.23 & 1.31 & 1.37 & 1.30 & 0.07 \\
\hline & I978 & 2.8 & 2.3 & 3.2 & 2.8 & 0.5 & 1.00 & 1.08 & 1.01 & 1.03 & 0.05 \\
\hline & I980 & 14.4 & 12.4 & 12.9 & 13.2 & 1.0 & 2.04 & 1.99 & 1.98 & 2.00 & 0.03 \\
\hline & I981 & 16.9 & 16.0 & 14.4 & 15.8 & 1.3 & 1.37 & 1.41 & 1.43 & 1.40 & 0.03 \\
\hline & I983 & 17.5 & 16.0 & 16.0 & 16.5 & 0.9 & 1.40 & 1.50 & 1.50 & 1.47 & 0.06 \\
\hline & I985 & 9.4 & & & 9.4 & & 2.00 & & & 2.00 & \\
\hline & I986 & & & & & & 1.43 & 1.41 & 1.37 & 1.40 & 0.03 \\
\hline \multirow{5}{*}{ 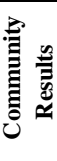 } & & \multicolumn{3}{|c|}{ Consensus Mean } & \multicolumn{2}{|l|}{16.1} & \multicolumn{3}{|c|}{ Consensus Mean } & 1.44 & \\
\hline & & \multicolumn{3}{|c|}{ Consensus Standard Deviation } & 3.0 & & \multicolumn{3}{|c|}{ Consensus Standard Deviation } & 0.38 & \\
\hline & & Maximu & & & 22.7 & & Maximu & & & 2.21 & \\
\hline & & Minimur & & & 2.8 & & Minimur & & & 0.68 & \\
\hline & & $\mathrm{N}$ & & & 39 & & $\mathrm{~N}$ & & & 36 & \\
\hline
\end{tabular}




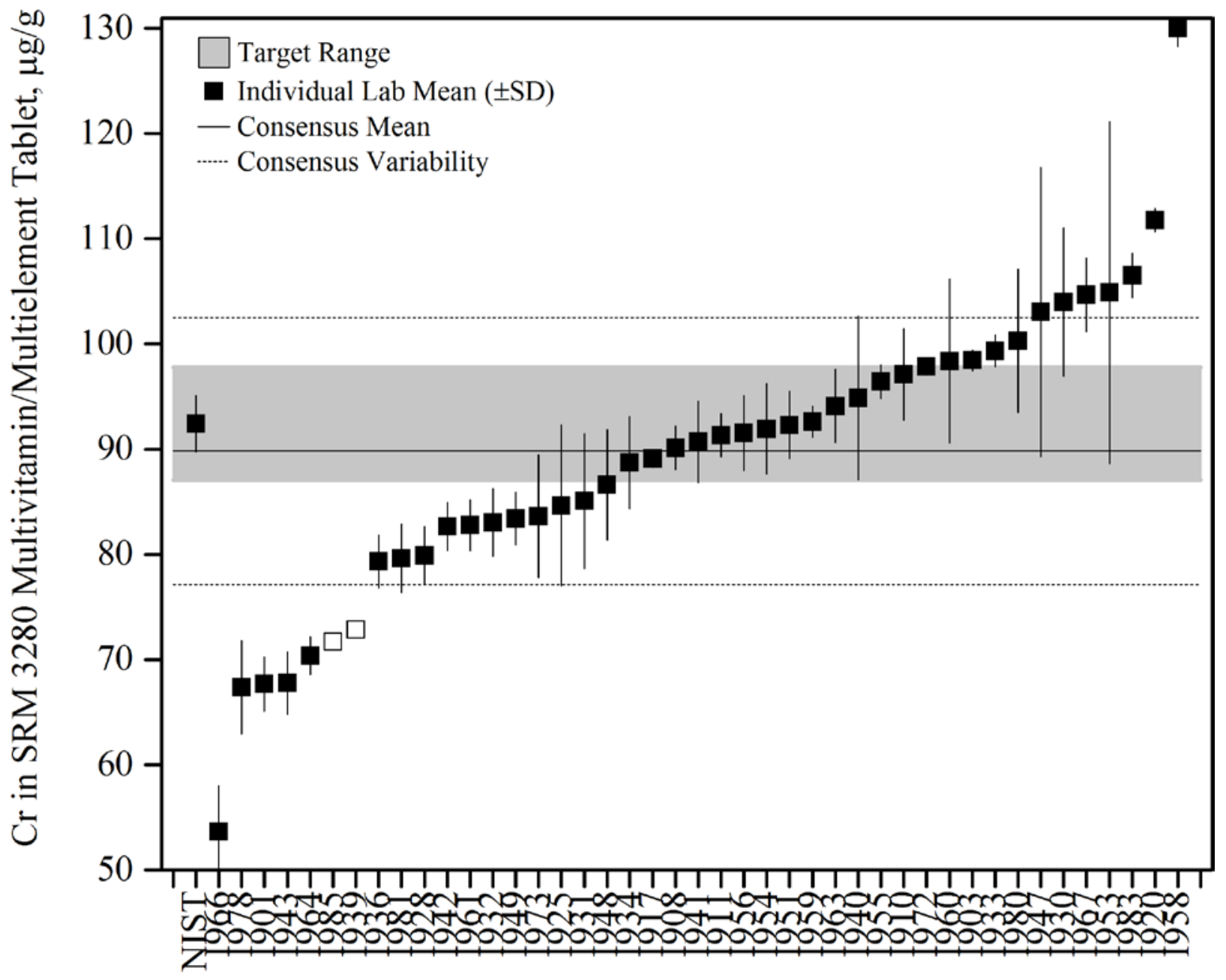

Figure 1. Chromium in SRM 3280 Multivitamin/Multielement Tablets (method comparison data summary view - digestion method). In this view, individual laboratory data are plotted with the individual laboratory standard deviation (error bars). Data points that are unfilled represent laboratories that only reported a single value for that analyte and therefore were not included in the consensus mean. The black solid line represents the consensus mean, and the black dotted lines represent the consensus variability calculated as one standard deviation about the consensus mean. The gray shaded region represents the target zone for "acceptable" performance, which encompasses the NIST certified value bounded by twice its uncertainty $\left(U_{95}\right)$. 


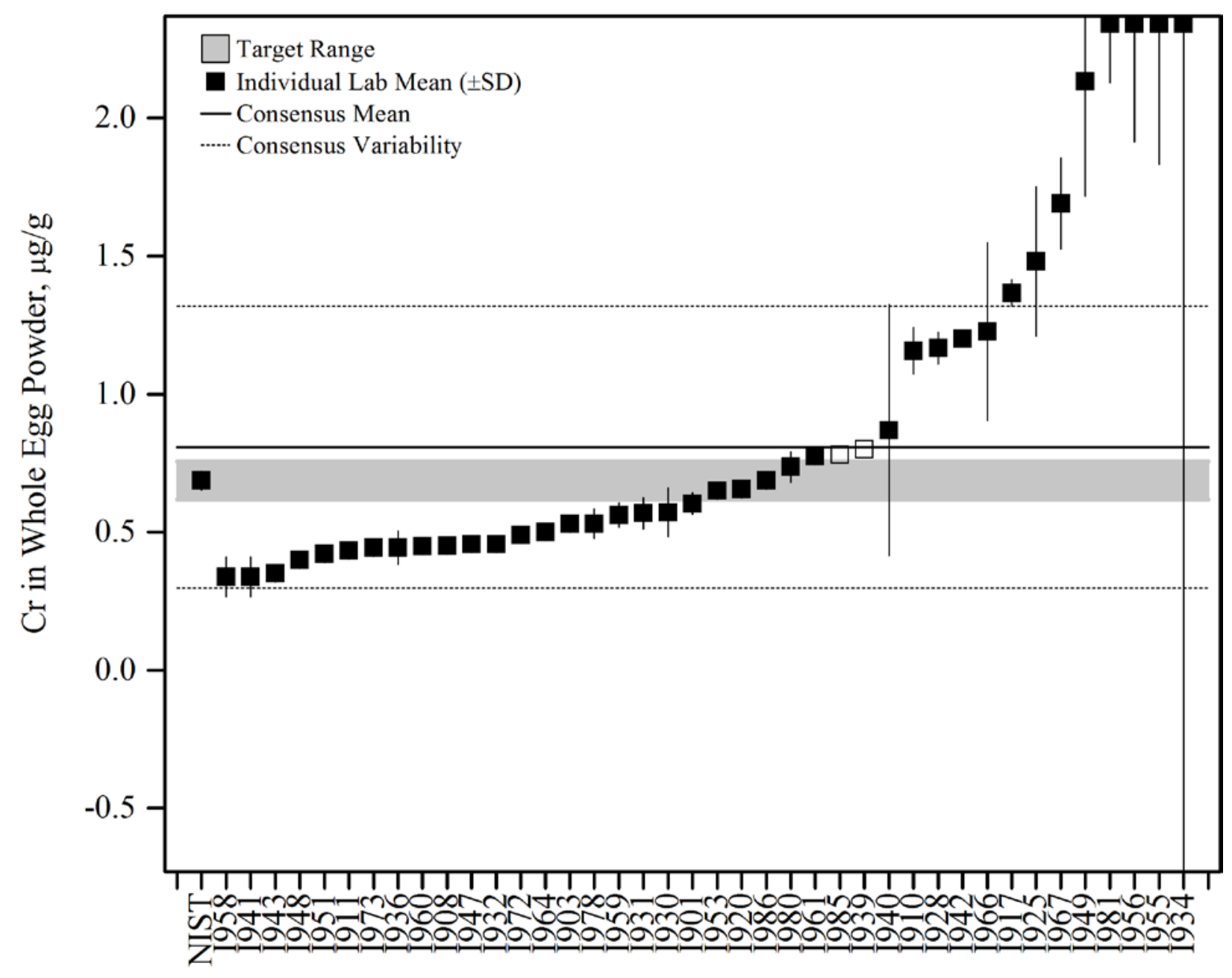

Figure 2. Chromium in whole egg powder (data summary view). In this view, individual laboratory data are plotted with the individual laboratory standard deviation (error bars). Data points that are unfilled represent laboratories that only reported a single value for that analyte and therefore were not included in the consensus mean. The black solid line represents the consensus mean, and the black dotted lines represent the consensus variability calculated as one standard deviation about the consensus mean. The gray shaded region represents the target zone for “acceptable” performance, which encompasses the NIST-assessed value bounded by an uncertainty of $5 \%$. The NIST value is the mean of three results determined by ICP-MS. 


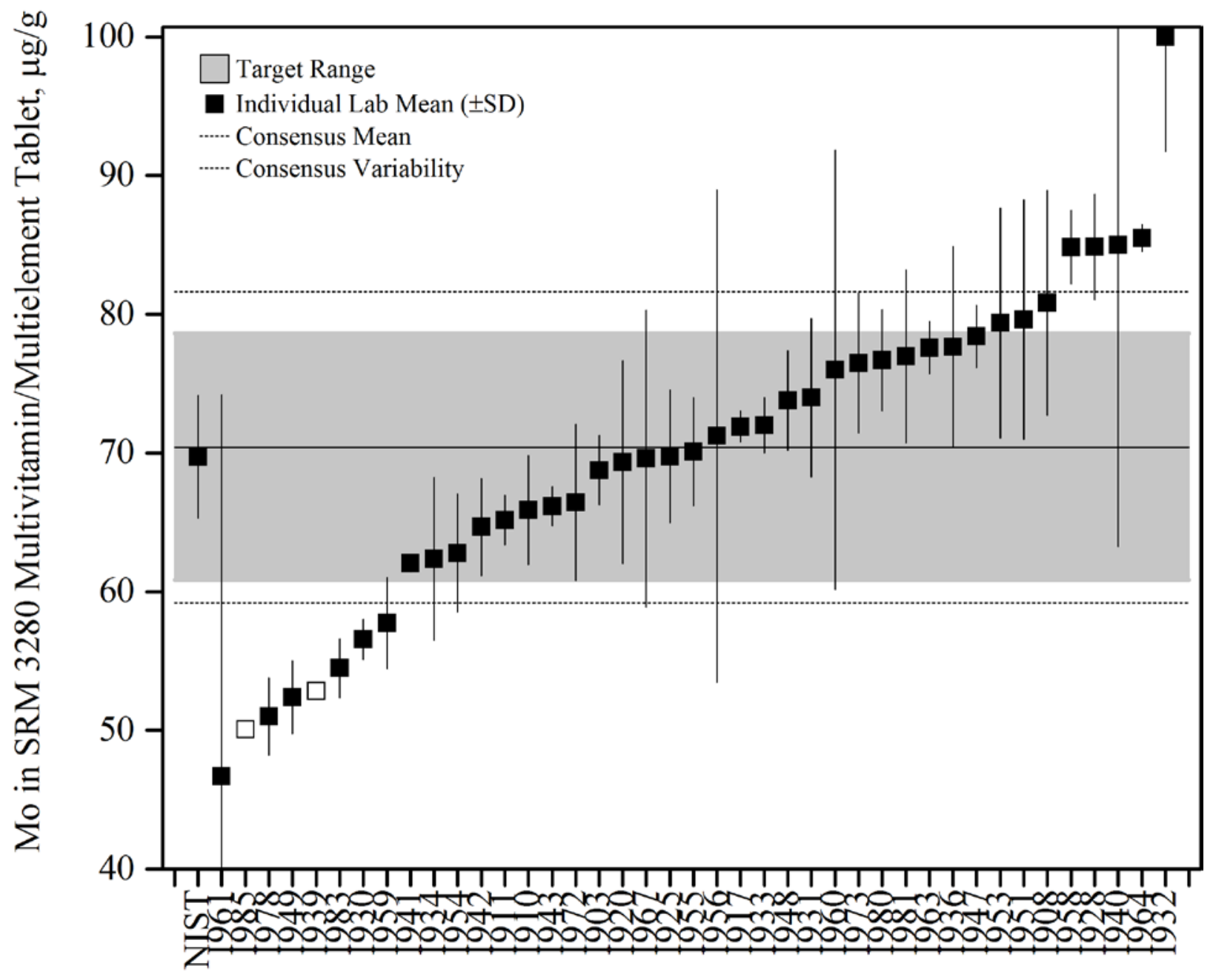

Figure 3. Molybdenum in SRM 3280 Multivitamin/Multielement Tablets (data summary view). In this view, individual laboratory data are plotted with the individual laboratory standard deviation (error bars). Data points that are unfilled represent laboratories that only reported a single value for that analyte and therefore were not included in the consensus mean. The black solid line represents the consensus mean, and the black dotted lines represent the consensus variability calculated as one standard deviation about the consensus mean. The gray shaded region represents the target zone for "acceptable” performance, which encompasses the NIST certified value bounded by twice its uncertainty $\left(U_{95}\right)$. 


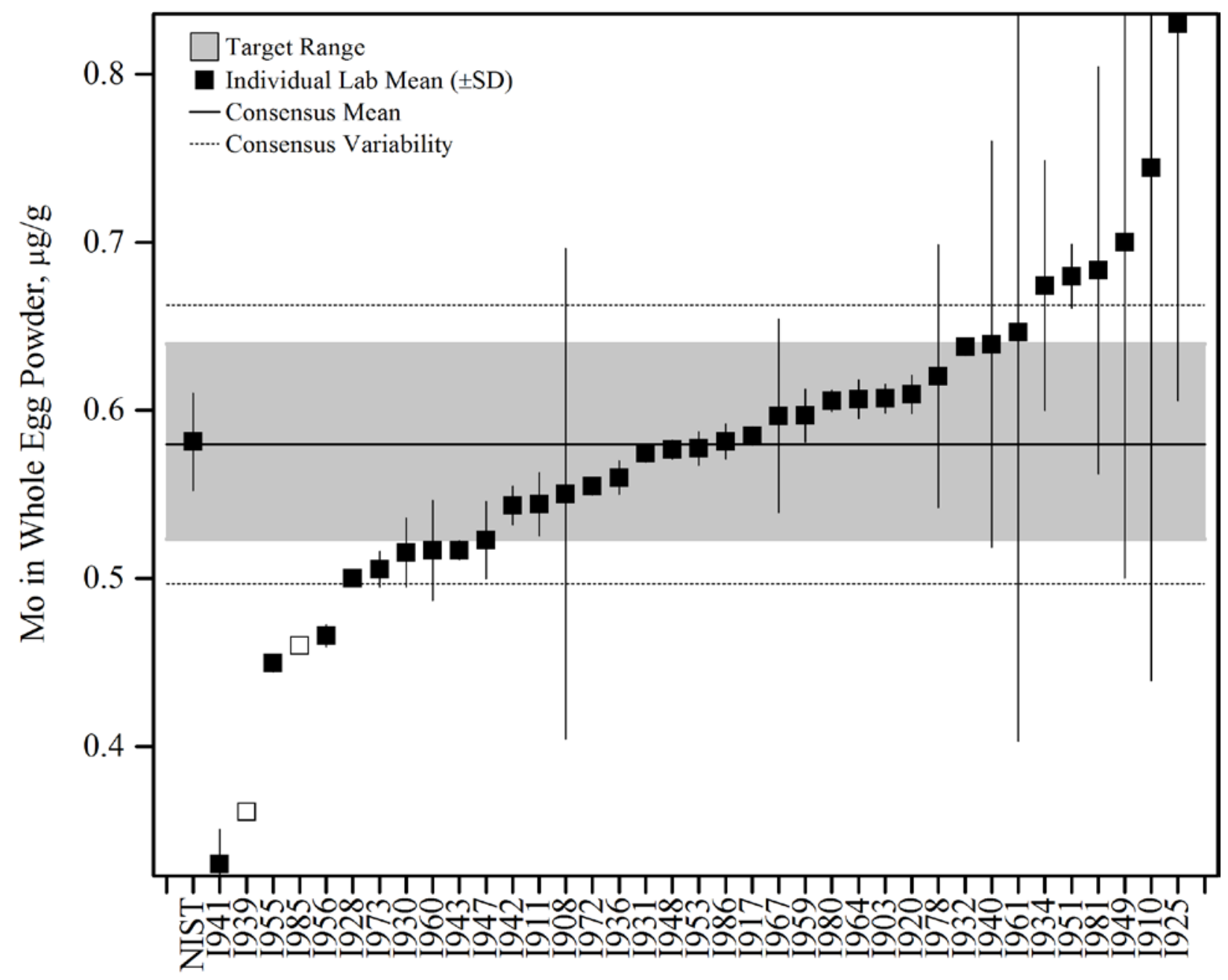

Figure 4. Molybdenum in whole egg powder (data summary view). In this view, individual laboratory data are plotted with the individual laboratory standard deviation (error bars). Data points that are unfilled represent laboratories that only reported a single value for that analyte and therefore were not included in the consensus mean. The black solid line represents the consensus mean, and the black dotted lines represent the consensus variability calculated as one standard deviation about the consensus mean. The gray shaded region represents the target zone for "acceptable" performance, which encompasses the NIST-assessed value bounded by an uncertainty of $5 \%$. The NIST value is the mean three of results determined by ICP-MS. 


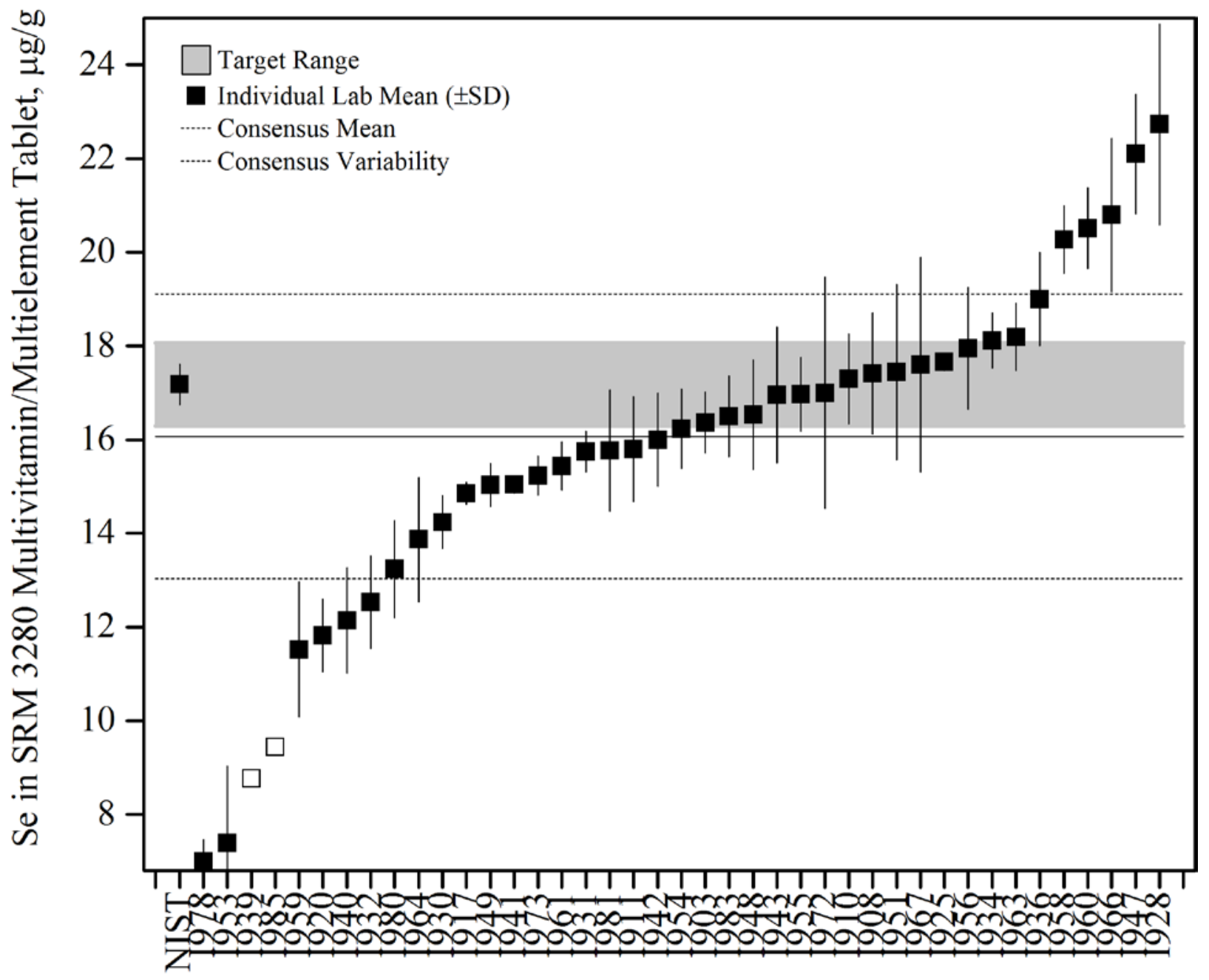

Figure 5. Selenium in SRM 3280 Multivitamin/Multielement Tablets (data summary view). In this view, individual laboratory data are plotted with the individual laboratory standard deviation (error bars). Data points that are unfilled represent laboratories that only reported a single value for that analyte and therefore were not included in the consensus mean. The black solid line represents the consensus mean, and the black dotted lines represent the consensus variability calculated as one standard deviation about the consensus mean. The gray shaded region represents the target zone for "acceptable" performance, which encompasses the NIST certified value bounded by twice its uncertainty $\left(U_{95}\right)$. 


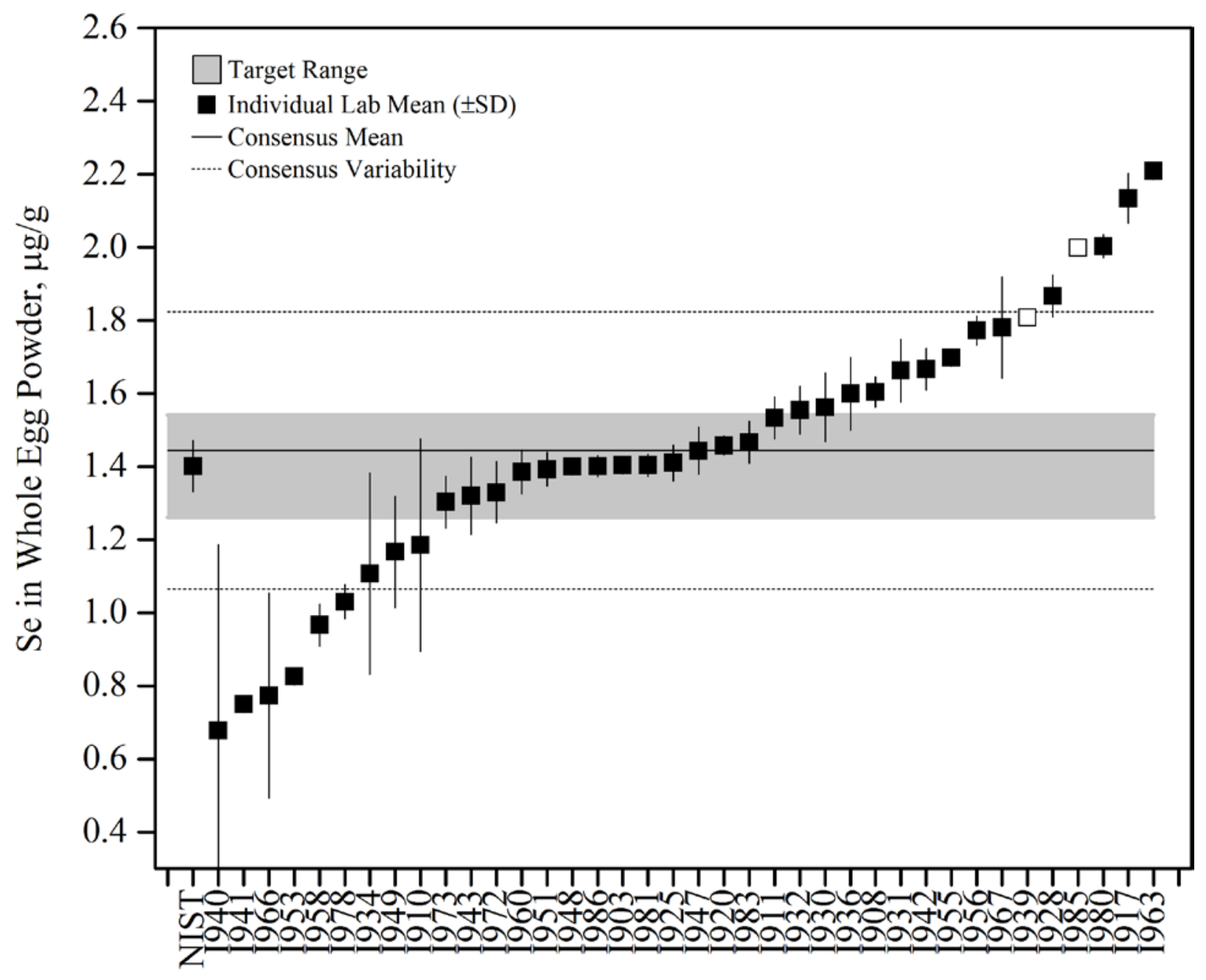

Figure 6. Selenium in whole egg powder (data summary view). In this view, individual laboratory data are plotted with the individual laboratory standard deviation (error bars). Data points that are unfilled represent laboratories that only reported a single value for that analyte and therefore were not included in the consensus mean. The black solid line represents the consensus mean, and the black dotted lines represent the consensus variability calculated as one standard deviation about the consensus mean. The gray shaded region represents the target zone for “acceptable” performance, which encompasses the NIST-assessed value bounded by an uncertainty of $5 \%$. The NIST value is the mean of three results determined by ICP-MS. 


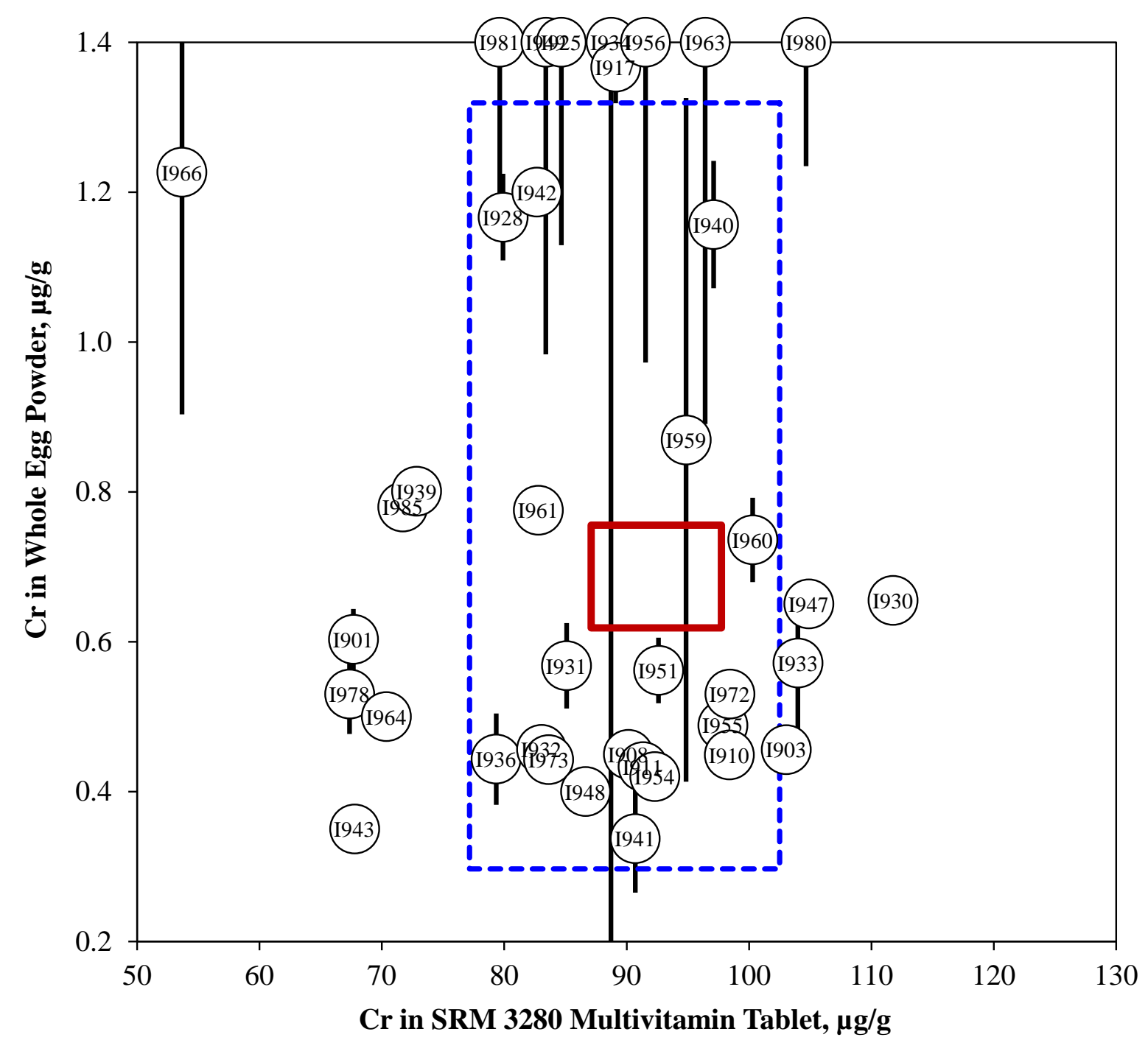

Figure 7. Chromium in whole egg powder and SRM 3280 Multivitamin/Multielement Tablets (sample/control comparison view). In this view, the individual laboratory results for the control (SRM 3280 Multivitamin/Multielement Tablets) with a certified value for the analyte are compared to the results for a sample (whole egg powder). The error bars represent the individual laboratory standard deviation. The solid red lines represent the target zone for the control ( $\mathrm{x}$ axis) and the unknown sample (y-axis). The dotted blue box represents the consensus zone for the control (x-axis) and the unknown sample (y-axis). 


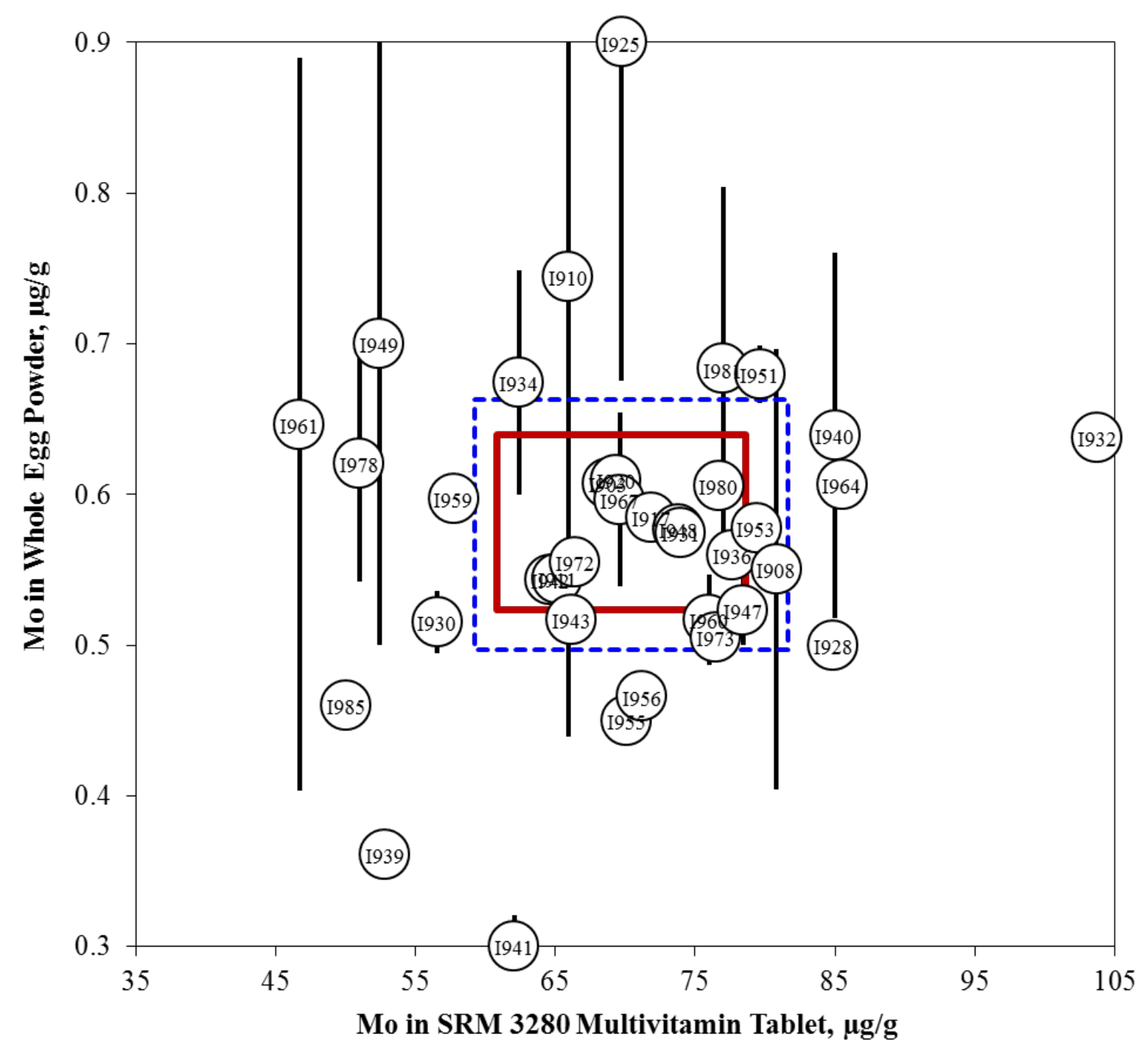

Figure 8. Molybdenum in whole egg powder and SRM 3280 Multivitamin/Multielement Tablets (sample/control comparison view). In this view, the individual laboratory results for the control (SRM 3280 Multivitamin/Multielement Tablets) with a certified value for the analyte are compared to the results for a sample (whole egg powder). The error bars represent the individual laboratory standard deviation. The solid red lines represent the target zone for the control (x-axis) and the unknown sample (y-axis). The dotted blue box represents the consensus zone for the control (x-axis) and the unknown sample (y-axis). 


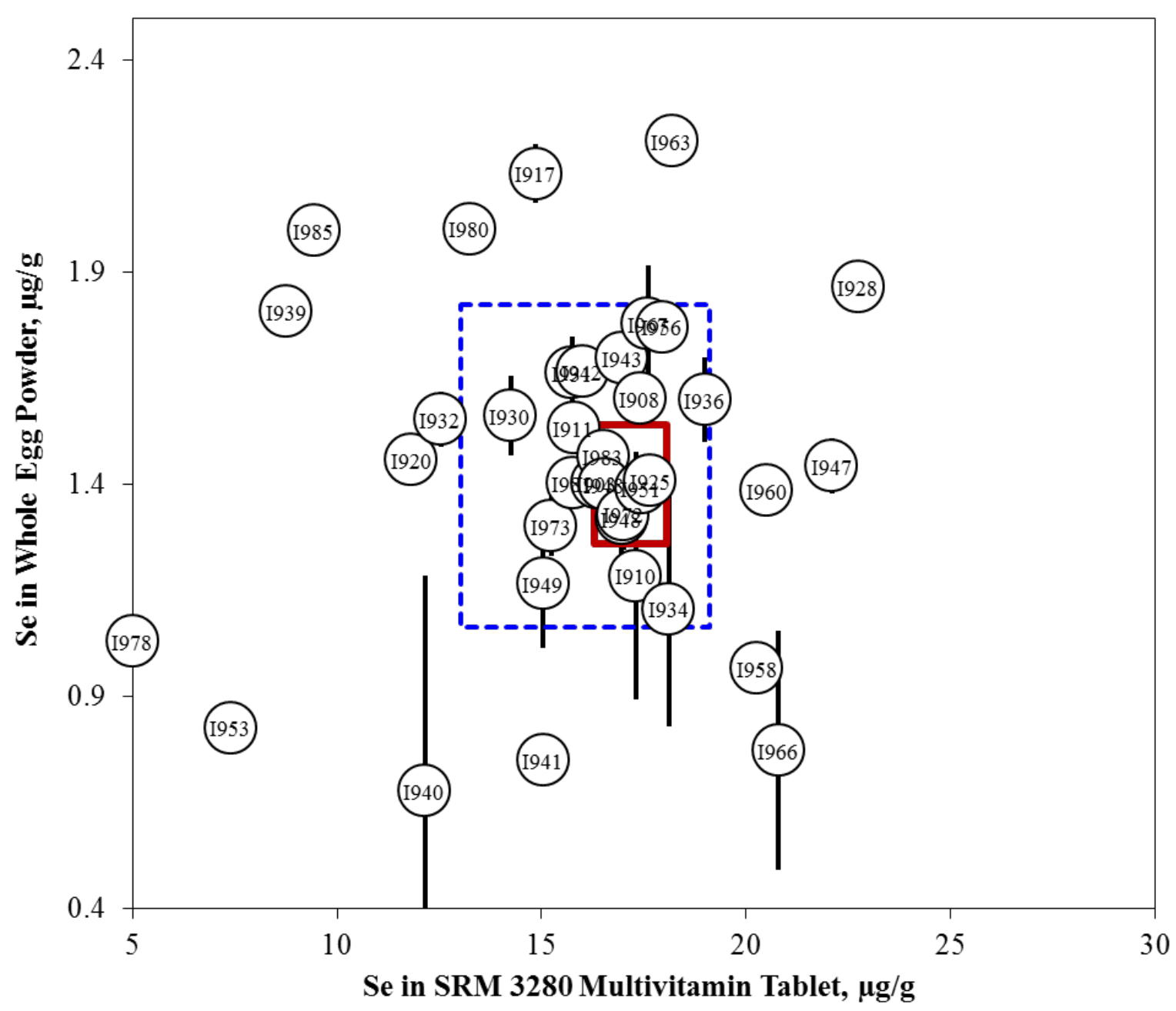

Figure 9. Selenium in whole egg powder and SRM 3280 Multivitamin/Multielement Tablets (sample/control comparison view). In this view, the individual laboratory results for the control (SRM 3280 Multivitamin/Multielement Tablets) with a certified value for the analyte are compared to the results for a sample (whole egg powder). The error bars represent the individual laboratory standard deviation. The solid red lines represent the target zone for the control (x-axis) and the unknown sample (y-axis). The dotted blue box represents the consensus zone for the control (x-axis) and the unknown sample (y-axis). 


\section{TOXIC ELEMENTS (Cd) IN FOODS AND SUPPLEMENTS}

\section{Study Overview}

In this study, participants were provided with two NIST SRMs, SRM 3233 Fortified Breakfast Cereal and candidate SRM 3532 Calcium Dietary Supplement. Participants were asked to use in-house analytical methods to determine the mass fraction of cadmium (Cd) in each of the matrices and report values on an as-received basis.

$\underline{\text { Sample Information }}$

Fortified breakfast cereal. Participants were provided with one packet containing approximately $10 \mathrm{~g}$ of fortified breakfast cereal. This material is a wheat-based fortified flake cereal that was ground to $180 \mu \mathrm{m}$, blended, and packaged. Before use, participants were instructed to mix the contents of the packet thoroughly and use a sample size of at least $0.5 \mathrm{~g}$. Participants were asked to store the material at controlled room temperature, $10^{\circ} \mathrm{C}$ to $30^{\circ} \mathrm{C}$, prepare three samples, and report three values from the single packet provided. Approximate analyte levels were not provided to participants prior to the study. The NIST certified value in SRM 3233 was determined using isotope dilution inductively coupled plasma mass spectrometry (ID-ICP-MS). The certified value for Cd in SRM 3233 is $(81.9 \pm 2.0) \mathrm{ng} / \mathrm{g}$ on a dry-mass basis. Following adjustment for moisture content of the material of $1.70 \%$, the as-received target value for Cd in $\mathrm{SRM} 3233$ is $(80.5 \pm 2.0) \mathrm{ng} / \mathrm{g}$.

Calcium dietary supplement. Participants were provided with one packet containing approximately $10 \mathrm{~g}$ of a powdered calcium dietary supplement. The calcium dietary supplement was prepared from commercially purchased calcium tablets that were ground to $180 \mu \mathrm{m}$, blended, and packaged. Before use, participants were instructed to thoroughly mix the contents of the packet and use a sample size of at least $0.5 \mathrm{~g}$. Participants were asked to store the material at controlled room temperature, $10{ }^{\circ} \mathrm{C}$ to $30{ }^{\circ} \mathrm{C}$, prepare three samples, and report three values from the single packet provided. Approximate analyte levels were not provided to participants prior to the study. The NIST-estimated value for Cd in candidate SRM 3532 was determined by ID-ICP-MS. The estimated value, based on the mean and expanded uncertainty of duplicate measurements from six packets, is $(94.7 \pm 1.7) \mathrm{ng} / \mathrm{g}$.

\section{$\underline{\text { Study Results }}$}

- Fifty-three laboratories enrolled in this exercise and received samples, and forty-two laboratories reported results for Cd (79 \% participation).

- The consensus mean for $\mathrm{Cd}$ in the fortified breakfast cereal was within the target range with an acceptable variability (11\% RSD).

- The consensus mean for Cd in the calcium dietary supplement was slightly below the target range but had acceptable variability (16 \% RSD).

- A majority of the laboratories reported using either microwave digestion (62\%) or open beaker digestion (29\%) for sample preparation. Four laboratories reported using hot block digestion (9\%).

- A majority of the laboratories (88 \%) reported using ICP-MS as their analytical method for analysis. Only four laboratories reported using either ICP-OES or AAS for their analytical measurements for fortified breakfast cereal and five laboratories reported to 
have used either ICP-OES or AAS for their analytical measurements for calcium dietary supplement $(<12 \%)$.

Technical Recommendations

The following recommendations are based on results provided by the participants in this study.

- While twice as many laboratories reported using microwave digestion for sample preparation than other methods reported, there did not seem to be a difference in results based on the sample preparation method used.

- Cadmium can be difficult to measure by ICP-OES because of low sensitivity. Using AAS to measure Cd should not pose any significant problems but sometimes an extraction or separation step is included.

- Spectral interferences can make Cd difficult to measure by ICP-MS if there are high concentrations of certain elements, mainly Mo, Sn, or Zr, but the calcium dietary supplement presents the special case of having a high ratio of $\mathrm{Ca}$ to $\mathrm{Cd}^{4}$.

- A scan of the sample beforehand will indicate if there are potential interferences in the sample that will need to be addressed.

- There can be interferences with commonly used masses of $\mathrm{Cd}\left({ }^{111} \mathrm{Cd},{ }^{112} \mathrm{Cd}\right.$, ${ }^{113} \mathrm{Cd}$, and ${ }^{114} \mathrm{Cd}$ ). Examples of molecular interferences include: ${ }^{95,96,97 \text { and }}$

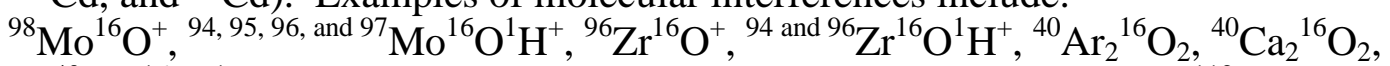
or ${ }^{40} \mathrm{Ca}_{2}{ }^{16} \mathrm{O}_{2}{ }^{1} \mathrm{H}^{+}$; examples of elemental isobaric interferences include: ${ }^{112} \mathrm{Sn}$, ${ }^{113} \mathrm{In}$, and ${ }^{114} \mathrm{Sn}$.

- Chemical separations by anion chromatography can reduce interferences but because of the labor intensive work involved it is usually impractical for laboratories to do a chemical separation on each sample.

- Collision cell technology, available on most newer-model ICP-MS instruments, can be used to remove many of the molecular interferences that may be found in these two materials.

- Interference equations inherent to the software provided on some ICP-MS instruments are designed to correct for interferences, and these equations can also be applied off-line. Both are less labor-intensive alternatives to chemical separations.

- Many ICP-MS instruments run in either pulse mode or analog mode.

- If sample solutions fall outside of the dynamic range for pulse mode, then the instrument will use both the pulse and analog mode. In this case, the ICP-MS must be calibrated for both modes.

- It is often easier and more accurate to ensure that the calibrants are linear in the pulse mode and that the samples are within this linear range.

- As shown in Figure 14, many laboratories reported either high values for both samples or low values for both samples. High values may indicate spectral interference or contamination. Low values may indicate matrix-induced signal suppression which may be avoided with the use of an internal standard. Dilution of sample solutions can also decrease matrix-induced signal suppression as long as solutions are not diluted below the detection limit. Additionally, high or low

\footnotetext{
${ }^{4}$ Murphy, K.E., Vetter, T.W. (2013) Recognizing and overcoming analytical error in the use of ICP-MS for the determination of cadmium in breakfast cereal and dietary supplements. Anal Bioanal Chem 405 4579-4588.
} 
results can be an indication of a calibration error. A calibration curve needs to tightly bracket expected working solutions and be linear in that region. More accurate measurements can be achieved by making sure the sample concentrations fall within the middle of the calibration curve.

- Run a well-documented quality control sample with your unknown samples to ensure your method is performing as expected.

- Double-check all calculations for errors. Compare these to your quality assurance samples to make sure all calculations have been done correctly. 
Table 5. Individual data table (NIST) for cadmium in foods and dietary supplements.

\section{National Institute of Standards \& Technology}

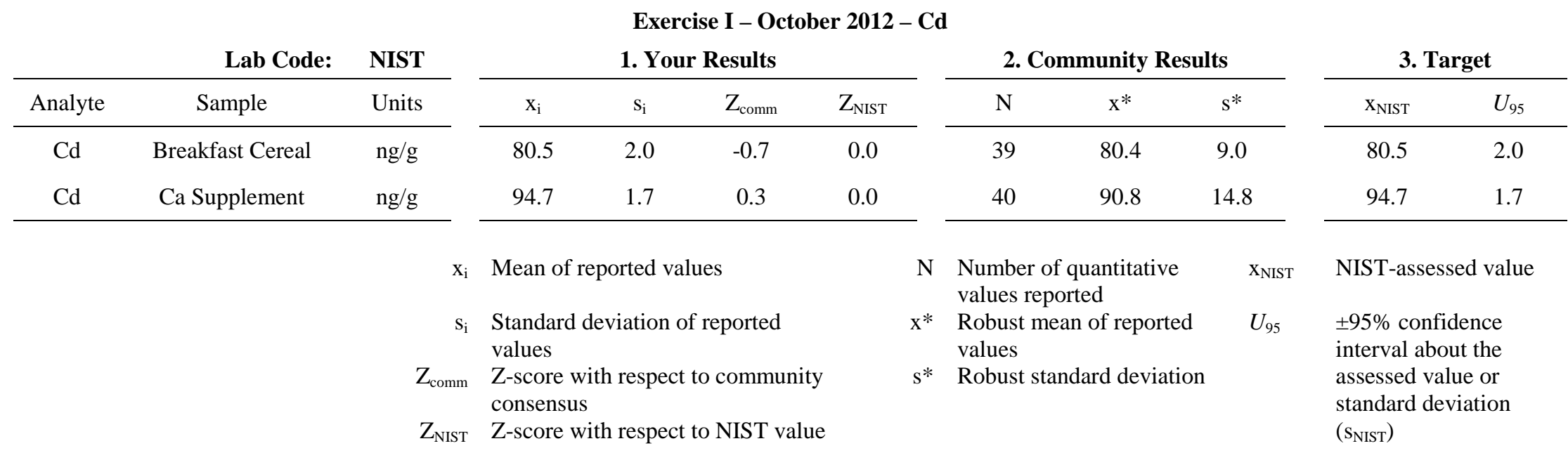


Table 6. Data summary table for cadmium in foods and dietary supplements.

\begin{tabular}{|c|c|c|c|c|c|c|c|c|c|c|c|}
\hline & \multirow[b]{3}{*}{ Lab } & \multicolumn{10}{|c|}{ Cadmium } \\
\hline & & \multicolumn{5}{|c|}{ SRM 3233 Fortified Breakfast Cereal (ng/g) } & \multicolumn{5}{|c|}{ Candidate SRM 3532 Ca Supplement (ng/g) } \\
\hline & & A & B & C & Avg & SD & A & B & C & Avg & SD \\
\hline \multirow{54}{*}{ 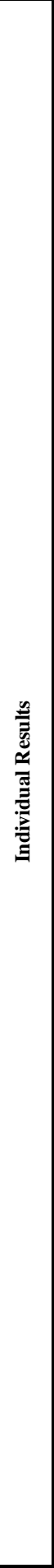 } & NIST & & & & 80.5 & 2.0 & & & & 94.7 & 1.7 \\
\hline & I901 & & & & & & & & & & \\
\hline & I903 & 80.4 & 71.7 & 77.8 & 76.6 & 4.5 & 83.8 & 78.2 & 79.7 & 80.6 & 2.9 \\
\hline & I904 & & & & & & & & & & \\
\hline & I906 & & & & & & & & & & \\
\hline & I907 & & & & & & & & & & \\
\hline & I908 & 72.0 & 70.0 & 78.0 & 73.3 & 4.2 & 79.5 & 81.5 & 84.5 & 81.8 & 2.5 \\
\hline & I910 & 65.0 & 55.5 & 61.9 & 60.8 & 4.8 & 117.0 & 104.0 & 122.0 & 114.3 & 9.3 \\
\hline & I911 & 88.0 & 98.0 & 86.0 & 90.7 & 6.4 & 98.0 & 85.0 & 83.0 & 88.7 & 8.1 \\
\hline & I915 & & & & & & & & & & \\
\hline & I917 & 75.7 & 79.2 & 76.7 & 77.2 & 1.8 & 86.9 & 86.9 & 86.9 & 86.9 & 0.0 \\
\hline & I920 & 108.0 & 96.0 & 100.0 & 101.3 & 6.1 & 107.0 & 114.0 & 110.0 & 110.3 & 3.5 \\
\hline & I925 & 78.4 & 80.8 & 79.9 & 79.7 & 1.2 & 95.1 & 94.5 & 93.1 & 94.2 & 1.0 \\
\hline & I928 & 65.0 & 70.0 & 70.0 & 68.3 & 2.9 & 72.0 & 66.0 & 72.0 & 70.0 & 3.5 \\
\hline & I929 & 144.0 & 145.0 & 146.0 & 145.0 & 1.0 & 128.0 & 129.0 & 130.0 & 129.0 & 1.0 \\
\hline & I930 & 72.2 & 76.3 & 74.4 & 74.3 & 2.1 & 87.9 & 81.0 & 81.1 & 83.3 & 4.0 \\
\hline & I931 & 100.0 & 107.0 & 111.0 & 106.0 & 5.6 & 66.5 & 57.4 & 59.5 & 61.1 & 4.8 \\
\hline & I932 & 81.3 & 77.1 & 82.8 & 80.4 & 3.0 & 99.2 & 96.7 & 98.7 & 98.2 & 1.4 \\
\hline & I933 & 74.0 & 74.0 & 72.0 & 73.3 & 1.2 & 84.0 & 82.0 & 84.0 & 83.3 & 1.2 \\
\hline & I934 & 126.0 & 97.4 & 115.6 & 113.0 & 14.4 & 119.2 & 139.2 & 103.5 & 120.6 & 17.9 \\
\hline & I935 & & & & & & & & & & \\
\hline & I936 & 80.0 & 85.0 & 85.0 & 83.3 & 2.9 & 91.0 & 100.0 & 100.0 & 97.0 & 5.2 \\
\hline & I938 & & & & & & & & & & \\
\hline & I939 & 52.0 & & & 52.0 & & 58.0 & & & 58.0 & \\
\hline & I940 & 76.7 & 84.3 & 73.2 & 78.1 & 5.6 & 63.1 & 69.3 & 99.0 & 77.1 & 19.1 \\
\hline & I942 & 77.0 & 81.0 & 88.0 & 82.0 & 5.6 & 75.0 & 74.0 & 76.0 & 75.0 & 1.0 \\
\hline & I943 & 84.0 & 79.0 & 81.0 & 81.3 & 2.5 & 96.0 & 92.0 & 91.0 & 93.0 & 2.6 \\
\hline & I944 & & & & & & & & & & \\
\hline & I945 & 83.7 & 84.6 & 84.4 & 84.2 & 0.5 & 98.1 & 98.3 & 94.2 & 96.8 & 2.3 \\
\hline & I947 & 89.9 & 86.3 & 83.5 & 86.5 & 3.2 & 96.7 & 99.2 & 92.6 & 96.1 & 3.3 \\
\hline & I948 & 100.0 & 90.0 & 90.0 & 93.3 & 5.8 & 110.0 & 100.0 & 110.0 & 106.7 & 5.8 \\
\hline & I949 & & & & & & & & & & \\
\hline & I950 & 58.7 & 58.1 & 57.6 & 58.1 & 0.6 & 61.5 & 62.7 & 63.6 & 62.6 & 1.1 \\
\hline & I951 & 83.3 & 90.8 & 85.3 & 86.5 & 3.9 & 95.6 & 93.9 & 100.1 & 96.5 & 3.2 \\
\hline & I953 & 72.0 & 78.0 & 74.0 & 74.7 & 3.1 & 91.0 & 91.0 & 92.0 & 91.3 & 0.6 \\
\hline & I954 & 80.0 & 84.0 & 80.0 & 81.3 & 2.3 & 90.0 & 97.0 & 91.0 & 92.7 & 3.8 \\
\hline & I955 & 73.5 & 72.4 & 75.4 & 73.8 & 1.5 & 78.2 & 81.0 & 80.5 & 79.9 & 1.5 \\
\hline & I956 & 75.2 & 76.3 & 76.4 & 75.9 & 0.7 & 78.3 & 82.0 & 81.1 & 80.5 & 2.0 \\
\hline & I958 & 74.0 & 80.0 & 78.0 & 77.3 & 3.1 & 110.0 & 99.0 & 124.0 & 111.0 & 12.5 \\
\hline & I959 & 80.6 & 77.7 & 76.7 & 78.3 & 2.0 & 100.7 & 95.1 & 94.1 & 96.6 & 3.6 \\
\hline & I960 & 83.3 & 77.5 & 85.4 & 82.1 & 4.1 & 86.0 & 102.3 & 90.7 & 93.0 & 8.4 \\
\hline & I961 & 162.2 & 127.2 & 112.4 & 134.0 & 25.6 & 254.9 & 204.7 & 211.6 & 223.7 & 27.2 \\
\hline & I962 & & & & & & 74.6 & 69.7 & 77.6 & 74.0 & 4.0 \\
\hline & I963 & 85.4 & 85.6 & 81.4 & 84.1 & 2.4 & 101.3 & 93.7 & 96.4 & 97.2 & 3.8 \\
\hline & I964 & 72.0 & 75.0 & 71.0 & 72.7 & 2.1 & 81.0 & 81.0 & 84.0 & 82.0 & 1.7 \\
\hline & I966 & & & & & & & & & & \\
\hline & I967 & 81.4 & 78.0 & 78.2 & 79.2 & 1.9 & 98.0 & 97.8 & 97.1 & 97.6 & 0.5 \\
\hline & I972 & 49.3 & 48.5 & 48.6 & 48.8 & 0.4 & 60.2 & 61.1 & 73.0 & 64.8 & 7.2 \\
\hline & I973 & 77.7 & 68.8 & 71.5 & 72.7 & 4.6 & 87.8 & 80.0 & 89.3 & 85.7 & 5.0 \\
\hline & I978 & 86.0 & 85.0 & 87.1 & 86.0 & 1.1 & 94.5 & 90.0 & 81.4 & 88.6 & 6.7 \\
\hline & I979 & & & & & & & & & & \\
\hline & I980 & 80.0 & 79.0 & 77.0 & 78.7 & 1.5 & 98.0 & 90.0 & 92.0 & 93.3 & 4.2 \\
\hline & I983 & 73.0 & 79.0 & 89.0 & 80.3 & 8.1 & 95.0 & 92.0 & 98.0 & 95.0 & 3.0 \\
\hline & I985 & 57.0 & & & 57.0 & & 62.0 & & & 62.0 & \\
\hline 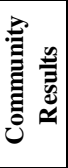 & & $\begin{array}{l}\text { Consenst } \\
\text { Consens } \\
\text { Maximun } \\
\text { Minimum } \\
\mathrm{N}\end{array}$ & $\begin{array}{l}\text { lean } \\
\text { tandard }\end{array}$ & ation & $\begin{array}{c}80.4 \\
9.0 \\
145.0 \\
48.8 \\
39\end{array}$ & & $\begin{array}{l}\text { Consens } \\
\text { Consens } \\
\text { Maximur } \\
\text { Minimum } \\
\text { N }\end{array}$ & $\begin{array}{l}\text { ean } \\
\text { andard }\end{array}$ & lation & $\begin{array}{c}90.8 \\
14.8 \\
223.7 \\
58.0 \\
40\end{array}$ & \\
\hline
\end{tabular}




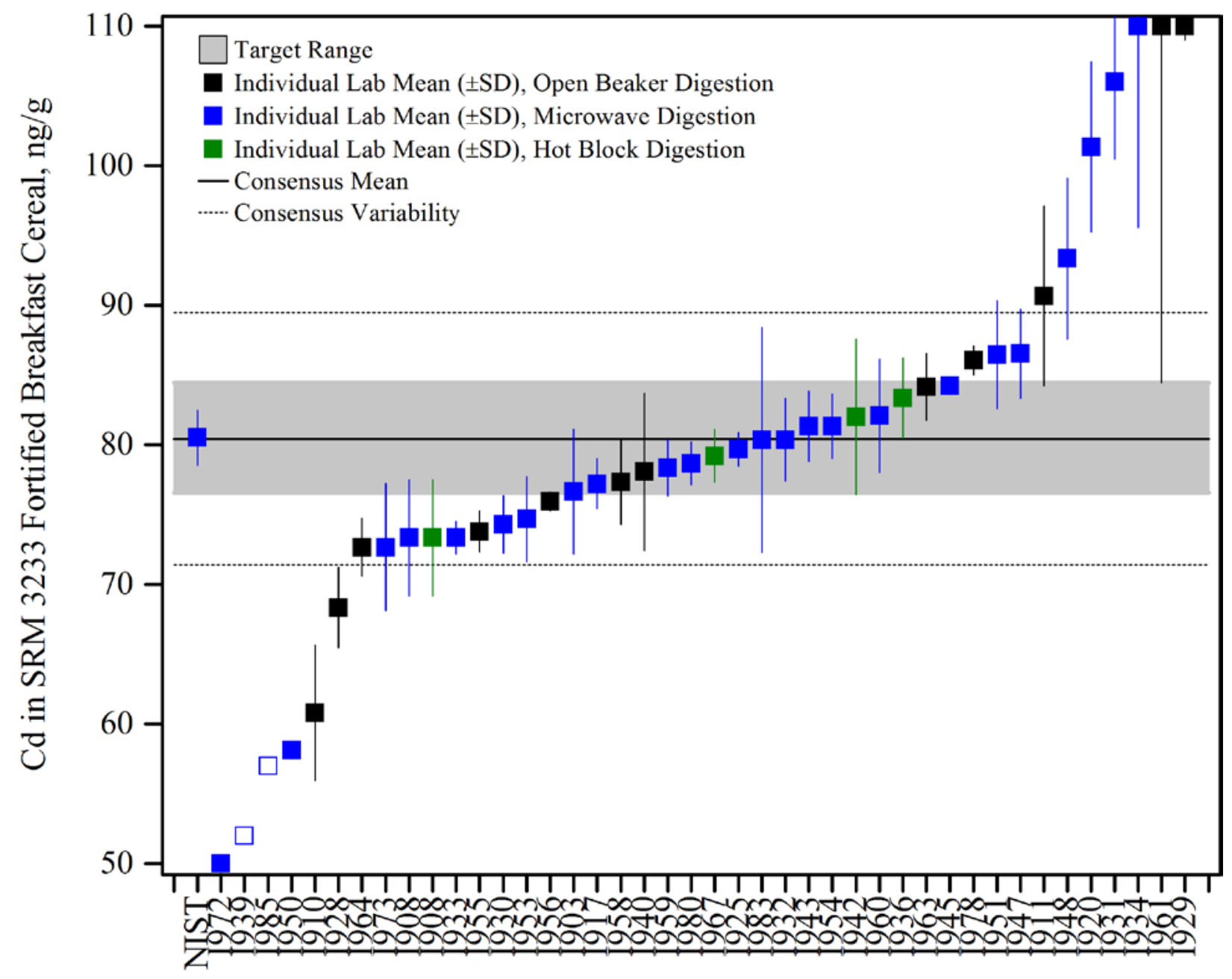

Figure 10. Cadmium in SRM 3233 Fortified Breakfast Cereal (method comparison data summary view - digestion method). In this view, individual laboratory data are plotted with the individual laboratory standard deviation (error bars). The color of the data point represents the sample preparation (digestion) procedure employed. Data points that are unfilled represent laboratories that only reported a single value for that analyte and therefore were not included in the consensus mean. The black solid line represents the consensus mean, and the black dotted lines represent the consensus variability calculated as one standard deviation about the consensus mean. The gray shaded region represents the target zone for "acceptable” performance, which encompasses the NIST certified value bounded by twice its uncertainty $\left(U_{95}\right)$. 


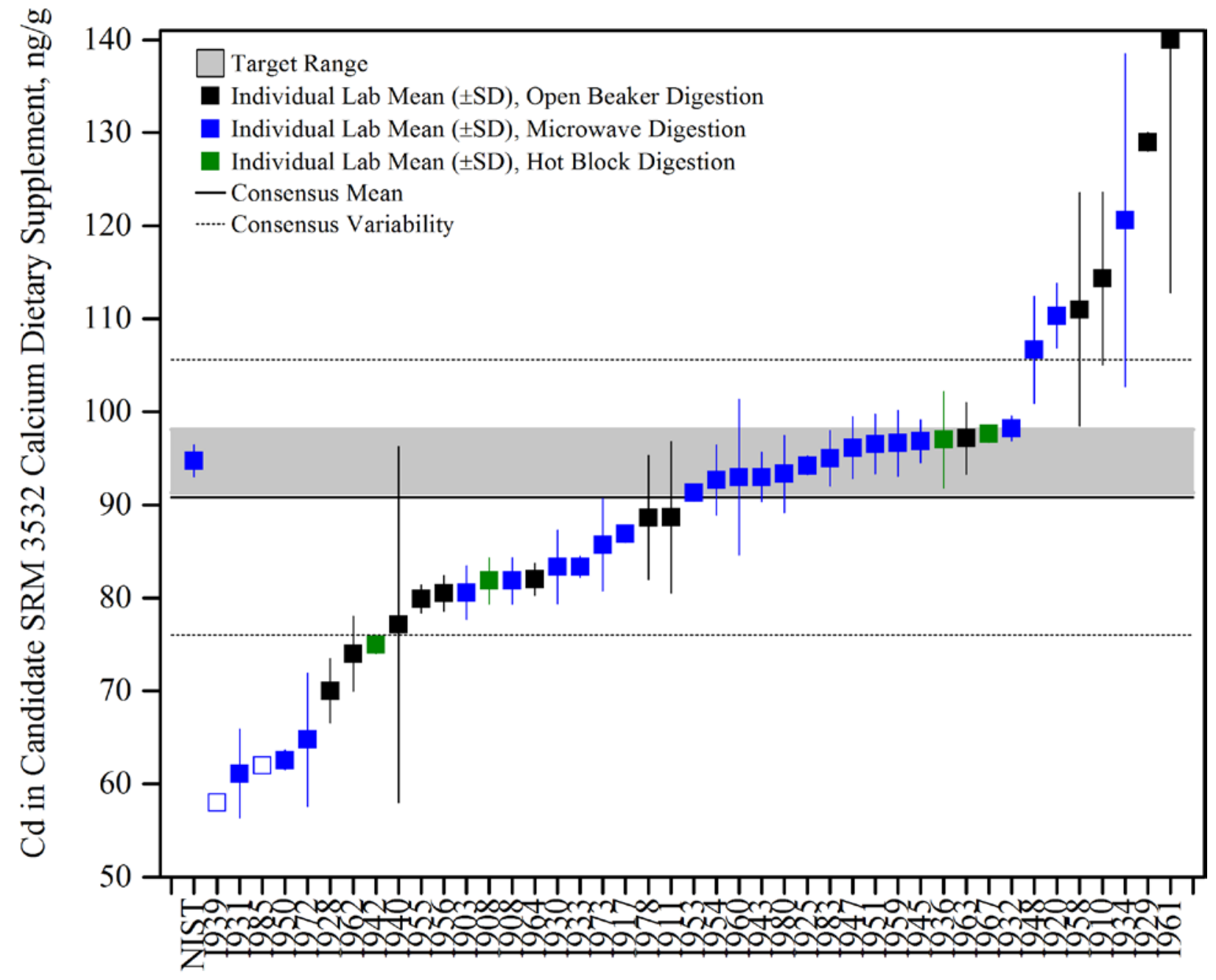

Figure 11. Cadmium in candidate SRM 3532 Calcium Dietary Supplement (method comparison data summary view - digestion method). In this view, individual laboratory data are plotted with the individual laboratory standard deviation (error bars). The color of the data point represents the sample preparation (digestion) procedure employed. Data points that are unfilled represent laboratories that only reported a single value for that analyte and therefore were not included in the consensus mean. The black solid line represents the consensus mean, and the black dotted lines represent the consensus variability calculated as one standard deviation about the consensus mean. The gray shaded region represents the target zone for "acceptable” performance, which encompasses the NIST value determined by ID-ICP-MS, bounded by twice the estimated uncertainty observed for six duplicate measurements. 


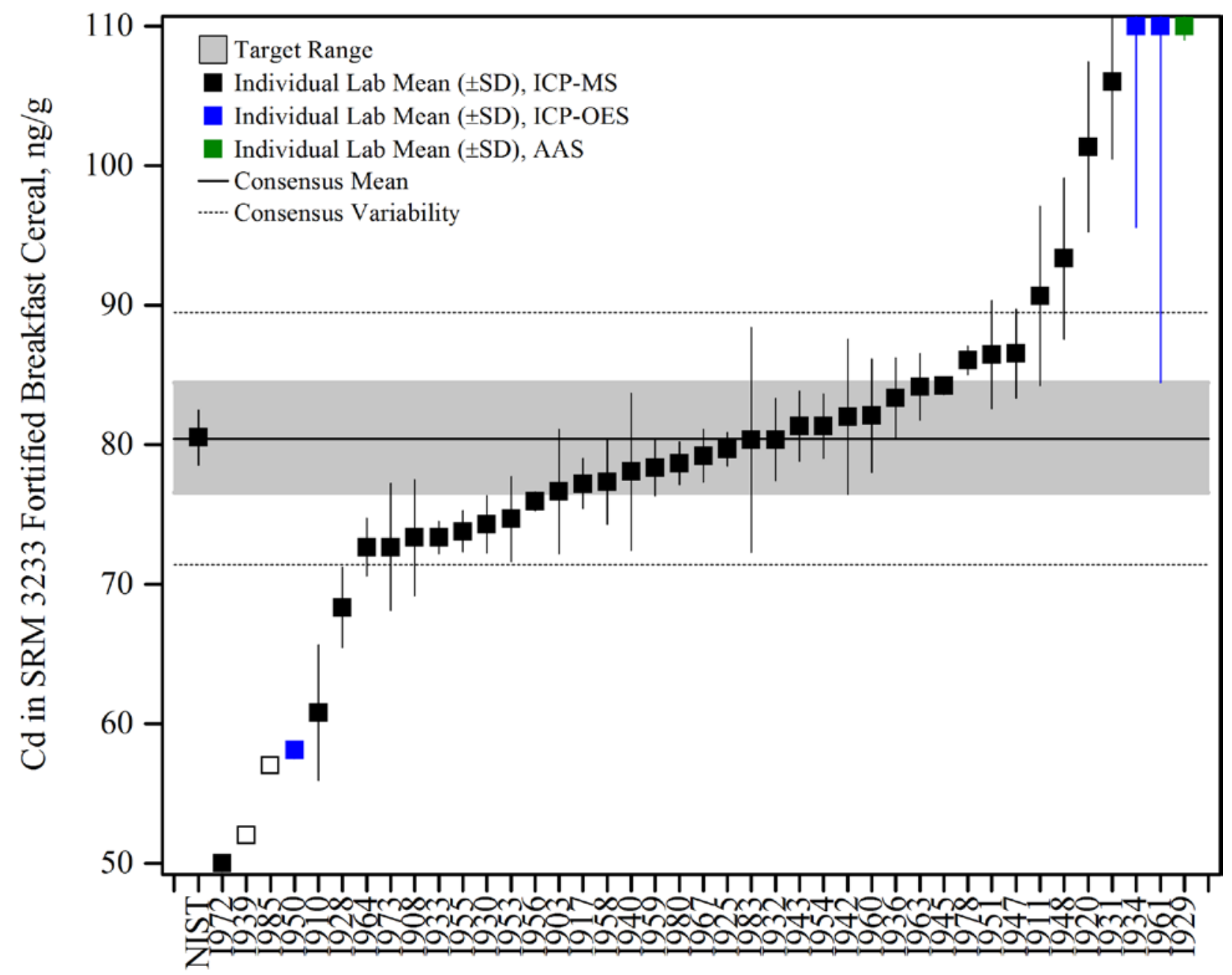

Figure 12. Cadmium in 3233 Fortified Breakfast Cereal (method comparison data summary view - instrumental method). In this view, individual laboratory data are plotted with the individual laboratory standard deviation (error bars). The color of the data point represents the instrumental method employed. Data points that are unfilled represent laboratories that only reported a single value for that analyte and therefore were not included in the consensus mean. The black solid line represents the consensus mean, and the black dotted lines represent the consensus variability calculated as one standard deviation about the consensus mean. The gray shaded region represents the target zone for "acceptable" performance, which encompasses the NIST certified value bounded by twice its uncertainty $\left(U_{95}\right)$. 


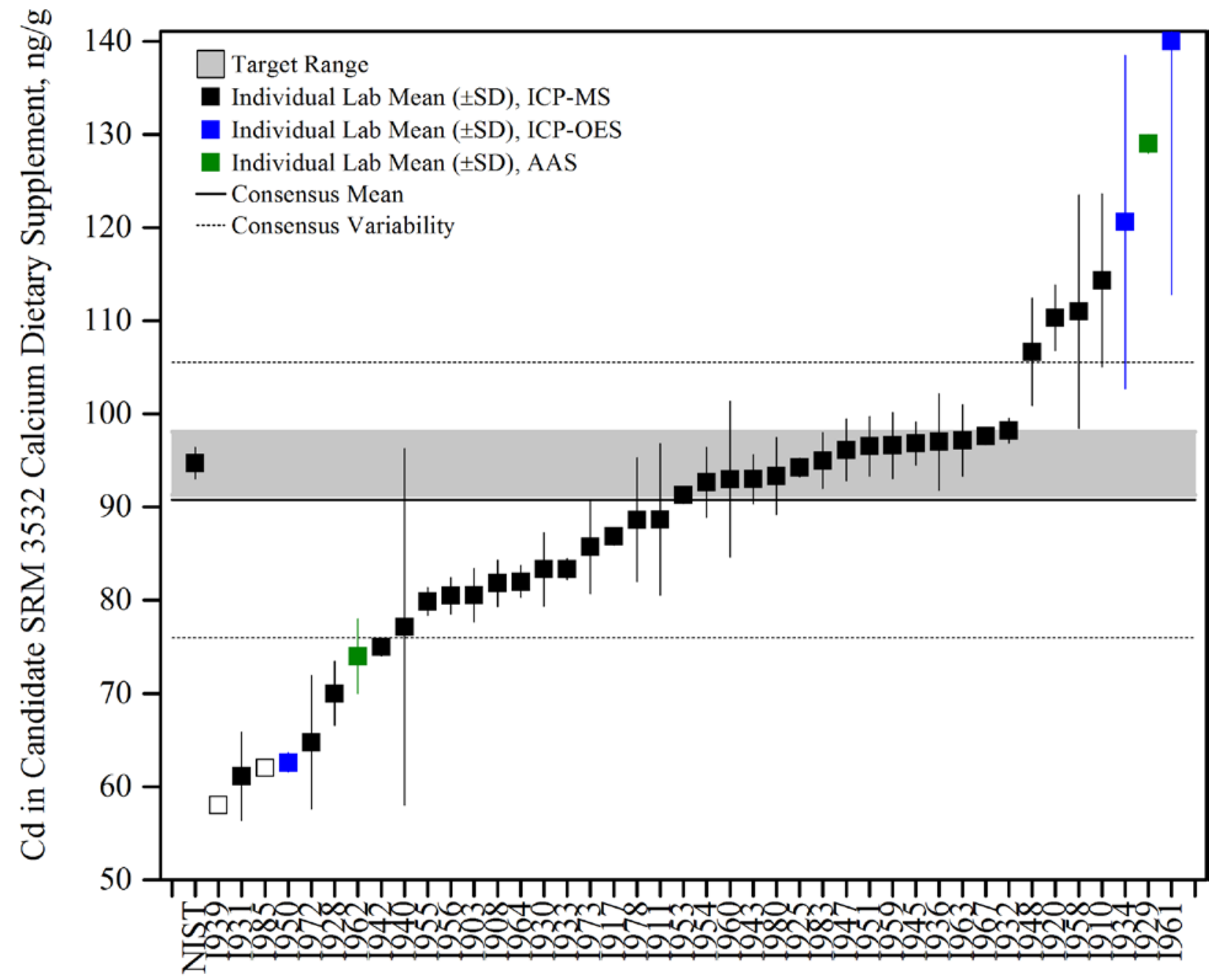

Figure 13. Cadmium in candidate SRM 3532 Calcium Dietary Supplement (method comparison data summary view - instrumental method). In this view, individual laboratory data are plotted with the individual laboratory standard deviation (error bars). The color of the data point represents the instrumental method employed. Data points that are unfilled represent laboratories that only reported a single value for that analyte and therefore were not included in the consensus mean. The black solid line represents the consensus mean, and the black dotted lines represent the consensus variability calculated as one standard deviation about the consensus mean. The gray shaded region represents the target zone for "acceptable" performance, which encompasses the NIST value determined by ID-ICP-MS, bounded by twice the estimated uncertainty observed for six duplicate measurements. 


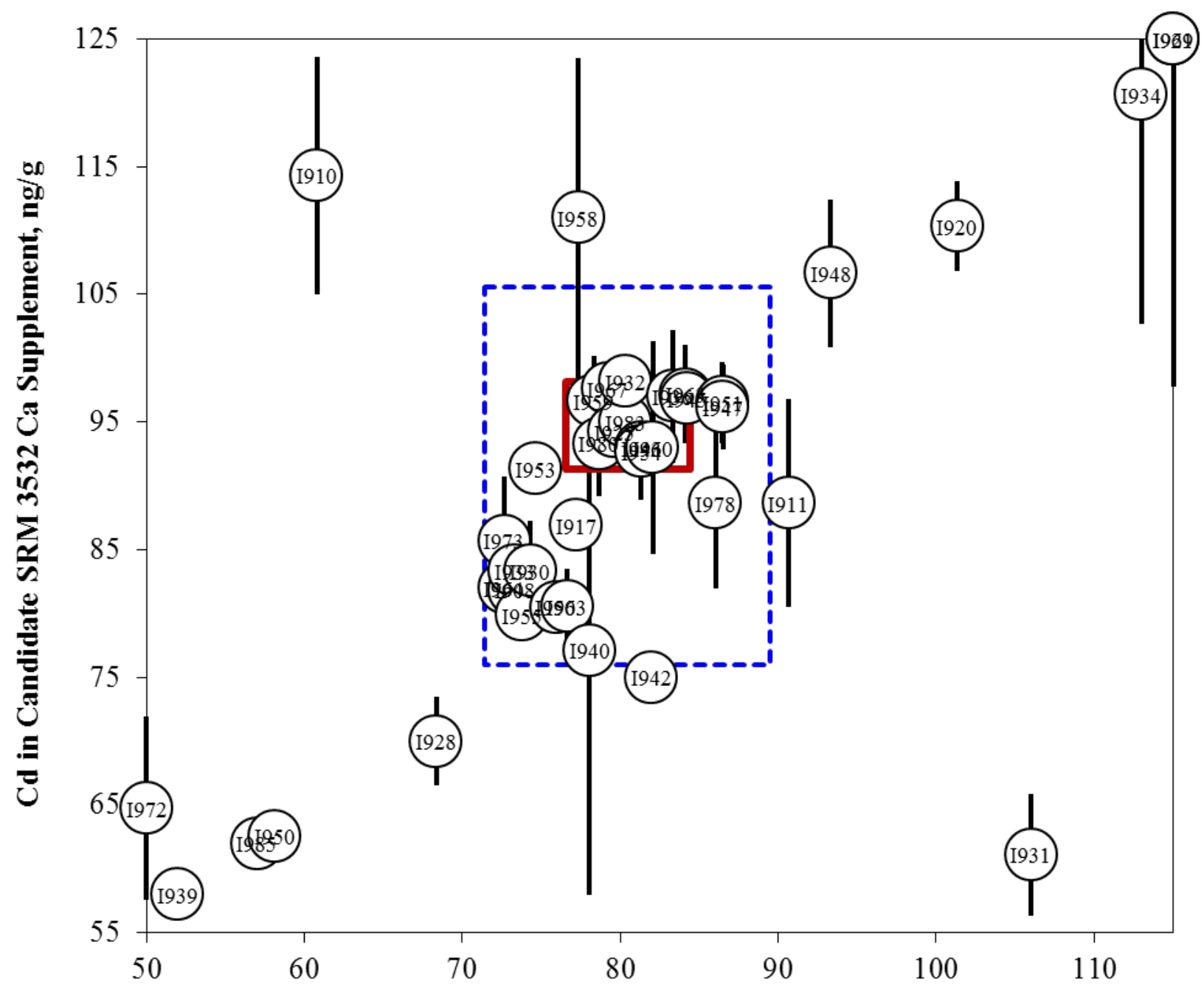

Cd in SRM 3233 Breakfast Cereal, ng/g

Figure 14. Cadmium in candidate SRM 3532 Calcium Dietary Supplement and SRM 3233 Fortified Breakfast Cereal (sample/control comparison view). In this view, the individual laboratory results for the control (SRM 3233 Fortified Breakfast Cereal) with a certified value for the analyte are compared to the results for a sample (candidate SRM 3532 Calcium Dietary Supplement). The error bars represent the individual laboratory standard deviation. The solid red lines represent the target zone for the control (x-axis) and the unknown sample (y-axis). The dotted blue box represents the consensus zone for the control (x-axis) and the unknown sample (y-axis). 


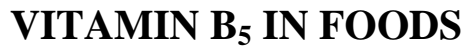

\section{Study Overview}

In this study, participants were provided with two NIST SRMs, SRM 3234 Soy Flour and SRM 3287 Blueberries, neither of which has been fortified with vitamin $B_{5}$ (pantothenic acid). Participants were asked to use in-house analytical methods to determine the mass fractions of vitamin $B_{5}$ in each of the matrices and report values on an as-received basis. Participants were asked to report the vitamin $B_{5}$ content as pantothenic acid; NIST values are reported as pantothenic acid.

\section{Sample Information}

Soy Flour. Participants were provided one packet containing approximately $15 \mathrm{~g}$ of defatted soy flour. The flour was heat-sealed inside $0.1 \mathrm{~mm}$ (4 mil) polyethylene bags, which were then sealed inside Mylar bags. Before use, participants were instructed to thoroughly mix and homogenize the contents of the packet and use a sample size of at least $2 \mathrm{~g}$. Participants were asked to store the soy flour at controlled room temperature, $10^{\circ} \mathrm{C}$ to $30^{\circ} \mathrm{C}$, prepare three samples, and report three values from the single packet provided. Approximate analyte levels were not provided to participants in the study. The NIST certified value for pantothenic acid in SRM 3234 was determined using acidic solvent extraction followed by ID-LC-MS/MS with confirmation using data from external collaborating laboratories. The certified value for pantothenic acid in SRM 3234 is $(11.45 \pm 0.12) \mathrm{mg} / \mathrm{kg}$ on a dry-mass basis. After adjustment for moisture content of the material of $6.13 \%$, the as-received target value for pantothenic acid in SRM 3234 is $(10.75 \pm 0.11) \mathrm{mg} / \mathrm{kg}$.

Blueberries. Participants were provided with three packets, each containing approximately $5 \mathrm{~g}$ of freeze-dried, powdered blueberries. The blueberries were blended, aliquotted, and heat-sealed inside nitrogen-flushed $0.1 \mathrm{~mm}$ (4 mil) polyethylene bags, which were then sealed inside nitrogen-flushed aluminized plastic bags along with two packets of silica gel each. Before use, participants were instructed to thoroughly mix and homogenize the contents of the packet and use a sample size of at least $2.5 \mathrm{~g}$. Participants were also notified that this material was packaged as a powder, but that over time the powder may become a solid mass. For hardened samples, participants were instructed to remove an appropriate test portion using a knife. Participants were asked to report a single value from each packet provided and to store the blueberries at controlled room temperature, $10^{\circ} \mathrm{C}$ to $30^{\circ} \mathrm{C}$. Approximate analyte levels were not provided to participants prior to the study. The NIST certified value for pantothenic acid in SRM 3287 was determined using acidic solvent extraction followed by ID-LC-MS in combination with data from external collaborating laboratories. The certified value of pantothenic acid in SRM 3287 is $(3.36 \pm 0.19) \mathrm{mg} / \mathrm{kg}$. After adjustment for moisture content of the material of $1.41 \%$, the asreceived target value for pantothenic acid in SRM 3287 is $(3.31 \pm 0.19) \mathrm{mg} / \mathrm{kg}$.

\section{Study Results}

- Thirty-three laboratories enrolled in this exercise and received samples, and thirteen laboratories reported results for the soy flour and/or blueberries (39\% participation).

- For both materials, the consensus ranges were very wide. For the soy flour, the consensus mean was higher than the NIST target range, while the consensus mean for the blueberries was contained within the NIST target range (Figure 15 and Figure 16). 
- The dispersion of the data could be a result of challenges in completely extracting the pantothenic acid from the samples or from chromatographic coelutions.

- In the soy flour, nine of the thirteen laboratories (69\%) reported values that were reasonably close to the target range. Three of the remaining four laboratories reported values that were significantly higher than the target range (10 times higher and almost 300 times higher). This could indicate an interference in the analytical method (LC-absorbance with external standard calibration) caused by matrix components. When using a low wavelength (205 nm to $210 \mathrm{~nm}$ ) to detect pantothenic acid (a molecule without a strong chromophore), the method will be highly susceptible to matrix interferences. More information is needed about the analytical methods to draw more conclusions.

- In the blueberries, eight of the eleven laboratories (73\%) reported values that were reasonably close to the target range. Two of the remaining three laboratories reported values that were significantly higher than the target range (150 to 200 times higher). These were the same laboratories that reported high values for the soy flour, indicating a potential interference in the analytical method or possibly a calibration error.

- One laboratory reported values that were 10 times lower than the target value for the soy flour and 35 times lower for the blueberries. This could be the result of ion suppression in the MS, as this laboratory reported using LC-MS with an external standard calibration approach. For accurate quantitation from matrix-based samples, the use of isotope dilution for internal standard calibration is critical.

- In general, the analytical approach used did not correlate with any trend in the data. In this case, variability in the data is more likely related to the combination of sample preparation, instrumental method, and calibration approach, as any method must be careful to account for interferences. A larger data set and more information from participants is necessary to draw any strong correlations between method and result.

\section{Technical Recommendations}

The following are recommendations based on results provided by the participants in this study.

- No analytical method was identified as being exceptionally good or problematic. When using LC-absorbance for a molecule like pantothenic acid without a chromophore, care must be taken to remove matrix interferences. The same is true when using LC-MS, as matrix components can cause ion suppression leading to results that are biased low unless an isotopically labeled analog is used for internal standard calibration. 
Table 7. Individual data table (NIST) for vitamin $\mathrm{B}_{5}$ (pantothenic acid) in foods.

\section{National Institute of Standards \& Technology}

Exercise I - October 2012 - Pantothenic Acid

\begin{tabular}{ccc} 
& Lab Code: & NIST \\
\hline Analyte & Sample & Units \\
\hline $\mathrm{B}_{5}$ & Soy Flour & $\mu \mathrm{g} / \mathrm{g}$ \\
$\mathrm{B}_{5}$ & Blueberries & $\mu \mathrm{g} / \mathrm{g}$ \\
\hline
\end{tabular}

\begin{tabular}{cccc}
\multicolumn{4}{c}{ 1. Your Results } \\
\hline $\mathrm{x}_{\mathrm{i}}$ & $\mathrm{s}_{\mathrm{i}}$ & $\mathrm{Z}_{\text {comm }}$ & $\mathrm{Z}_{\mathrm{NIST}}$ \\
\hline 10.7 & 0.10 & 2.3 & -0.4 \\
3.31 & 0.19 & -0.1 & 0.0
\end{tabular}

\begin{tabular}{ccc}
\multicolumn{3}{c}{ 2. Community Results } \\
\hline $\mathrm{N}$ & $\mathrm{x}^{*}$ & $\mathrm{~s}^{*}$ \\
\hline 13 & 18.6 & 15.7 \\
11 & 3.49 & 3.08 \\
\hline
\end{tabular}

\begin{tabular}{cc}
\multicolumn{2}{c}{ 3. Target } \\
\hline $\mathrm{x}_{\mathrm{NIST}}$ & $U_{95}$ \\
\hline 10.7 & 0.1 \\
3.31 & 0.19 \\
\hline
\end{tabular}

$\mathrm{x}_{\mathrm{i}} \quad$ Mean of reported values

$\mathrm{N}$ Number of quantitative

$\mathrm{x}_{\mathrm{NIST}}$

NIST-assessed value

$\mathrm{s}_{\mathrm{i}} \quad$ Standard deviation of reported values

$\mathrm{Z}_{\text {comm }} \quad$ Z-score with respect to community consensus

values reported

X* Robust mean of reported $\quad U_{95}$

values

s* Robust standard deviation

$Z_{\text {NIST }} \quad$ Z-score with respect to NIST value

$\pm 95 \%$ confidence interval about the assessed value or standard deviation ( $\mathrm{S}_{\mathrm{NIST}}$ ) 
Table 8. Data summary table for vitamin $B_{5}$ (pantothenic acid) in foods.

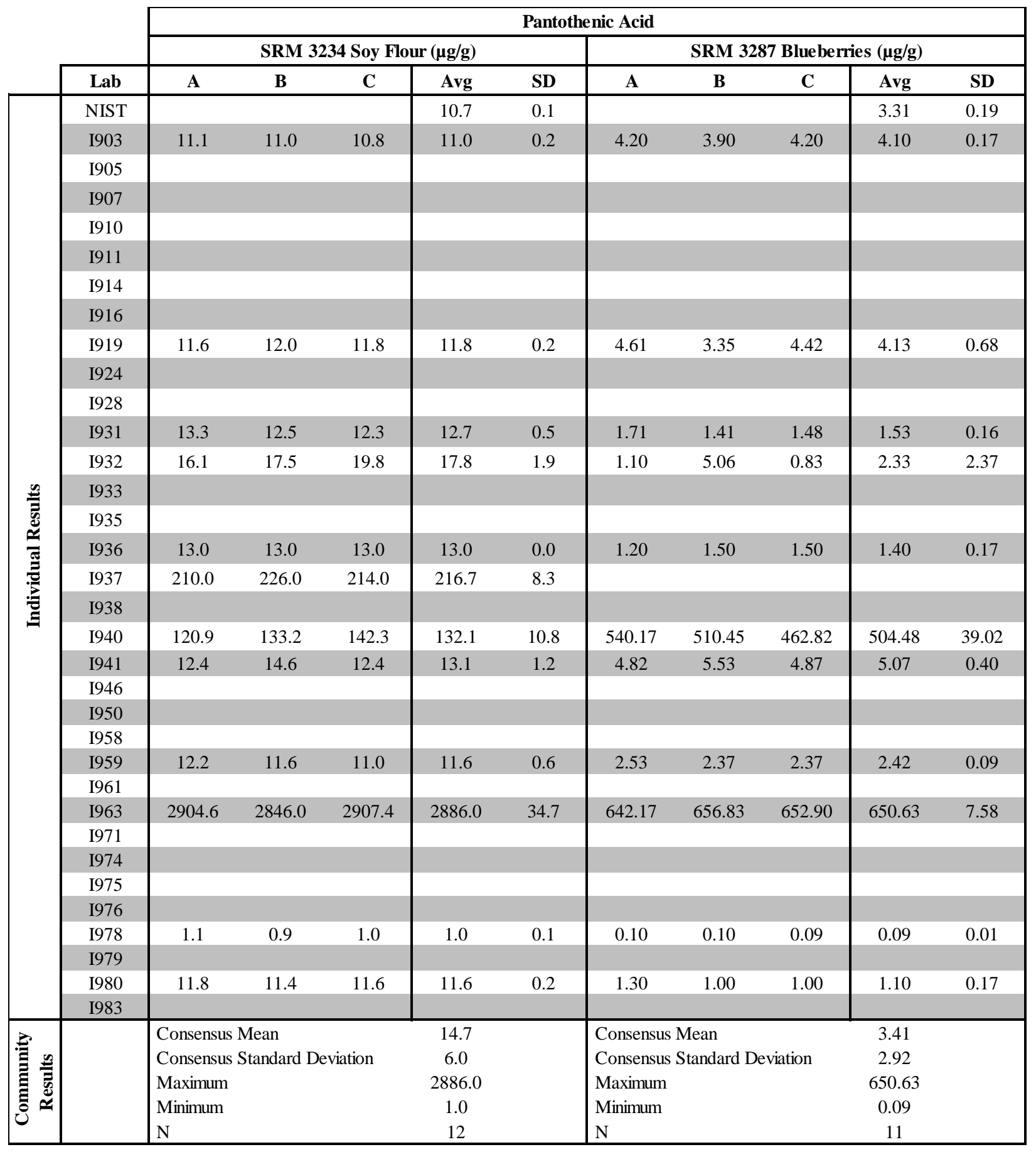




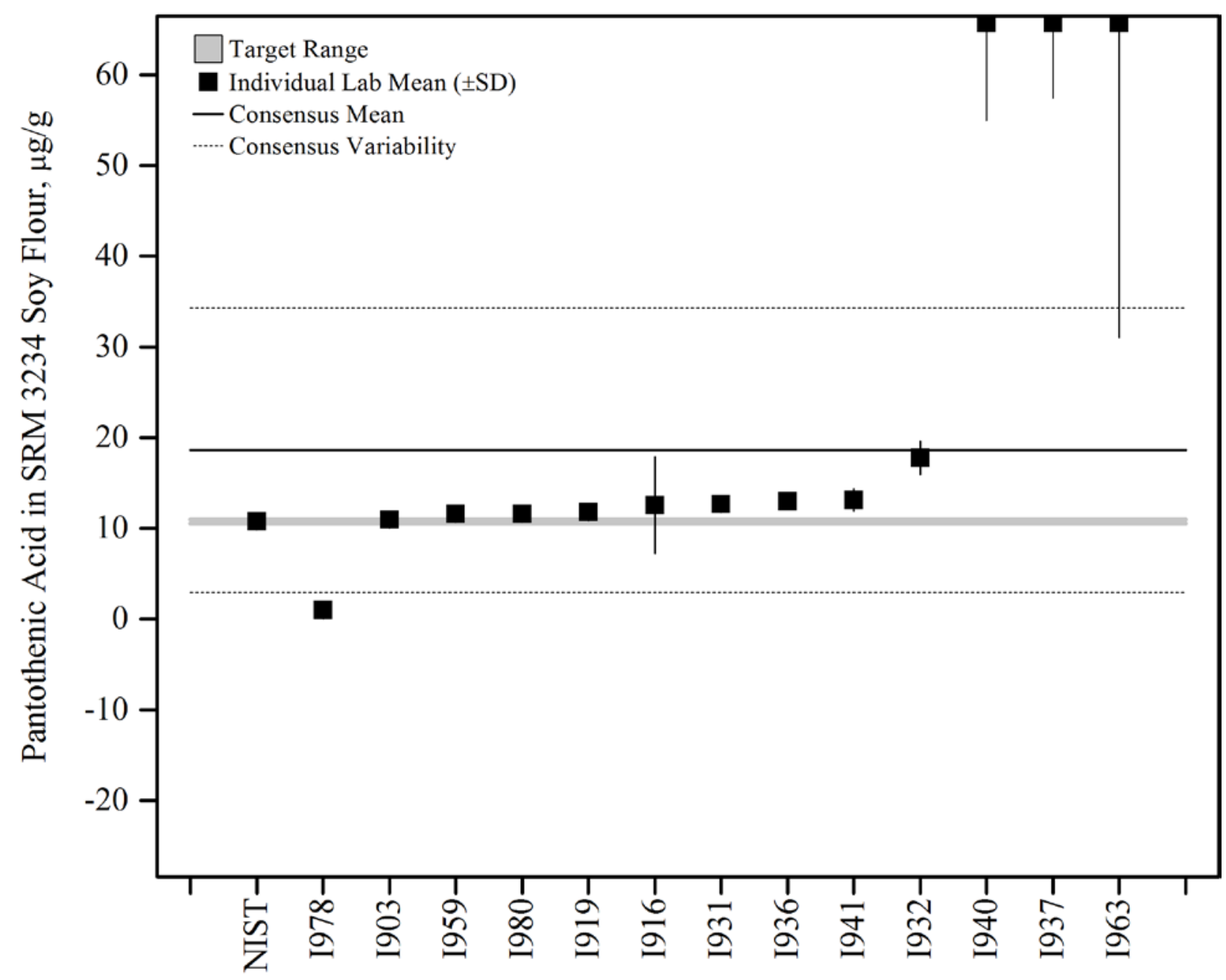

Figure 15. Vitamin $B_{5}$ (pantothenic acid) in SRM 3234 Soy Flour (data summary view). In this view, individual laboratory data are plotted with the individual laboratory standard deviation (error bars). The black solid line represents the consensus mean, and the black dotted lines represent the consensus variability calculated as one standard deviation about the consensus mean. The gray shaded region represents the target zone for "acceptable" performance, which encompasses the NIST certified value determined by ID-LC-MS/MS and external collaborating laboratories bounded by twice the uncertainty $\left(U_{95}\right)$. 


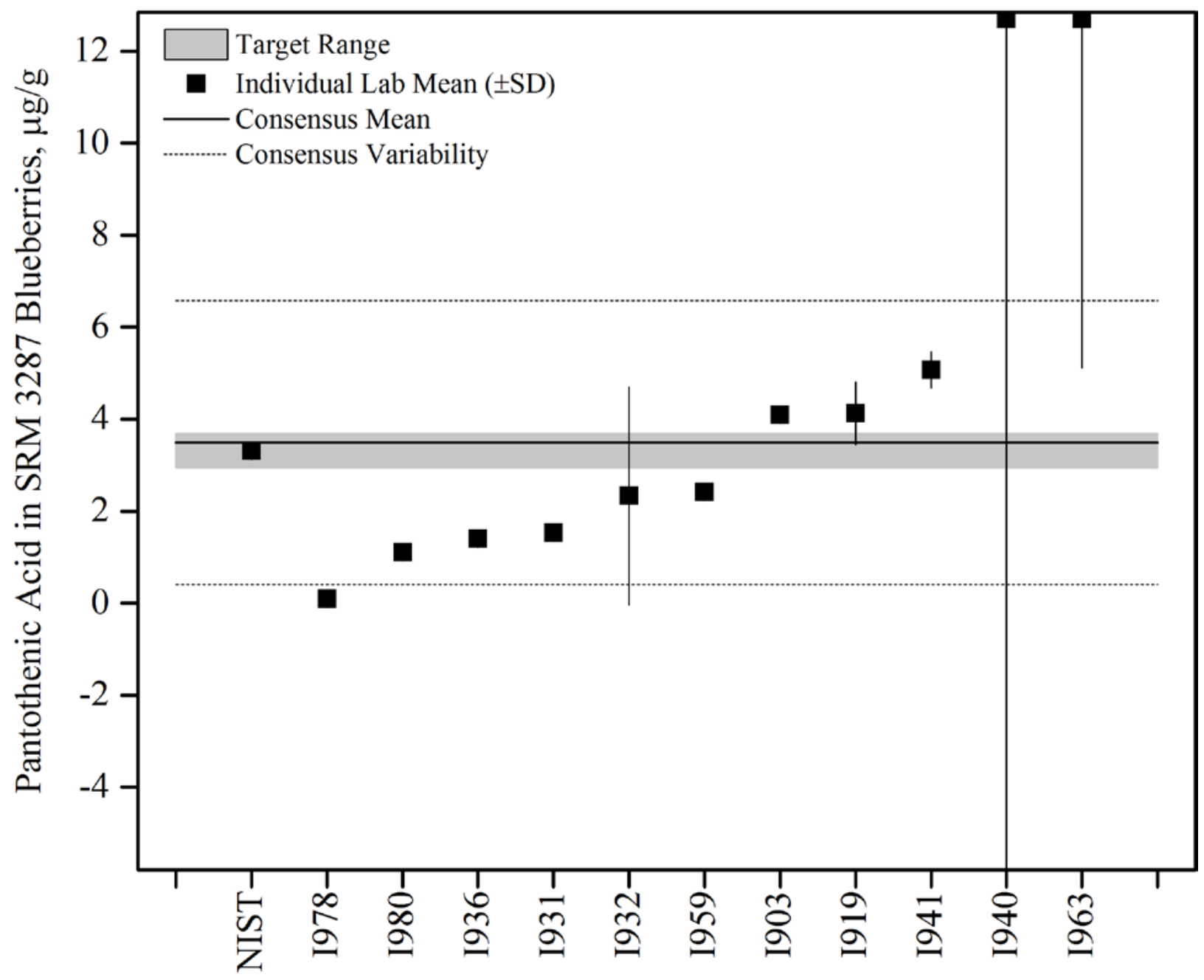

Figure 16. Vitamin $B_{5}$ (pantothenic acid) in SRM 3287 Blueberries (data summary view). In this view, individual laboratory data are plotted with the individual laboratory standard deviation (error bars). The black solid line represents the consensus mean, and the black dotted lines represent the consensus variability calculated as one standard deviation about the consensus mean. The gray shaded region represents the target zone for "acceptable” performance, which encompasses the NIST certified value determined by ID-LC-MS and external collaborating laboratories bounded by twice the uncertainty $\left(U_{95}\right)$. 


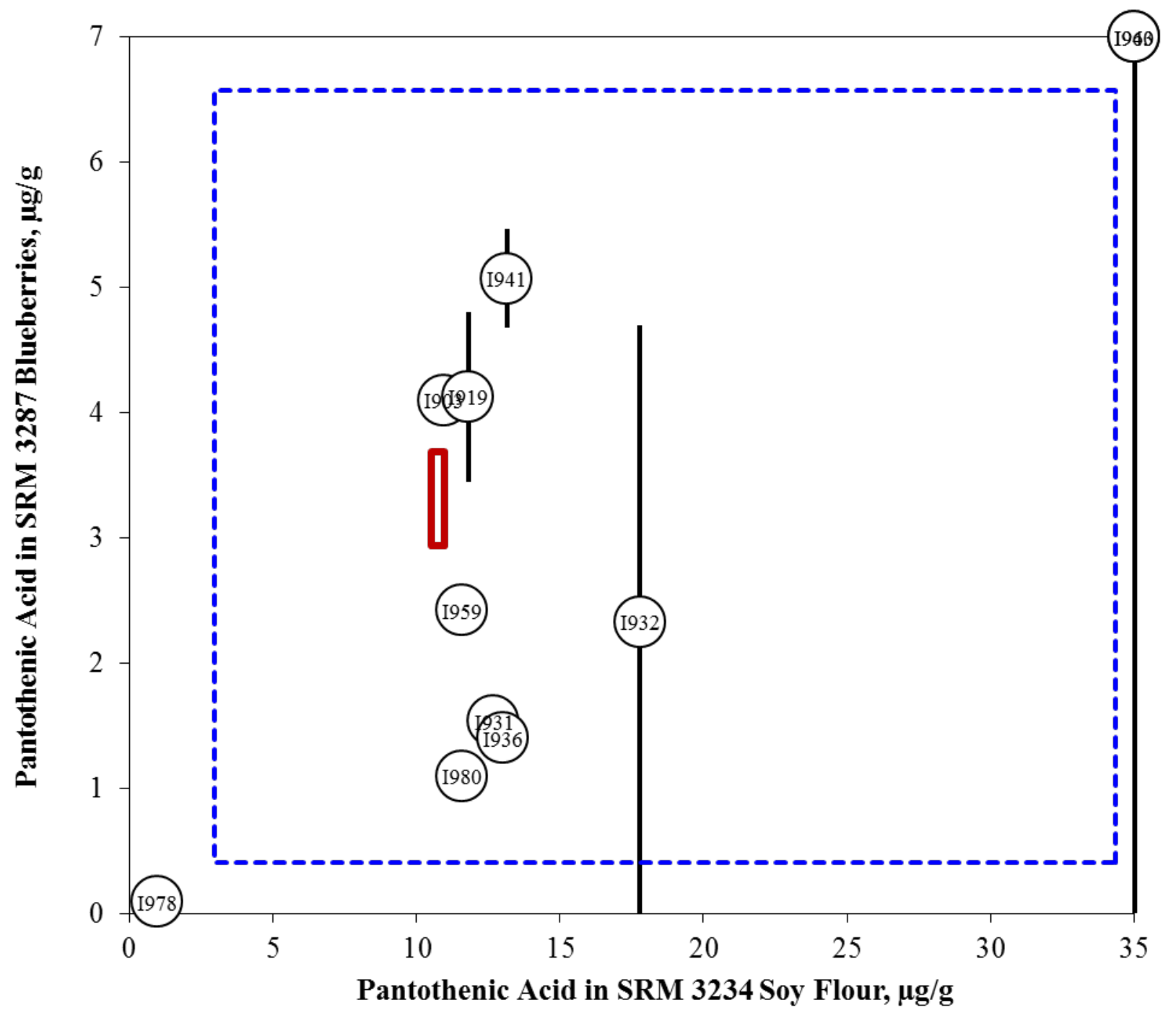

Figure 17. Vitamin $B_{5}$ (pantothenic acid) in SRM 3234 Soy Flour and SRM 3287 Blueberries (sample/sample comparison view). In this view, the individual laboratory results for one sample (SRM 3287 Blueberries) with a certified value for the analyte are compared to the results for a second sample (SRM 3234 Soy Flour). The error bars represent the individual laboratory standard deviation. The solid red lines represent the target zone for the control (x-axis) and the unknown sample (y-axis). The dotted blue box represents the consensus zone for the control (xaxis) and the unknown sample (y-axis). 


\section{VITAMIN A IN FOODS AND SUPPLEMENTS}

\section{$\underline{\text { Study Overview }}$}

In this study, participants were provided with SRM 3280 Multivitamin/Multielement Tablets and a whole egg powder. Participants were asked to use in-house analytical methods to determine the mass fractions of vitamin A (as retinol, retinyl acetate, and retinyl palmitate) in each of the matrices and report values on an as-received basis.

Sample Information

Multivitamin/Multielement Tablets. Participants were provided with one bottle containing 30 multivitamin/multielement tablets. Before use, participants were instructed to grind all tablets together, mix the resulting powder thoroughly, and use a sample size of at least $0.6 \mathrm{~g}$. Participants were asked to store the material at controlled room temperature, $10{ }^{\circ} \mathrm{C}$ to $30{ }^{\circ} \mathrm{C}$, prepare three samples, and report three values from the single bottle provided. Approximate analyte levels were not provided to participants prior to the study. The NIST reference values and uncertainties for vitamin A in SRM 3280 were determined by LC-MS following solvent extraction and are reported in the table below, both on a dry-mass basis and after correction for moisture of the material $(1.37 \%)$.

Egg powder. Participants were provided with one packet containing approximately $10 \mathrm{~g}$ of whole egg powder. The material is a free-flowing, fine powder prepared from USDA-inspected whole eggs. Before use, participants were instructed to mix thoroughly the contents of the packet and use a sample size of at least $1 \mathrm{~g}$. Participants were asked to store the material at controlled room temperature, $10^{\circ} \mathrm{C}$ to $30^{\circ} \mathrm{C}$, prepare three samples, and report three values from the single packet provided. Approximate analyte levels were not provided to participants prior to the study, and NIST-assessed values and uncertainties were not determined for the whole egg powder.

\section{Study Results}

- Thirty-seven laboratories enrolled in this exercise and received samples, and 19 laboratories reported results for at least one of the samples (51 \% participation).

- NIST target values are available for retinol equivalents and retinyl acetate in the multivitamin sample.

- The consensus mean for retinol and retinyl acetate were within the target range.

- The consensus ranges were acceptable for both compounds in the multivitamin sample (19\% for both compounds).

- Two laboratories reported values for retinyl palmitate in the multivitamin sample. The value from one laboratory appeared to be a conversion of the measured mass fraction of retinyl acetate to retinyl palmitate using the relative molecular masses of the compounds.

- $\quad$ NIST target values are not available for retinol in the egg powder sample. The consensus range for retinol in the egg powder was quite wide (83 \% RSD).

- Ten laboratories (53 \%) reported using saponification followed by extraction, while nine laboratories (47\%) reported using solvent extraction to prepare samples.

- A majority of laboratories (95\%) used LC-absorbance for analysis. One laboratory reported using spectrophotometry. 
- All laboratories reported using an external standard approach to calibration.

\section{Technical Recommendations}

The following recommendations are based on results provided by the participants in this study.

- It is important to determine that saponification methods are appropriate for a given sample. Conditions that are too extreme may result in degradation of the analyte of interest and conditions that are too gentle may not fully extract the analyte of interest. In future exercises, more survey information from participants will be collected about saponification to help aid in making recommendations.

- Always be certain that calibrants match the measured analyte (e.g., do not measure retinyl acetate with a retinol calibrant).

- Due to the nature of the calibrant materials, a spectrophotometric determination of calibrant concentration is essential for accurate measurements. 
Table 9. Individual data table (NIST) for vitamin A in foods and supplements.

\section{National Institute of Standards \& Technology}


Table 10. Data summary table for retinol in foods.

\begin{tabular}{|c|c|c|c|c|c|c|c|c|c|c|c|}
\hline & \multirow[b]{3}{*}{ Lab } & \multicolumn{10}{|c|}{ Retinol } \\
\hline & & \multicolumn{10}{|c|}{ SRM 3280 Multivitamin Tablet ( $\mu \mathrm{g} / \mathrm{g})$} \\
\hline & & A & B & $\mathrm{C}$ & Avg & SD & A & B & $\mathrm{C}$ & Avg & SD \\
\hline \multirow{38}{*}{ 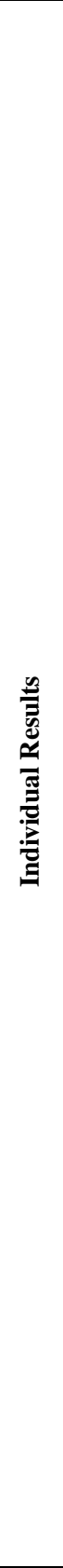 } & NIST & & & & 438 & 45 & & & & & \\
\hline & I901 & 387 & 410 & 409 & 402 & 13 & & & & & \\
\hline & I903 & 357 & 355 & 338 & 350 & 10 & 0.760 & 0.721 & 0.755 & 0.745 & 0.021 \\
\hline & I905 & & & & & & 0.600 & 0.500 & 0.400 & 0.500 & 0.100 \\
\hline & I907 & & & & & & & & & & \\
\hline & I910 & & & & & & & & & & \\
\hline & I911 & & & & & & & & & & \\
\hline & I914 & & & & & & & & & & \\
\hline & I915 & & & & & & & & & & \\
\hline & I916 & & & & & & & & & & \\
\hline & I919 & 408 & 444 & 406 & 419 & 21 & 0.622 & 0.655 & 0.675 & 0.651 & 0.027 \\
\hline & I922 & & & & & & & & & & \\
\hline & I924 & 425 & 408 & 385 & 406 & 20 & 2.100 & 1.770 & 1.250 & 1.707 & 0.429 \\
\hline & I928 & & & & & & & & & & \\
\hline & I929 & 560 & 543 & 524 & 542 & 18 & 25.000 & 28.000 & 23.000 & 25.333 & 2.517 \\
\hline & 1932 & & & & & & & & & & \\
\hline & I933 & & & & & & & & & & \\
\hline & I936 & & & & & & & & & & \\
\hline & I937 & 434 & 451 & 433 & 439 & 10 & & & & & \\
\hline & I938 & & & & & & & & & & \\
\hline & I940 & & & & & & & & & & \\
\hline & I946 & & & & & & & & & & \\
\hline & I949 & & & & & & & & & & \\
\hline & I950 & & & & & & & & & & \\
\hline & I958 & & & & & & & & & & \\
\hline & I959 & 774 & 774 & 726 & 758 & 28 & 3.200 & 3.000 & 3.200 & 3.133 & 0.115 \\
\hline & I961 & & & & & & & & & & \\
\hline & I963 & & & & & & & & & & \\
\hline & I965 & & & & & & & & & & \\
\hline & I971 & & & & & & & & & & \\
\hline & I974 & & & & & & & & & & \\
\hline & I975 & & & & & & & & & & \\
\hline & I977 & & & & & & & & & & \\
\hline & I978 & 400 & 304 & 409 & 371 & 58 & 2.874 & 2.001 & 2.351 & 2.409 & 0.439 \\
\hline & I979 & & & & & & & & & & \\
\hline & I980 & & & & & & 0.763 & 0.712 & 0.810 & 0.762 & 0.049 \\
\hline & I983 & & & & & & & & & & \\
\hline & I984 & & & & & & & & & & \\
\hline \multirow{5}{*}{ 攺 } & & \multirow{5}{*}{\multicolumn{3}{|c|}{$\begin{array}{l}\text { Consensus Mean } \\
\text { Consensus Standard Deviation } \\
\text { Maximum } \\
\text { Minimum } \\
\text { N }\end{array}$}} & 449 & & \multirow{5}{*}{\multicolumn{3}{|c|}{$\begin{array}{l}\text { Consensus Mean } \\
\text { Consensus Standarc } \\
\text { Maximum } \\
\text { Minimum } \\
\text { N }\end{array}$}} & 1.73 & \\
\hline & & & & & 94 & & & & & 1.47 & \\
\hline & & & & & 758 & & & & & 25.33 & \\
\hline & & & & & 350 & & & & & 0.50 & \\
\hline & & & & & 8 & & & & & 8 & \\
\hline
\end{tabular}


Table 11. Data summary table for retinyl acetate in foods.

\begin{tabular}{|c|c|c|c|c|c|c|c|c|c|c|c|}
\hline & \multirow[b]{3}{*}{ Lab } & \multicolumn{10}{|c|}{ Retinyl Acetate } \\
\hline & & \multicolumn{5}{|c|}{ SRM 3280 Multivitamin Tablet ( $\mu \mathrm{g} / \mathrm{g})$} & \multicolumn{5}{|c|}{ Whole Egg Powder ( $\mu \mathrm{g} / \mathrm{g})$} \\
\hline & & A & B & C & Avg & SD & A & B & C & Avg & SD \\
\hline \multirow{38}{*}{ 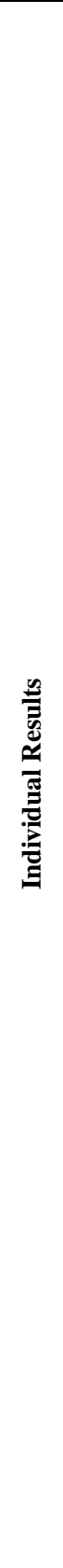 } & NIST & & & & 502 & 52 & & & & & \\
\hline & I901 & & & & & & & & & & \\
\hline & I903 & 472 & 468 & 445 & 462 & 15 & & & & & \\
\hline & $\mathrm{I} 905$ & 452 & 486 & 465 & 468 & 17 & & & & & \\
\hline & I907 & & & & & & & & & & \\
\hline & $\mathrm{I} 910$ & 490 & 508 & 512 & 503 & 12 & & & & & \\
\hline & $\mathrm{I} 911$ & & & & & & & & & & \\
\hline & $\mathrm{I} 914$ & & & & & & & & & & \\
\hline & $\mathrm{I} 915$ & & & & & & & & & & \\
\hline & I916 & & & & & & & & & & \\
\hline & $\mathrm{I} 919$ & 468 & 509 & 466 & 481 & 24 & & & & & \\
\hline & I922 & & & & & & & & & & \\
\hline & I924 & & & & & & & & & & \\
\hline & $\mathrm{I} 928$ & 399 & 413 & 404 & 405 & 7 & & & & & \\
\hline & I929 & & & & & & & & & & \\
\hline & $\mathrm{I} 932$ & 501 & 514 & 505 & 507 & 7 & & & & & \\
\hline & $\mathrm{I} 933$ & 1670 & 1700 & 1660 & 1677 & 21 & & & & & \\
\hline & I936 & & & & & & & & & & \\
\hline & I937 & & & & & & & & & & \\
\hline & I938 & & & & & & & & & & \\
\hline & $\mathrm{I} 940$ & 342 & 343 & 343 & 342 & 0 & 5.147 & 4.202 & 3.787 & 4.379 & 0.697 \\
\hline & I946 & & & & & & & & & & \\
\hline & I949 & 475 & 456 & 453 & 461 & 12 & & & & & \\
\hline & $\mathrm{I} 950$ & & & & & & & & & & \\
\hline & $\mathrm{I} 958$ & 700 & 789 & 760 & 750 & 45 & & & & & \\
\hline & I959 & & & & & & & & & & \\
\hline & I961 & & & & & & & & & & \\
\hline & I963 & 807 & 751 & 793 & 784 & 29 & & & & & \\
\hline & I965 & & & & & & & & & & \\
\hline & I971 & & & & & & & & & & \\
\hline & I974 & & & & & & & & & & \\
\hline & I975 & & & & & & & & & & \\
\hline & I977 & & & & & & & & & & \\
\hline & I978 & 459 & 349 & 469 & 426 & 67 & 3.296 & 2.294 & 2.696 & 2.762 & 0.504 \\
\hline & I979 & & & & & & & & & & \\
\hline & I980 & 384 & 384 & 357 & 375 & 16 & & & & & \\
\hline & I983 & & & & & & & & & & \\
\hline & I984 & & & & & & & & & & \\
\hline \multirow{5}{*}{ 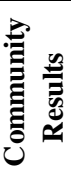 } & & \multicolumn{3}{|c|}{ Consensus Mean } & \multicolumn{2}{|l|}{493} & \multicolumn{2}{|c|}{ Consensus Mean } & & 3.57 & \\
\hline & & \multicolumn{3}{|c|}{ Consensus Standard Deviation } & \multicolumn{2}{|l|}{120} & \multicolumn{3}{|c|}{ Consensus Standard Deviation } & 1.30 & \\
\hline & & \multicolumn{3}{|c|}{ Maximum } & \multicolumn{2}{|l|}{1677} & \multicolumn{3}{|c|}{ Maximum } & 4.38 & \\
\hline & & \multicolumn{3}{|c|}{ Minimum } & \multicolumn{2}{|l|}{342} & \multicolumn{3}{|l|}{ Minimum } & 2.76 & \\
\hline & & \multicolumn{3}{|l|}{$\mathrm{N}$} & \multicolumn{2}{|l|}{13} & $\mathrm{~N}$ & & & 2 & \\
\hline
\end{tabular}


Table 12. Data summary table for retinyl palmitate in foods.

\begin{tabular}{|c|c|c|c|c|c|c|c|c|c|c|c|}
\hline & \multirow[b]{3}{*}{ Lab } & \multicolumn{10}{|c|}{ Retinyl Palmitate } \\
\hline & & \multicolumn{10}{|c|}{ SRM 3280 Multivitamin Tablet ( $\mu \mathrm{g} / \mathrm{g})$} \\
\hline & & A & B & $\mathbf{C}$ & Avg & SD & A & $\mathbf{B}$ & $\mathrm{C}$ & Avg & SD \\
\hline \multirow{38}{*}{ 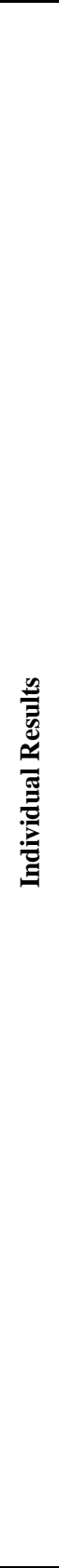 } & NIST & & & & & & & & & & \\
\hline & I901 & & & & & & & & & & \\
\hline & I903 & & & & & & & & & & \\
\hline & I905 & & & & & & & & & & \\
\hline & I907 & & & & & & & & & & \\
\hline & I910 & & & & & & & & & & \\
\hline & I911 & & & & & & & & & & \\
\hline & I914 & & & & & & & & & & \\
\hline & I915 & & & & & & & & & & \\
\hline & I916 & & & & & & & & & & \\
\hline & I919 & & & & & & & & & & \\
\hline & I922 & & & & & & & & & & \\
\hline & I924 & & & & & & & & & & \\
\hline & I928 & & & & & & & & & & \\
\hline & I929 & & & & & & & & & & \\
\hline & I932 & & & & & & & & & & \\
\hline & I933 & & & & & & & & & & \\
\hline & I936 & & & & & & & & & & \\
\hline & I937 & & & & & & & & & & \\
\hline & I938 & & & & & & & & & & \\
\hline & I940 & & & & & & & & & & \\
\hline & I946 & & & & & & & & & & \\
\hline & I949 & & & & & & & & & & \\
\hline & I950 & & & & & & & & & & \\
\hline & I958 & & & & & & & & & & \\
\hline & I959 & & & & & & & & & & \\
\hline & I961 & & & & & & & & & & \\
\hline & I963 & 85 & 144 & 78 & 102 & 36 & & & & & \\
\hline & I965 & & & & & & & & & & \\
\hline & I971 & & & & & & & & & & \\
\hline & I974 & & & & & & & & & & \\
\hline & I975 & & & & & & & & & & \\
\hline & I977 & & & & & & & & & & \\
\hline & I978 & 734 & 558 & 750 & 681 & 107 & 5.27 & 3.67 & 4.31 & 4.42 & 0.81 \\
\hline & I979 & & & & & & & & & & \\
\hline & I980 & & & & & & & & & & \\
\hline & I983 & & & & & & & & & & \\
\hline & I984 & & & & & & & & & & \\
\hline 总 & & \multicolumn{3}{|c|}{$\begin{array}{l}\text { Consensus Mean } \\
\text { Consensus Standard Deviation } \\
\text { Maximum } \\
\text { Minimum } \\
\text { N }\end{array}$} & $\begin{array}{c}392 \\
464 \\
681 \\
102 \\
2\end{array}$ & & \multicolumn{3}{|c|}{$\begin{array}{l}\text { Consensus Mean } \\
\text { Consensus Standard Deviation } \\
\text { Maximum } \\
\text { Minimum } \\
\mathrm{N} \\
\end{array}$} & $\begin{array}{c}4.42 \\
4.42 \\
1\end{array}$ & \\
\hline
\end{tabular}




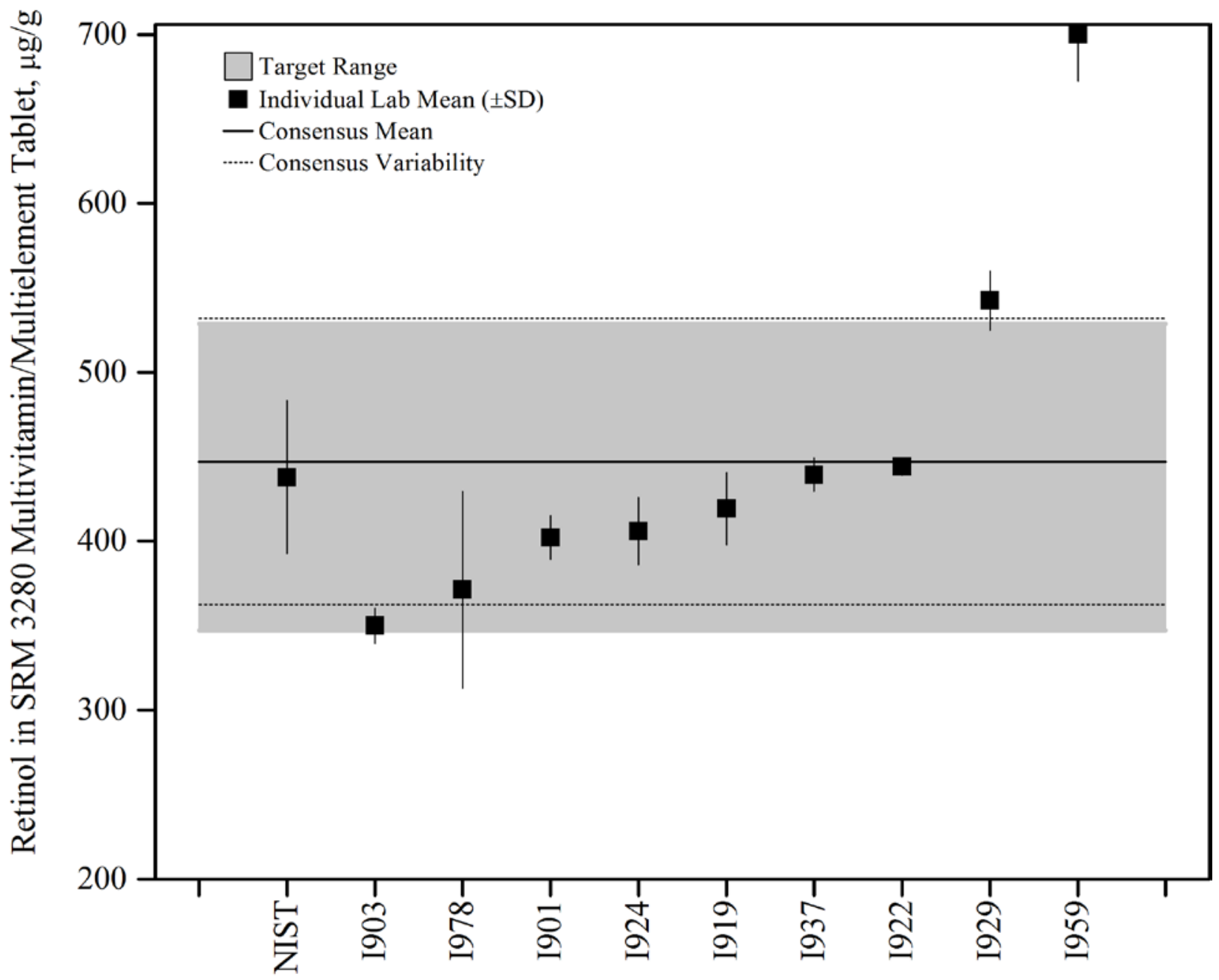

Figure 18. Retinol in SRM 3280 Multivitamin/Multielement Tablet (data summary view). In this view, individual laboratory data are plotted with the individual laboratory standard deviation (error bars). Data points that are unfilled represent laboratories that only reported a single value for that analyte and therefore were not included in the consensus mean. The black solid line represents the consensus mean, and the black dotted lines represent the consensus variability calculated as one standard deviation about the consensus mean. The gray shaded region represents the target zone for "acceptable” performance, which encompasses the NIST reference value determined by LC-MS (measured as retinyl acetate, expressed as retinol equivalents) bounded by twice the uncertainty $\left(U_{95}\right)$. 


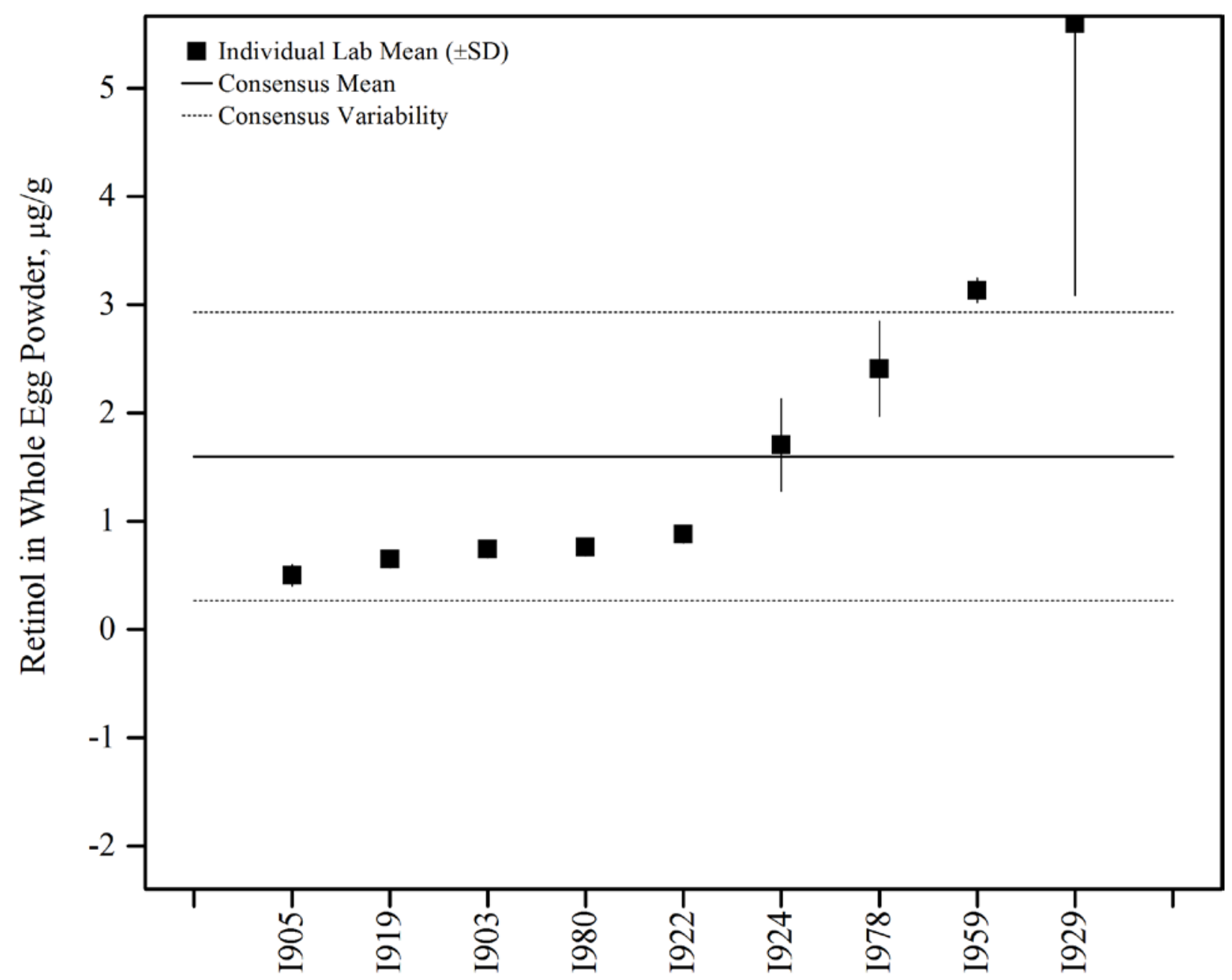

Figure 19. Retinol in whole egg powder (data summary view). In this view, individual laboratory data are plotted with the individual laboratory standard deviation (error bars). The black solid line represents the consensus mean, and the black dotted lines represent the consensus variability calculated as one standard deviation about the consensus mean. 


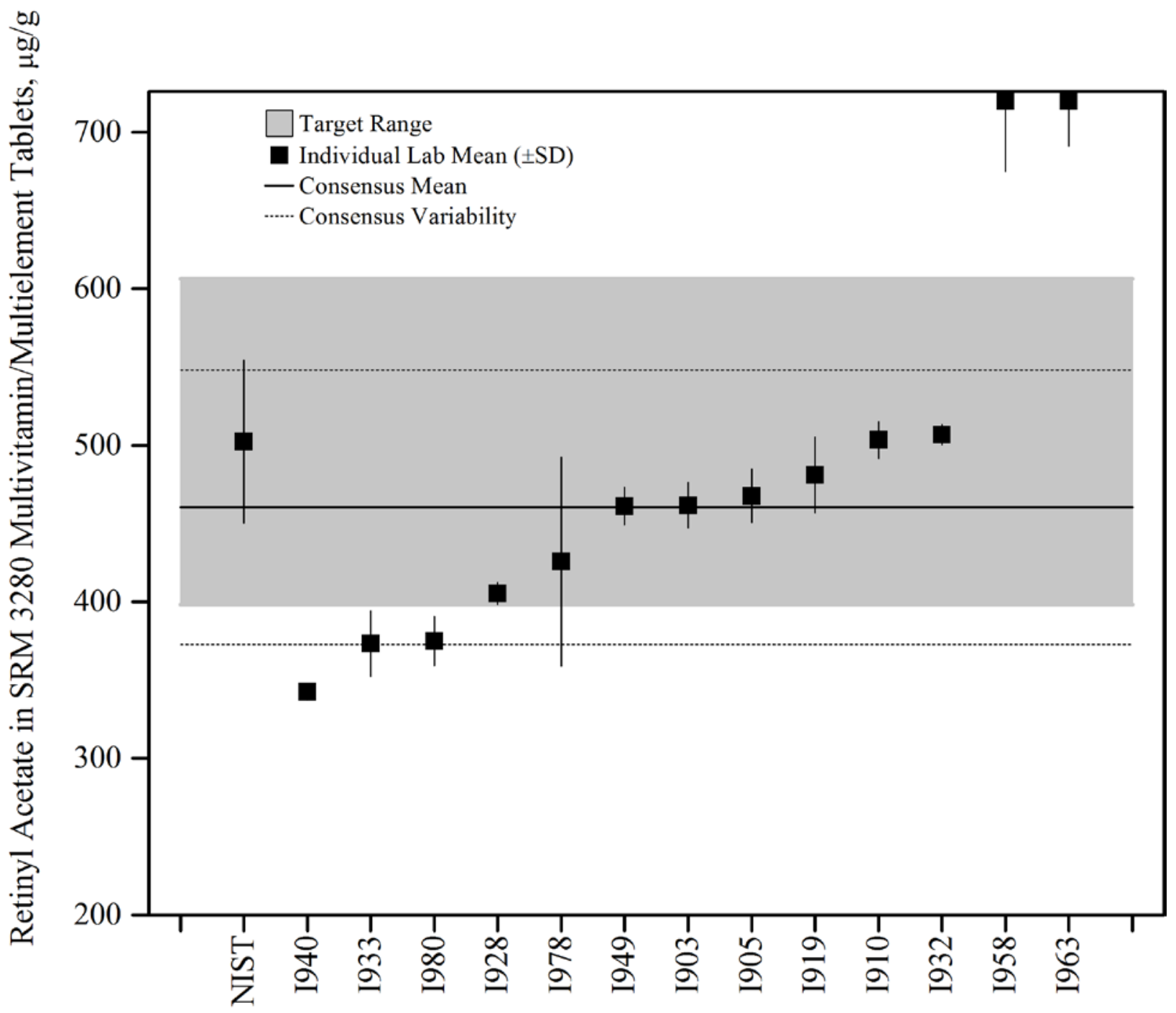

Figure 20. Retinyl acetate in SRM 3280 Multivitamin/Multielement Tablets (data summary view). In this view, individual laboratory data are plotted with the individual laboratory standard deviation (error bars). The black solid line represents the consensus mean, and the black dotted lines represent the consensus variability calculated as one standard deviation about the consensus mean. The gray shaded region represents the target zone for "acceptable" performance, which encompasses the NIST reference value determined by LC-MS bounded by twice the uncertainty $\left(U_{95}\right)$. 


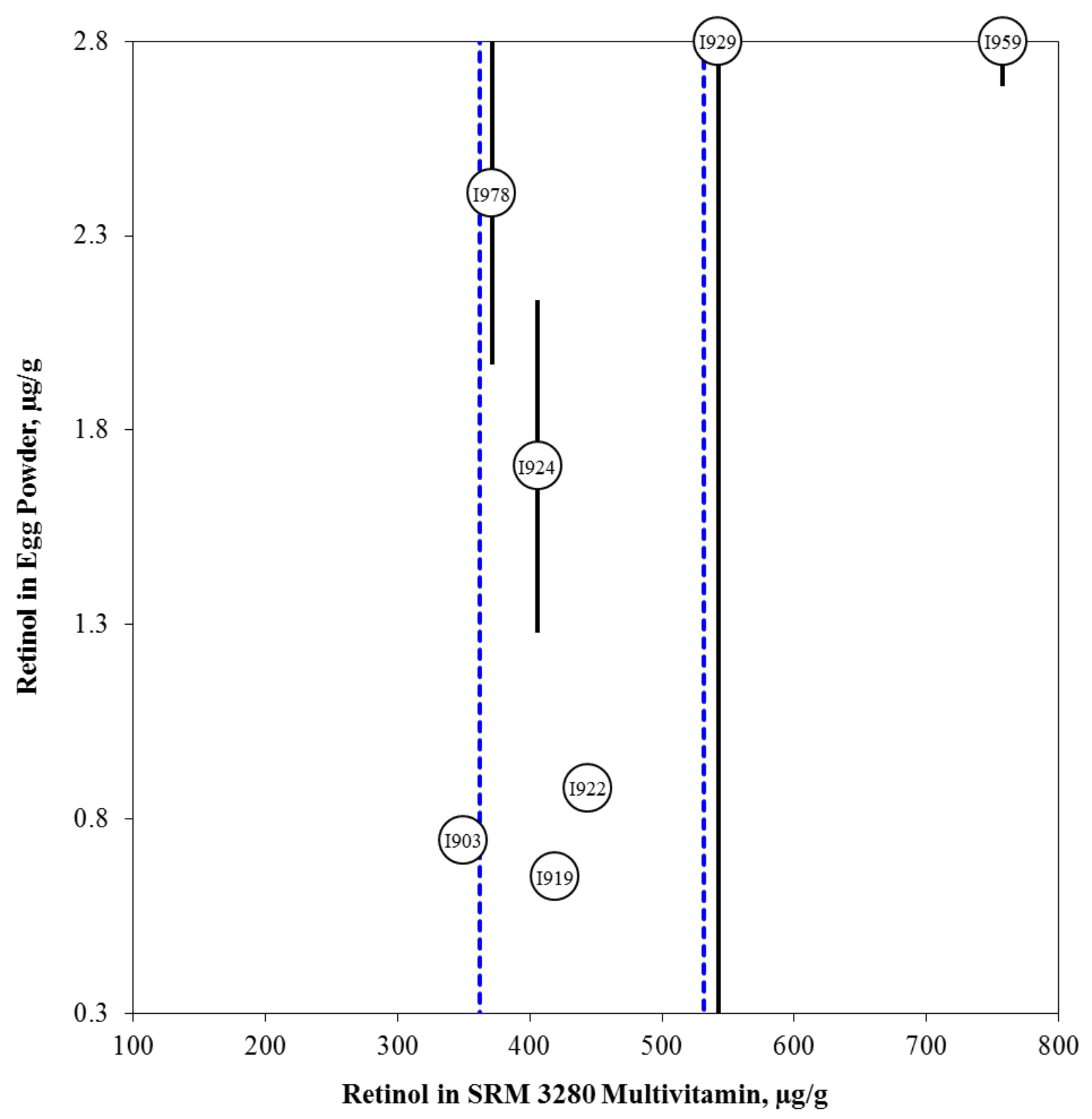

Figure 21. Retinol in whole egg powder and SRM 3280 Multivitamin/Multielement Tablets (sample/control comparison view). In this view, the individual laboratory results for the control (SRM 3280 Multivitamin/Multielement Tablets) with a reference value for the analyte are compared to the results for an unknown (whole egg powder). The error bars represent the individual laboratory standard deviation. The dotted blue box represents the consensus zone for the control (x-axis) and the unknown sample (y-axis). 


\section{CATECHINS IN GREEN TEA}

\section{Study Overview}

In this study, participants were provided with two NIST SRMs, SRM 3255 Camellia sinensis (Green Tea) Extract and SRM 3254 Camellia sinensis (Green Tea) Leaves. Participants were asked to use in-house analytical methods to determine the mass fractions of seven catechins (catechin, epicatechin, epicatechin gallate, epigallocatechin, epigallocatechin gallate, gallocatechin, and gallocatechin gallate), as well as the total amount of catechins, in each of the matrices and report values on an as-received basis.

\section{Sample Information}

Green tea extract. Participants were provided with three packets, each containing approximately $1 \mathrm{~g}$ of an extract of green tea extract. The spray-dried extract of green tea leaves was heat-sealed inside nitrogen-flushed $0.1 \mathrm{~mm}$ (4 mil) polyethylene bags, which were then sealed inside aluminized plastic bags with two packets of silica gel. Before use, participants were instructed to thoroughly mix the contents of each packet and use a sample size of at least $50 \mathrm{mg}$. Participants were asked to store the extract at controlled room temperature, $10^{\circ} \mathrm{C}$ to $30{ }^{\circ} \mathrm{C}$, and report a single value from each packet. Approximate analyte levels were not provided to participants prior to the study. The NIST certified values in SRM 3255 were determined by LC-UV, LC-MS, and data from external collaborating laboratories. The certified values and their associated uncertainties, corrected for the moisture content of the material (3.13\%), are provided on an asreceived basis in the table below.

Green tea leaves. Participants were provided with three packets, each containing approximately $3 \mathrm{~g}$ of green tea leaves. The ground green tea leaves were heat-sealed inside nitrogen-flushed 0.1 mm (4 mil) polyethylene bags, which were then sealed inside aluminized plastic bags with 2 packets of silica gel. Before use, participants were instructed to thoroughly mix the contents of the packet and use a sample size of at least $0.4 \mathrm{~g}$. Participants were asked to store the material at controlled room temperature, $10^{\circ} \mathrm{C}$ to $30^{\circ} \mathrm{C}$, and report a single value from each packet. Approximate analyte levels were not provided to participants prior to the study. The NIST certified values in SRM 3254 were determined by LC-UV, LC-MS, and data from external collaborating laboratories. The certified values and their associated uncertainties, corrected for the moisture content of the material (5.19\%), are provided on an as-received basis in the table below.

\author{
Analyte \\ Catechin \\ Epicatechin \\ Epicatechin Gallate \\ Epigallocatechin \\ Epigallocatechin Gallate \\ Gallocatechin \\ Gallocatechin Gallate \\ Total Catechins
}

\begin{tabular}{|c|c|c|}
\hline $\begin{array}{c}\text { Certified I } \\
\text { in SRM } \\
\text { (as-rece }\end{array}$ & 255 & $\begin{array}{l}\text { Fraction } \\
\text { (mg/g) } \\
\text { basis) }\end{array}$ \\
\hline 8.88 & \pm & 0.90 \\
\hline 45.8 & \pm & 6.5 \\
\hline 97.2 & \pm & 7.6 \\
\hline 79.2 & \pm & 6.3 \\
\hline 409 & \pm & 18 \\
\hline 21.3 & \pm & 1.6 \\
\hline 37.8 & \pm & 1.9 \\
\hline 699 & \pm & 22 \\
\hline
\end{tabular}




\section{Study Results}

- Forty-nine laboratories enrolled in this exercise and received samples, and twenty-eight laboratories reported results (57 \% participation).

- The consensus means for catechin, epicatechin, epicatechin gallate, and epigallocatechin gallate in the extract were within the target range, with acceptable consensus ranges (9\% to $24 \%$ RSD).

- The consensus mean for epigallocatechin was slightly below the target range, while the consensus means for gallocatechin and gallocatechin gallate were slightly above the target range. The consensus ranges were quite wide for all three (25\% to $72 \%$ RSD).

- The consensus means for catechin, epicatechin, epicatechin gallate, epigallocatechin, and epigallocatechin gallate in the ground tea leaves were within the target range, with acceptable consensus ranges for epicatechin, epicatechin gallate, and epigallocatechin gallate (21 \% to $23 \%$ RSD). The consensus ranges for catechin and epigallocatechin were significantly wider (67 \% RSD and $59 \%$ RSD, respectively).

- The consensus means for gallocatechin and gallocatechin gallate were higher than the target range with wide consensus ranges (65 \% to $112 \%$ RSD).

- The consensus means for total catechins in both the extract and the leaves were within the target range, with acceptable consensus ranges (11 \% and $24 \%$ RSD, respectively).

- Laboratories that reported low values typically reported low values for all of the analytes in both matrices. The same is true for those laboratories reporting high values.

- Twenty-seven (96 \%) of the laboratories reported using solvent extraction followed by LC-absorbance with external standard calibration. One laboratory reported using solvent extraction with LC-MS and external standard calibration.

- This study was previously conducted in Exercise E of the DSQAP (2010). The results for this study are significantly improved, with twice as many laboratories participating and more consistent results for nearly all of the individual catechins.

\section{Technical Recommendations}

The following recommendations are based on results provided by the participants in this study.

- Some laboratories (those reporting all high or low values) may have a calibration or sample preparation issue. Calibrant materials should be subjected to the same preparation procedure as the samples (derivatization, hydrolysis, etc.), and individual calibration standards should be used for each compound to improve accuracy.

- When sample preparation is extensive, an internal standard approach may be required to improve accuracy and precision.

- If an internal standard approach is used, it is best to add the internal standard at the earliest possible point (i.e. prior to extraction, saponification, and/or derivatization). 
Table 13. Individual data table (NIST) for catechins in green tea.

\section{National Institute of Standards \& Technology}

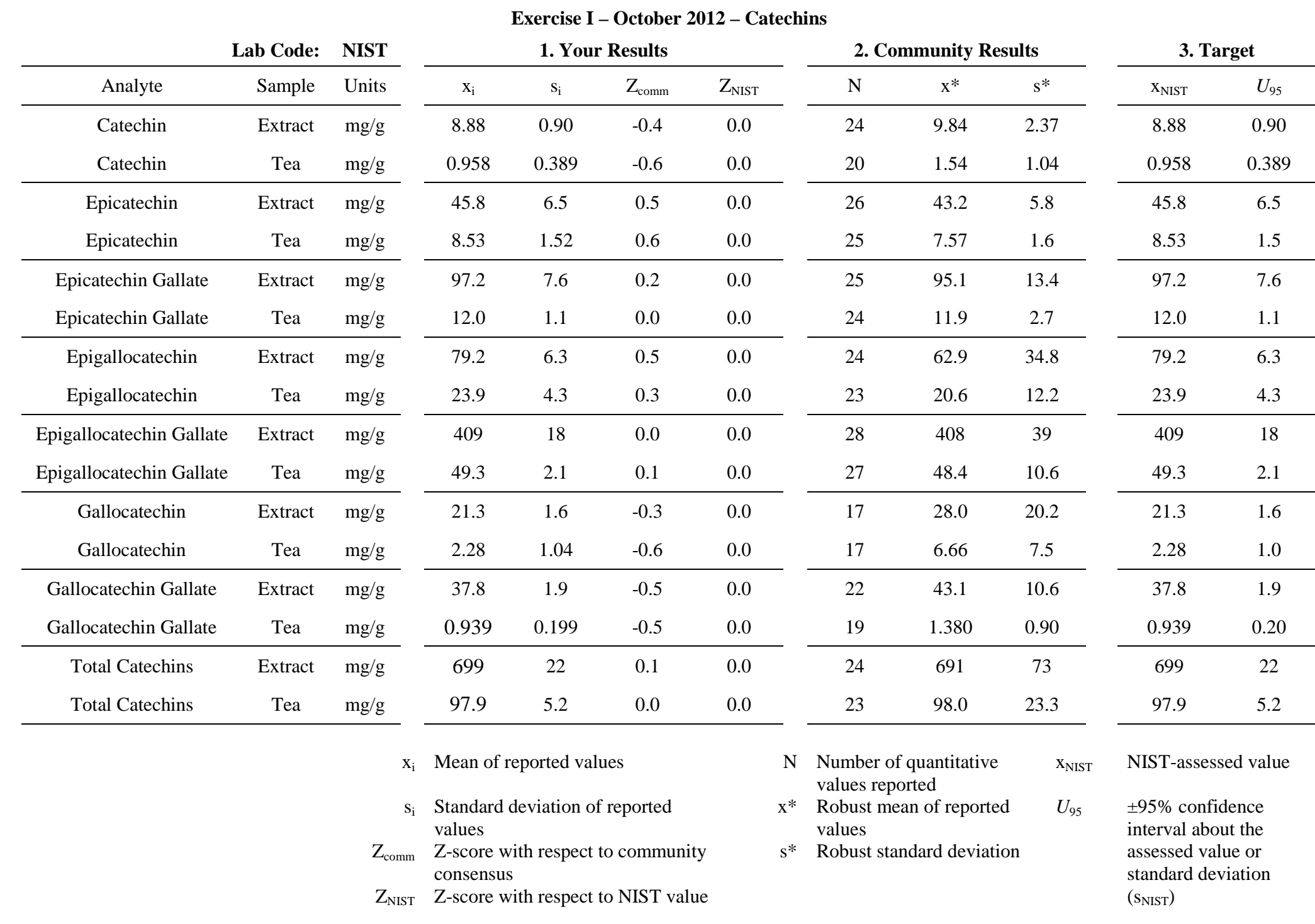


Table 14. Data summary table for catechin in green tea.

\begin{tabular}{|c|c|c|c|c|c|c|c|c|c|c|c|}
\hline & \multirow[b]{3}{*}{ Lab } & \multicolumn{10}{|c|}{ Catechin } \\
\hline & & \multicolumn{5}{|c|}{ SRM 3255 Green Tea Extract (mg/g) } & \multicolumn{5}{|c|}{ SRM 3254 Green Tea (mg/g) } \\
\hline & & A & B & C & Avg & SD & A & B & C & Avg & SD \\
\hline \multirow{50}{*}{ 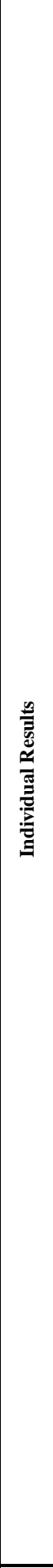 } & NIST & & & & 8.88 & 0.90 & & & & 0.958 & 0.389 \\
\hline & I901 & & & & & & & & & & \\
\hline & I902 & 11.80 & 11.50 & 11.70 & 11.67 & 0.15 & 0.970 & 1.140 & 1.000 & 1.037 & 0.091 \\
\hline & I903 & 8.48 & 8.42 & 8.61 & 8.50 & 0.10 & 0.886 & 0.905 & 0.911 & 0.901 & 0.013 \\
\hline & I904 & & & & & & & & & & \\
\hline & I905 & 10.30 & 10.20 & 10.20 & 10.23 & 0.06 & 1.700 & 1.700 & 1.700 & 1.700 & 0.000 \\
\hline & I906 & & & & & & & & & & \\
\hline & I907 & & & & & & & & & & \\
\hline & I909 & 4.71 & 5.10 & 5.22 & 5.01 & 0.27 & 0.201 & 0.205 & 0.188 & 0.198 & 0.009 \\
\hline & I911 & & & & & & & & & & \\
\hline & I912 & 12.40 & 12.40 & 12.20 & 12.33 & 0.12 & 2.170 & 2.130 & 2.110 & 2.137 & 0.031 \\
\hline & I913 & 0.23 & 0.18 & 0.25 & 0.22 & 0.04 & & & & & \\
\hline & I914 & & & & & & & & & & \\
\hline & I916 & & & & & & & & & & \\
\hline & I918 & 10.09 & 10.45 & 10.43 & 10.32 & 0.20 & 1.159 & 1.037 & 0.968 & 1.055 & 0.097 \\
\hline & I921 & & & & & & & & & & \\
\hline & I922 & & & & & & & & & & \\
\hline & I923 & 8.73 & 8.81 & 8.77 & 8.77 & 0.04 & 0.780 & 0.870 & 0.770 & 0.807 & 0.055 \\
\hline & I926 & 12.20 & 12.30 & 12.60 & 12.37 & 0.21 & & & & & \\
\hline & I927 & 12.60 & 12.90 & 12.73 & 12.75 & 0.15 & 0.894 & 1.154 & 1.262 & 1.104 & 0.189 \\
\hline & I928 & & & & & & & & & & \\
\hline & I930 & & & & & & & & & & \\
\hline & I933 & 141.63 & 141.30 & 140.54 & 141.16 & 0.56 & 62.380 & 62.900 & 62.740 & 62.673 & 0.266 \\
\hline & I934 & 10.20 & 10.09 & 10.28 & 10.19 & 0.10 & 1.964 & 2.676 & 15.283 & 6.641 & 7.493 \\
\hline & I938 & & & & & & & & & & \\
\hline & I939 & 10.23 & 10.12 & 10.07 & 10.14 & 0.08 & 2.816 & 2.772 & 2.863 & 2.817 & 0.045 \\
\hline & I940 & & & & & & & & & & \\
\hline & I943 & 10.76 & 10.88 & 11.06 & 10.90 & 0.15 & 1.962 & 1.857 & 1.849 & 1.890 & 0.063 \\
\hline & I944 & & & & & & & & & & \\
\hline & I946 & 10.70 & 10.59 & 10.61 & 10.63 & 0.06 & 0.907 & 0.907 & 0.914 & 0.909 & 0.004 \\
\hline & I947 & & & & & & & & & & \\
\hline & I950 & & & & & & & & & & \\
\hline & I952 & 9.22 & 9.32 & 9.72 & 9.42 & 0.26 & 1.240 & 1.180 & 1.190 & 1.203 & 0.032 \\
\hline & I953 & 111.63 & 113.17 & 111.07 & 111.96 & 1.09 & 59.450 & 60.930 & 62.330 & 60.903 & 1.440 \\
\hline & I954 & 4.83 & 4.73 & 4.75 & 4.77 & 0.05 & 0.617 & 0.602 & 0.624 & 0.614 & 0.011 \\
\hline & I956 & & & & & & & & & & \\
\hline & I957 & & & & & & & & & & \\
\hline & I958 & & & & & & & & & & \\
\hline & I963 & 10.18 & 10.23 & 10.12 & 10.18 & 0.06 & 2.302 & 2.326 & 2.260 & 2.296 & 0.033 \\
\hline & I964 & 7.70 & 7.63 & 7.76 & 7.70 & 0.07 & 0.530 & 0.500 & 0.470 & 0.500 & 0.030 \\
\hline & I965 & & & & & & & & & & \\
\hline & I966 & 8.40 & 7.80 & 8.00 & 8.07 & 0.31 & 1.000 & 1.200 & 1.100 & 1.100 & 0.100 \\
\hline & I968 & & & & & & & & & & \\
\hline & I969 & & & & & & & & & & \\
\hline & I970 & & & & & & & & & & \\
\hline & I976 & & & & & & & & & & \\
\hline & I979 & & & & & & & & & & \\
\hline & I982 & 8.03 & 7.00 & 7.63 & 7.55 & 0.52 & & 0.100 & & 0.100 & \\
\hline & I984 & & & & & & & & & & \\
\hline & I985 & 9.86 & 10.01 & 10.02 & 9.97 & 0.09 & 3.031 & 2.998 & 3.048 & 3.026 & 0.025 \\
\hline \multirow{5}{*}{ 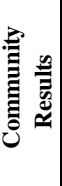 } & & \multicolumn{3}{|c|}{ Consensus Mean } & \multicolumn{2}{|l|}{9.87} & \multicolumn{2}{|c|}{ Consensus Mean } & & 1.602 & \\
\hline & & \multicolumn{3}{|c|}{ Consensus Standard Deviation } & \multicolumn{2}{|l|}{2.68} & \multicolumn{3}{|c|}{ Consensus Standard Deviation } & 1.229 & \\
\hline & & \multicolumn{3}{|l|}{ Maximum } & \multicolumn{2}{|l|}{111.96} & Maximum & & & 60.903 & \\
\hline & & Minimum & & & 4.77 & & Minimum & & & 0.100 & \\
\hline & & $\mathrm{N}$ & & & 11 & & $\mathrm{~N}$ & & & 10 & \\
\hline
\end{tabular}


Table 15. Data summary table for epicatechin in green tea.

\begin{tabular}{|c|c|c|c|c|c|c|c|c|c|c|c|}
\hline & \multirow[b]{3}{*}{ Lab } & \multicolumn{10}{|c|}{ Epicatechin } \\
\hline & & \multicolumn{5}{|c|}{ SRM 3255 Green Tea Extract (mg/g) } & \multicolumn{5}{|c|}{ SRM 3254 Green Tea (mg/g) } \\
\hline & & $\mathbf{A}$ & B & C & Avg & SD & $\mathbf{A}$ & B & C & Avg & SD \\
\hline \multirow{50}{*}{ 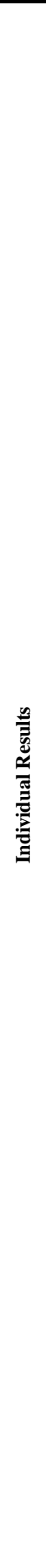 } & NIST & & & & 45.8 & 6.5 & & & & 8.53 & 1.52 \\
\hline & I901 & & & & & & & & & & \\
\hline & I902 & 179.0 & 87.8 & 179.0 & 148.6 & 52.7 & 77.50 & 77.70 & 77.40 & 77.53 & 0.15 \\
\hline & I903 & 45.3 & 45.2 & 45.5 & 45.3 & 0.2 & 8.60 & 8.36 & 8.35 & 8.44 & 0.14 \\
\hline & I904 & & & & & & & & & & \\
\hline & I905 & 47.4 & 47.3 & 47.2 & 47.3 & 0.1 & 8.40 & 8.50 & 8.30 & 8.40 & 0.10 \\
\hline & I906 & & & & & & & & & & \\
\hline & I907 & & & & & & & & & & \\
\hline & I909 & 34.3 & 37.4 & 41.5 & 37.7 & 3.6 & 7.72 & 7.40 & 7.42 & 7.51 & 0.18 \\
\hline & I911 & & & & & & & & & & \\
\hline & I912 & 45.5 & 45.6 & 45.1 & 45.4 & 0.3 & 8.21 & 7.73 & 7.66 & 7.87 & 0.30 \\
\hline & I913 & 2.5 & 2.0 & 2.7 & 2.4 & 0.4 & 0.20 & 0.18 & 0.20 & 0.19 & 0.01 \\
\hline & I914 & & & & & & & & & & \\
\hline & I916 & & & & & & & & & & \\
\hline & I918 & 47.1 & 46.5 & 46.1 & 46.6 & 0.5 & 8.05 & 7.74 & 7.37 & 7.72 & 0.34 \\
\hline & I921 & & & & & & & & & & \\
\hline & I922 & & & & & & & & & & \\
\hline & I923 & 45.4 & 47.9 & 48.3 & 47.2 & 1.6 & 5.86 & 6.12 & 5.45 & 5.81 & 0.34 \\
\hline & I926 & 33.6 & 33.9 & 34.1 & 33.9 & 0.3 & & & & & \\
\hline & I927 & 48.3 & 48.8 & 48.3 & 48.5 & 0.3 & 6.01 & 8.24 & 8.11 & 7.45 & 1.25 \\
\hline & I928 & & & & & & & & & & \\
\hline & I930 & & & & & & & & & & \\
\hline & I933 & 46.2 & 45.6 & 45.5 & 45.8 & 0.4 & 6.43 & 6.69 & 6.54 & 6.55 & 0.13 \\
\hline & I934 & 43.5 & 44.3 & 43.8 & 43.9 & 0.4 & 6.24 & 6.07 & 6.79 & 6.37 & 0.37 \\
\hline & I938 & & & & & & & & & & \\
\hline & I939 & 42.6 & 42.4 & 42.3 & 42.4 & 0.2 & 7.35 & 7.27 & 7.26 & 7.29 & 0.05 \\
\hline & I940 & & & & & & & & & & \\
\hline & I943 & 43.2 & 43.8 & 43.8 & 43.6 & 0.4 & 7.65 & 7.50 & 7.50 & 7.55 & 0.09 \\
\hline & I944 & & & & & & & & & & \\
\hline & I946 & 43.9 & 44.3 & 44.3 & 44.2 & 0.2 & 7.76 & 7.77 & 7.72 & 7.75 & 0.02 \\
\hline & I947 & & & & & & & & & & \\
\hline & I950 & & & & & & & & & & \\
\hline & I952 & 46.8 & 47.3 & 48.4 & 47.5 & 0.9 & 9.14 & 10.08 & 10.17 & 9.80 & 0.57 \\
\hline & I953 & 22.3 & 24.4 & 21.8 & 22.8 & 1.4 & 3.47 & 4.40 & 4.29 & 4.05 & 0.51 \\
\hline & I954 & 23.7 & 23.6 & 23.6 & 23.6 & 0.1 & 4.37 & 4.44 & 4.39 & 4.40 & 0.04 \\
\hline & I956 & & & & & & & & & & \\
\hline & I957 & & & & & & & & & & \\
\hline & I958 & & & & & & & & & & \\
\hline & I963 & 47.6 & 48.5 & 47.1 & 47.7 & 0.7 & 11.29 & 9.34 & 11.08 & 10.57 & 1.07 \\
\hline & I964 & 40.1 & 39.7 & 40.4 & 40.1 & 0.4 & 7.17 & 6.84 & 6.71 & 6.91 & 0.24 \\
\hline & I965 & & & & & & & & & & \\
\hline & I966 & 9.7 & 8.8 & 8.6 & 9.0 & 0.6 & 8.60 & 9.50 & 8.90 & 9.00 & 0.46 \\
\hline & I968 & 49.2 & 49.1 & 45.7 & 48.0 & 2.0 & 9.70 & 9.60 & 9.40 & 9.57 & 0.15 \\
\hline & I969 & & & & & & & & & & \\
\hline & I970 & & & & & & & & & & \\
\hline & I976 & & & & & & & & & & \\
\hline & I979 & & & & & & & & & & \\
\hline & I982 & 42.2 & 44.1 & 42.7 & 43.0 & 1.0 & 7.60 & 8.20 & 7.30 & 7.70 & 0.46 \\
\hline & I984 & & & & & & & & & & \\
\hline & I985 & 43.0 & 42.9 & 43.2 & 43.1 & 0.1 & 7.57 & 7.58 & 7.41 & 7.52 & 0.10 \\
\hline \multirow{5}{*}{ 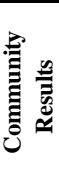 } & & \multicolumn{3}{|c|}{ Consensus Mean } & \multicolumn{2}{|l|}{42.8} & \multicolumn{2}{|c|}{ Consensus Mean } & & 7.59 & \\
\hline & & \multicolumn{3}{|c|}{ Consensus Standard Deviation } & \multicolumn{2}{|l|}{6.4} & \multicolumn{3}{|c|}{ Consensus Standard Deviation } & 1.74 & \\
\hline & & \multicolumn{3}{|c|}{ Maximum } & \multicolumn{2}{|l|}{48.0} & Maximur & & & 10.57 & \\
\hline & & Minimum & & & 9.0 & & Minimun & & & 4.05 & \\
\hline & & $\mathrm{N}$ & & & 12 & & $\mathrm{~N}$ & & & 12 & \\
\hline
\end{tabular}


Table 16. Data summary table for epicatechin gallate in green tea.

\begin{tabular}{|c|c|c|c|c|c|c|c|c|c|c|c|}
\hline & \multirow[b]{3}{*}{ Lab } & \multicolumn{10}{|c|}{ Epicatechin gallate } \\
\hline & & \multicolumn{5}{|c|}{ SRM 3255 Green Tea Extract (mg/g) } & \multicolumn{5}{|c|}{ SRM 3254 Green Tea (mg/g) } \\
\hline & & $\mathbf{A}$ & B & C & Avg & SD & A & B & C & Avg & SD \\
\hline \multirow{50}{*}{ 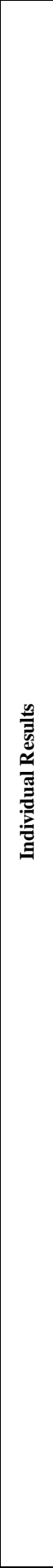 } & NIST & & & & 97.2 & 7.6 & & & & 12.0 & 1.1 \\
\hline & I901 & & & & & & & & & & \\
\hline & I902 & 94.5 & 94.4 & 95.3 & 94.7 & 0.5 & 12.2 & 12.6 & 12.3 & 12.4 & 0.2 \\
\hline & I903 & 99.8 & 99.9 & 101.0 & 100.2 & 0.7 & 15.6 & 15.5 & 15.7 & 15.6 & 0.1 \\
\hline & I904 & & & & & & & & & & \\
\hline & I905 & 98.9 & 99.5 & 98.5 & 99.0 & 0.5 & 14.1 & 14.2 & 14.2 & 14.2 & 0.1 \\
\hline & I906 & & & & & & & & & & \\
\hline & I907 & & & & & & & & & & \\
\hline & I909 & 111.3 & 107.0 & 116.1 & 111.4 & 4.5 & 12.3 & 12.8 & 12.6 & 12.6 & 0.2 \\
\hline & I911 & & & & & & & & & & \\
\hline & I912 & 93.6 & 93.9 & 91.0 & 92.8 & 1.6 & 6.8 & 6.9 & 6.0 & 6.6 & 0.5 \\
\hline & I913 & 20.4 & 16.3 & 22.2 & 19.6 & 3.0 & 1.3 & 1.2 & 1.3 & 1.3 & 0.1 \\
\hline & I914 & & & & & & & & & & \\
\hline & I916 & & & & & & & & & & \\
\hline & I918 & 91.6 & 88.8 & 88.3 & 89.6 & 1.8 & 13.9 & 11.8 & 11.6 & 12.4 & 1.3 \\
\hline & I921 & & & & & & & & & & \\
\hline & I922 & & & & & & & & & & \\
\hline & I923 & 85.8 & 85.0 & 84.8 & 85.2 & 0.5 & 10.4 & 10.7 & 9.6 & 10.2 & 0.6 \\
\hline & I926 & 81.4 & 82.3 & 82.9 & 82.2 & 0.8 & & & & & \\
\hline & I927 & 97.5 & 104.0 & 105.9 & 102.5 & 4.4 & 6.9 & 10.1 & 10.9 & 9.3 & 2.1 \\
\hline & I928 & & & & & & & & & & \\
\hline & I930 & & & & & & & & & & \\
\hline & I933 & 108.5 & 106.2 & 107.0 & 107.2 & 1.1 & 11.0 & 11.0 & 11.0 & 11.0 & 0.0 \\
\hline & I934 & 95.9 & 97.1 & 96.7 & 96.6 & 0.6 & 12.8 & 13.3 & 13.5 & 13.2 & 0.4 \\
\hline & I938 & & & & & & & & & & \\
\hline & I939 & 128.4 & 128.6 & 126.6 & 127.9 & 1.1 & 10.5 & 10.8 & 10.7 & 10.6 & 0.1 \\
\hline & I940 & & & & & & & & & & \\
\hline & I943 & 90.6 & 94.1 & 93.8 & 92.8 & 1.9 & 12.8 & 12.3 & 12.2 & 12.4 & 0.3 \\
\hline & I944 & & & & & & & & & & \\
\hline & I946 & 100.8 & 101.6 & 101.6 & 101.3 & 0.5 & 10.4 & 10.5 & 10.5 & 10.4 & 0.1 \\
\hline & I947 & & & & & & & & & & \\
\hline & I950 & & & & & & & & & & \\
\hline & I952 & 101.1 & 102.3 & 105.4 & 102.9 & 2.2 & 12.5 & 13.7 & 15.8 & 14.0 & 1.6 \\
\hline & I953 & 88.5 & 87.6 & 88.3 & 88.1 & 0.5 & 11.5 & 12.2 & 11.9 & 11.9 & 0.3 \\
\hline & I954 & 3.3 & 3.3 & 3.3 & 3.3 & 0.0 & 0.5 & 0.5 & 0.5 & 0.5 & 0.0 \\
\hline & I956 & & & & & & & & & & \\
\hline & I957 & & & & & & & & & & \\
\hline & I958 & & & & & & & & & & \\
\hline & I963 & 81.6 & 81.7 & 81.0 & 81.4 & 0.4 & 9.9 & 9.8 & 9.7 & 9.8 & 0.1 \\
\hline & I964 & 100.0 & 99.1 & 101.0 & 100.0 & 1.0 & 12.4 & 11.9 & 11.7 & 12.0 & 0.4 \\
\hline & I965 & & & & & & & & & & \\
\hline & I966 & 21.7 & 19.8 & 18.9 & 20.1 & 1.4 & 16.0 & 15.9 & 15.7 & 15.9 & 0.2 \\
\hline & I968 & 104.1 & 101.7 & 100.6 & 102.1 & 1.8 & 14.9 & 15.2 & 15.0 & 15.0 & 0.2 \\
\hline & I969 & & & & & & & & & & \\
\hline & I970 & & & & & & & & & & \\
\hline & I976 & & & & & & & & & & \\
\hline & I979 & & & & & & & & & & \\
\hline & I982 & 85.0 & 88.3 & 88.7 & 87.3 & 2.0 & 14.2 & 14.2 & 13.4 & 13.9 & 0.5 \\
\hline & I984 & & & & & & & & & & \\
\hline & I985 & 121.4 & 121.4 & 122.0 & 121.6 & 0.4 & 11.0 & 10.5 & 10.5 & 10.7 & 0.3 \\
\hline \multirow{5}{*}{ 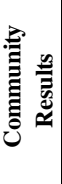 } & & \multicolumn{3}{|c|}{ Consensus Mean } & \multicolumn{2}{|l|}{94.6} & \multicolumn{2}{|c|}{ Consensus Mean } & & 11.8 & \\
\hline & & \multicolumn{3}{|c|}{ Consensus Standard Deviation } & \multicolumn{2}{|l|}{13.8} & \multicolumn{3}{|c|}{ Consensus Standard Deviation } & 2.8 & \\
\hline & & \multicolumn{3}{|c|}{ Maximum } & \multicolumn{2}{|l|}{127.9} & Maximun & & & 15.9 & \\
\hline & & Minimum & & & 3.3 & & Minimum & & & 0.5 & \\
\hline & & $\mathrm{N}$ & & & 12 & & $\mathrm{~N}$ & & & 12 & \\
\hline
\end{tabular}


Table 17. Data summary table for epigallocatechin in green tea.

\begin{tabular}{|c|c|c|c|c|c|c|c|c|c|c|c|}
\hline & \multirow[b]{3}{*}{ Lab } & \multicolumn{10}{|c|}{ Epigallocatechin } \\
\hline & & \multicolumn{5}{|c|}{ SRM 3255 Green Tea Extract (mg/g) } & \multicolumn{5}{|c|}{ SRM 3254 Green Tea (mg/g) } \\
\hline & & $\mathbf{A}$ & B & $\mathbf{C}$ & Avg & SD & A & B & C & Avg & SD \\
\hline \multirow{50}{*}{ 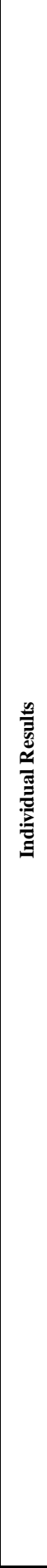 } & NIST & & & & 79.2 & 6.3 & & & & 23.9 & 4.3 \\
\hline & I901 & & & & & & & & & & \\
\hline & I902 & 88.5 & 87.8 & 89.1 & 88.5 & 0.7 & 19.8 & 20.0 & 20.3 & 20.0 & 0.3 \\
\hline & I903 & 77.3 & 76.6 & 77.7 & 77.2 & 0.6 & 27.9 & 26.5 & 27.3 & 27.2 & 0.7 \\
\hline & I904 & & & & & & & & & & \\
\hline & I905 & 88.7 & 88.7 & 88.3 & 88.6 & 0.2 & 29.0 & 29.5 & 29.2 & 29.2 & 0.3 \\
\hline & I906 & & & & & & & & & & \\
\hline & I907 & & & & & & & & & & \\
\hline & I909 & 78.0 & 79.9 & 80.5 & 79.5 & 1.3 & 18.2 & 20.1 & 19.8 & 19.4 & 1.0 \\
\hline & I911 & & & & & & & & & & \\
\hline & I912 & 30.1 & 30.1 & 29.3 & 29.8 & 0.5 & 4.7 & 4.6 & 4.6 & 4.6 & 0.1 \\
\hline & I913 & 1.3 & 1.0 & 1.4 & 1.3 & 0.2 & 0.2 & 0.2 & 0.2 & 0.2 & 0.0 \\
\hline & I914 & & & & & & & & & & \\
\hline & I916 & & & & & & & & & & \\
\hline & I918 & 68.2 & 70.0 & 71.3 & 69.8 & 1.6 & 24.6 & 22.6 & 21.3 & 22.8 & 1.7 \\
\hline & I921 & & & & & & & & & & \\
\hline & I922 & & & & & & & & & & \\
\hline & I923 & 72.7 & 72.9 & 70.7 & 72.1 & 1.3 & 18.0 & 19.1 & 17.0 & 18.0 & 1.0 \\
\hline & I926 & 64.9 & 64.7 & 66.0 & 65.2 & 0.7 & & & & & \\
\hline & I927 & 92.4 & 94.6 & 93.2 & 93.4 & 1.1 & 19.9 & 28.9 & 27.9 & 25.6 & 4.9 \\
\hline & I928 & & & & & & & & & & \\
\hline & I930 & & & & & & & & & & \\
\hline & I933 & 75.5 & 75.3 & 75.8 & 75.5 & 0.3 & 20.2 & 21.3 & 20.8 & 20.8 & 0.6 \\
\hline & I934 & 79.5 & 80.9 & 80.5 & 80.3 & 0.7 & 23.3 & 25.8 & 26.3 & 25.1 & 1.6 \\
\hline & I938 & & & & & & & & & & \\
\hline & I939 & 14.0 & 13.9 & 13.9 & 13.9 & 0.1 & 4.4 & 4.4 & 4.4 & 4.4 & 0.0 \\
\hline & I940 & & & & & & & & & & \\
\hline & I943 & 68.4 & 70.5 & 70.4 & 69.8 & 1.2 & 24.6 & 23.3 & 22.9 & 23.6 & 0.9 \\
\hline & I944 & & & & & & & & & & \\
\hline & I946 & 74.1 & 74.9 & 75.1 & 74.7 & 0.5 & 25.1 & 25.1 & 25.0 & 25.1 & 0.0 \\
\hline & I947 & & & & & & & & & & \\
\hline & I950 & & & & & & & & & & \\
\hline & I952 & 74.6 & 74.9 & 77.2 & 75.6 & 1.4 & 26.4 & 27.6 & 26.6 & 26.9 & 0.7 \\
\hline & I953 & 49.6 & 51.0 & 50.3 & 50.3 & 0.7 & 15.5 & 16.7 & 16.6 & 16.2 & 0.7 \\
\hline & I954 & 15.7 & 15.6 & 15.5 & 15.6 & 0.1 & 4.2 & 4.3 & 4.3 & 4.3 & 0.1 \\
\hline & I956 & & & & & & & & & & \\
\hline & I957 & & & & & & & & & & \\
\hline & I958 & & & & & & & & & & \\
\hline & I963 & 123.1 & 123.5 & 126.4 & 124.3 & 1.8 & 36.0 & 36.2 & 35.4 & 35.9 & 0.4 \\
\hline & I964 & 89.0 & 88.4 & 89.8 & 89.1 & 0.7 & 27.2 & 25.7 & 25.2 & 26.0 & 1.0 \\
\hline & I965 & & & & & & & & & & \\
\hline & I966 & 2.0 & 1.8 & 2.0 & 1.9 & 0.1 & 44.2 & 43.7 & 43.5 & 43.8 & 0.4 \\
\hline & I968 & 94.7 & 86.5 & 83.1 & 88.1 & 6.0 & 38.0 & 37.2 & 36.7 & 37.3 & 0.7 \\
\hline & I969 & & & & & & & & & & \\
\hline & I970 & & & & & & & & & & \\
\hline & I976 & & & & & & & & & & \\
\hline & I979 & & & & & & & & & & \\
\hline & I982 & & & & & & & & & & \\
\hline & I984 & & & & & & & & & & \\
\hline & I985 & 11.7 & 11.8 & 11.8 & 11.8 & 0.1 & 3.8 & 3.8 & 3.7 & 3.8 & 0.0 \\
\hline \multirow{5}{*}{ 象 } & & \multicolumn{3}{|c|}{ Consensus Mean } & \multicolumn{2}{|l|}{63.8} & \multicolumn{2}{|c|}{ Consensus Mean } & & 20.8 & \\
\hline & & \multicolumn{3}{|c|}{ Consensus Standard Deviation } & \multicolumn{2}{|l|}{33.3} & \multicolumn{3}{|c|}{ Consensus Standard Deviation } & 12.5 & \\
\hline & & \multicolumn{3}{|c|}{ Maximum } & \multicolumn{2}{|l|}{124.3} & Maximu & & & 43.8 & \\
\hline & & Minimum & & & 1.9 & & Minimun & & & 3.8 & \\
\hline & & $\mathrm{N}$ & & & 11 & & $\mathrm{~N}$ & & & 11 & \\
\hline
\end{tabular}


Table 18. Data summary table for epigallocatechin gallate in green tea.

\begin{tabular}{|c|c|c|c|c|c|c|c|c|c|c|c|}
\hline & \multirow[b]{3}{*}{ Lab } & \multicolumn{10}{|c|}{ Epigallocatechin gallate } \\
\hline & & \multicolumn{5}{|c|}{ SRM 3255 Green Tea Extract (mg/g) } & \multicolumn{5}{|c|}{ SRM 3254 Green Tea (mg/g) } \\
\hline & & A & B & $\mathbf{C}$ & Avg & SD & A & B & C & Avg & SD \\
\hline \multirow{50}{*}{ 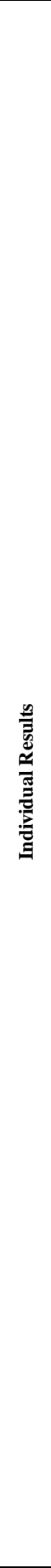 } & NIST & & & & 409 & 18 & & & & 49.3 & 2.1 \\
\hline & I901 & & & & & & & & & & \\
\hline & I902 & 409 & 407 & 408 & 408 & 1 & 43.3 & 43.6 & 44.5 & 43.8 & 0.6 \\
\hline & I903 & 420 & 418 & 422 & 420 & 2 & 58.9 & 58.1 & 59.2 & 58.7 & 0.6 \\
\hline & I904 & & & & & & & & & & \\
\hline & I905 & 458 & 462 & 457 & 459 & 3 & 59.6 & 60.1 & 60.5 & 60.1 & 0.5 \\
\hline & I906 & & & & & & & & & & \\
\hline & I907 & & & & & & & & & & \\
\hline & I909 & 358 & 375 & 394 & 376 & 18 & 65.2 & 63.5 & 64.4 & 64.4 & 0.8 \\
\hline & I911 & & & & & & & & & & \\
\hline & I912 & 415 & 420 & 409 & 415 & 5 & 31.7 & 36.5 & 28.1 & 32.1 & 4.2 \\
\hline & I913 & 56 & 45 & 60 & 54 & 8 & 2.7 & 2.6 & 2.8 & 2.7 & 0.1 \\
\hline & I914 & & & & & & & & & & \\
\hline & I916 & & & & & & & & & & \\
\hline & I918 & 424 & 412 & 410 & 416 & 8 & 51.9 & 49.1 & 47.5 & 49.5 & 2.2 \\
\hline & I921 & & & & & & & & & & \\
\hline & I922 & & & & & & & & & & \\
\hline & I923 & 373 & 365 & 365 & 368 & 5 & 35.9 & 37.7 & 33.2 & 35.6 & 2.3 \\
\hline & I926 & 349 & 351 & 354 & 351 & 2 & & & & & \\
\hline & I927 & 402 & 411 & 408 & 407 & 4 & 34.2 & 50.1 & 50.1 & 44.8 & 9.2 \\
\hline & I928 & 428 & 426 & 432 & 429 & 3 & 57.3 & 54.8 & 56.3 & 56.1 & 1.3 \\
\hline & I930 & & & & & & & & & & \\
\hline & I933 & 404 & 402 & 400 & 402 & 2 & 37.1 & 38.3 & 37.8 & 37.7 & 0.6 \\
\hline & I934 & 409 & 412 & 411 & 411 & 2 & 52.5 & 52.6 & 53.6 & 52.9 & 0.6 \\
\hline & I938 & & & & & & & & & & \\
\hline & I939 & 462 & 464 & 460 & 462 & 2 & 39.5 & 40.6 & 40.2 & 40.1 & 0.6 \\
\hline & I940 & 437 & 429 & 434 & 434 & 4 & 45.5 & 45.1 & 46.5 & 45.7 & 0.7 \\
\hline & I943 & 395 & 400 & 399 & 398 & 2 & 49.0 & 47.5 & 46.8 & 47.8 & 1.1 \\
\hline & I944 & & & & & & & & & & \\
\hline & I946 & 402 & 407 & 407 & 405 & 3 & 41.9 & 42.1 & 42.2 & 42.1 & 0.2 \\
\hline & I947 & & & & & & & & & & \\
\hline & I950 & & & & & & & & & & \\
\hline & I952 & 404 & 409 & 421 & 411 & 9 & 48.7 & 53.1 & 58.3 & 53.4 & 4.8 \\
\hline & I953 & 356 & 367 & 358 & 360 & 6 & 38.0 & 42.2 & 42.1 & 40.8 & 2.4 \\
\hline & I954 & 438 & 437 & 434 & 436 & 2 & 59.5 & 60.6 & 59.5 & 59.9 & 0.6 \\
\hline & I956 & & & & & & & & & & \\
\hline & I957 & & & & & & & & & & \\
\hline & I958 & & & & & & & & & & \\
\hline & I963 & 408 & 403 & 399 & 403 & 5 & 45.6 & 45.5 & 45.5 & 45.5 & 0.1 \\
\hline & I964 & 436 & 433 & 441 & 437 & 4 & 49.2 & 46.8 & 46.1 & 47.4 & 1.6 \\
\hline & I965 & & & & & & & & & & \\
\hline & I966 & 89 & 82 & 78 & 83 & 6 & 54.6 & 53.4 & 51.7 & 53.2 & 1.5 \\
\hline & I968 & 406 & 406 & 399 & 404 & 4 & 60.2 & 59.6 & 58.5 & 59.4 & 0.9 \\
\hline & I969 & & & & & & & & & & \\
\hline & I970 & & & & & & & & & & \\
\hline & I976 & & & & & & & & & & \\
\hline & I979 & & & & & & & & & & \\
\hline & I982 & 449 & 465 & 463 & 459 & 9 & 66.3 & 67.5 & 62.5 & 65.4 & 2.6 \\
\hline & I984 & & & & & & & & & & \\
\hline & I985 & 463 & 464 & 466 & 464 & 1 & 40.3 & 39.3 & 38.8 & 39.4 & 0.8 \\
\hline \multirow{5}{*}{ 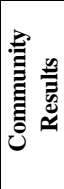 } & & \multicolumn{3}{|c|}{ Consensus Mean } & \multicolumn{2}{|l|}{409} & \multicolumn{2}{|c|}{ Consensus Mean } & & 48.3 & \\
\hline & & \multicolumn{3}{|c|}{ Consensus Standard Deviation } & \multicolumn{2}{|l|}{39} & \multicolumn{3}{|c|}{ Consensus Standard Deviation } & 11.0 & \\
\hline & & \multicolumn{3}{|c|}{ Maximum } & \multicolumn{2}{|l|}{464} & Maximu & & & 65.4 & \\
\hline & & Minimun & & & 83 & & Minimun & & & 39.4 & \\
\hline & & $\mathrm{N}$ & & & 13 & & $\mathrm{~N}$ & & & 13 & \\
\hline
\end{tabular}


Table 19. Data summary table for gallocatechin in green tea.

\begin{tabular}{|c|c|c|c|c|c|c|c|c|c|c|c|}
\hline & \multirow[b]{3}{*}{ Lab } & \multicolumn{10}{|c|}{ Gallocatechin } \\
\hline & & \multicolumn{5}{|c|}{ SRM 3255 Green Tea Extract (mg/g) } & \multicolumn{5}{|c|}{ SRM 3254 Green Tea (mg/g) } \\
\hline & & $\mathbf{A}$ & B & C & Avg & SD & A & B & C & Avg & SD \\
\hline \multirow{50}{*}{ 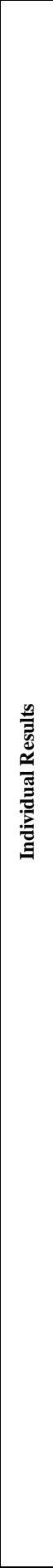 } & NIST & & & & 21.3 & 1.6 & & & & 2.28 & 1.04 \\
\hline & I901 & & & & & & & & & & \\
\hline & I902 & & & & & & & & & & \\
\hline & I903 & 12.9 & 12.6 & 12.5 & 12.7 & 0.2 & 6.57 & 6.84 & 7.15 & 6.85 & 0.29 \\
\hline & I904 & & & & & & & & & & \\
\hline & I905 & 24.3 & 24.2 & 24.3 & 24.3 & 0.1 & 4.20 & 4.10 & 4.20 & 4.17 & 0.06 \\
\hline & I906 & & & & & & & & & & \\
\hline & I907 & & & & & & & & & & \\
\hline & I909 & 14.6 & 17.1 & 18.8 & 16.8 & 2.1 & 1.13 & 1.12 & 1.07 & 1.10 & 0.03 \\
\hline & I911 & & & & & & & & & & \\
\hline & I912 & 99.9 & 100.1 & 100.4 & 100.1 & 0.3 & 31.15 & 29.03 & 29.11 & 29.76 & 1.20 \\
\hline & I913 & 0.1 & & 0.1 & 0.1 & 0.0 & 0.02 & 0.02 & & 0.02 & 0.00 \\
\hline & I914 & & & & & & & & & & \\
\hline & I916 & & & & & & & & & & \\
\hline & I918 & 5.9 & 5.7 & 5.8 & 5.8 & 0.1 & 0.30 & 0.26 & 0.29 & 0.28 & 0.02 \\
\hline & I921 & & & & & & & & & & \\
\hline & I922 & & & & & & & & & & \\
\hline & I923 & 18.1 & 19.7 & 19.3 & 19.0 & 0.8 & 1.78 & 2.17 & 1.82 & 1.92 & 0.21 \\
\hline & I926 & & & & & & & & & & \\
\hline & I927 & 28.2 & 29.3 & 29.1 & 28.9 & 0.6 & 3.14 & 4.00 & 4.52 & 3.89 & 0.70 \\
\hline & I928 & & & & & & & & & & \\
\hline & I930 & & & & & & & & & & \\
\hline & I933 & 113.7 & 113.2 & 115.6 & 114.2 & 1.2 & 17.48 & 17.07 & 17.61 & 17.39 & 0.28 \\
\hline & I934 & 22.6 & 23.3 & 22.5 & 22.8 & 0.4 & 2.41 & 2.43 & 2.61 & 2.48 & 0.11 \\
\hline & I938 & & & & & & & & & & \\
\hline & I939 & & & & & & & & & & \\
\hline & I940 & & & & & & & & & & \\
\hline & I943 & & & & & & & & & & \\
\hline & I944 & & & & & & & & & & \\
\hline & I946 & 26.1 & 26.4 & 26.4 & 26.3 & 0.2 & 4.08 & 4.09 & 4.07 & 4.08 & 0.01 \\
\hline & I947 & & & & & & & & & & \\
\hline & I950 & & & & & & & & & & \\
\hline & I952 & 22.8 & 22.9 & 23.6 & 23.1 & 0.5 & 3.72 & 5.40 & 3.66 & 4.26 & 0.99 \\
\hline & I953 & 15.4 & 24.5 & 22.2 & 20.7 & 4.8 & 6.13 & 5.86 & 6.34 & 6.11 & 0.24 \\
\hline & I954 & 131.0 & 131.0 & 129.0 & 130.3 & 1.2 & 19.40 & 19.70 & 19.30 & 19.47 & 0.21 \\
\hline & I956 & & & & & & & & & & \\
\hline & I957 & & & & & & & & & & \\
\hline & I958 & & & & & & & & & & \\
\hline & I963 & 28.7 & 28.9 & 28.6 & 28.7 & 0.2 & 4.52 & 4.51 & 4.46 & 4.50 & 0.03 \\
\hline & I964 & & & & & & & & & & \\
\hline & I965 & & & & & & & & & & \\
\hline & I966 & & & & & & & & & & \\
\hline & I968 & 47.9 & 55.5 & 45.9 & 49.8 & 5.1 & 27.78 & 27.36 & 29.06 & 28.07 & 0.89 \\
\hline & I969 & & & & & & & & & & \\
\hline & I970 & & & & & & & & & & \\
\hline & I976 & & & & & & & & & & \\
\hline & I979 & & & & & & & & & & \\
\hline & I982 & 22.3 & 23.4 & 22.9 & 22.9 & 0.6 & 3.30 & 2.60 & 2.21 & 2.70 & 0.55 \\
\hline & I984 & & & & & & & & & & \\
\hline & I985 & & & & & & & & & & \\
\hline \multirow{5}{*}{ 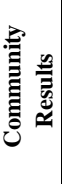 } & & \multicolumn{3}{|c|}{ Consensus Mean } & \multicolumn{2}{|l|}{26.6} & \multicolumn{2}{|c|}{ Consensus Mean } & & 5.42 & \\
\hline & & \multicolumn{3}{|c|}{ Consensus Standard Deviation } & \multicolumn{2}{|l|}{16.9} & \multicolumn{3}{|c|}{ Consensus Standard Deviation } & 4.99 & \\
\hline & & Maximun & & & 130.3 & & Maximun & & & 28.07 & \\
\hline & & Minimum & & & 20.7 & & Minimum & & & 2.70 & \\
\hline & & $\mathrm{N}$ & & & 7 & & $\mathrm{~N}$ & & & 7 & \\
\hline
\end{tabular}


Table 20. Data summary table for gallocatechin gallate in green tea.

\begin{tabular}{|c|c|c|c|c|c|c|c|c|c|c|c|}
\hline & \multirow[b]{3}{*}{ Lab } & \multicolumn{10}{|c|}{ Gallocatechin gallate } \\
\hline & & \multicolumn{5}{|c|}{ SRM 3255 Green Tea Extract (mg/g) } & \multicolumn{5}{|c|}{ SRM 3254 Green Tea (mg/g) } \\
\hline & & A & B & C & Avg & SD & A & B & C & Avg & SD \\
\hline \multirow{50}{*}{ 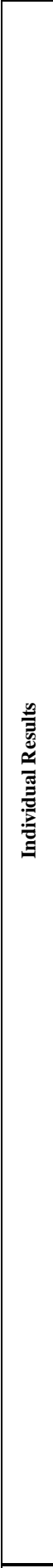 } & NIST & & & & 37.8 & 1.9 & & & & 0.939 & 0.199 \\
\hline & I901 & & & & & & & & & & \\
\hline & I902 & 44.8 & 45.2 & 46.2 & 45.4 & 0.7 & 1.910 & 1.840 & 2.090 & 1.947 & 0.129 \\
\hline & I903 & 42.8 & 42.8 & 42.9 & 42.8 & 0.1 & 1.260 & 1.190 & 1.160 & 1.203 & 0.051 \\
\hline & I904 & & & & & & & & & & \\
\hline & I905 & 1.4 & 1.4 & 1.5 & 1.4 & 0.1 & 0.100 & 0.100 & 0.100 & 0.100 & 0.000 \\
\hline & I906 & & & & & & & & & & \\
\hline & I907 & & & & & & & & & & \\
\hline & I909 & 37.7 & 37.2 & 49.1 & 41.3 & 6.7 & 1.279 & 1.303 & 1.282 & 1.288 & 0.013 \\
\hline & I911 & & & & & & & & & & \\
\hline & I912 & 36.0 & 36.5 & 35.2 & 35.9 & 0.7 & & & & & \\
\hline & I913 & 7.0 & 5.5 & 7.5 & 6.7 & 1.0 & 0.060 & 0.060 & 0.060 & 0.060 & 0.000 \\
\hline & I914 & & & & & & & & & & \\
\hline & I916 & & & & & & & & & & \\
\hline & I918 & 43.0 & 42.2 & 42.1 & 42.4 & 0.5 & 1.129 & 1.163 & 1.281 & 1.191 & 0.080 \\
\hline & I921 & & & & & & & & & & \\
\hline & I922 & & & & & & & & & & \\
\hline & I923 & 41.6 & 41.2 & 41.1 & 41.3 & 0.3 & 1.120 & 1.140 & 1.030 & 1.097 & 0.059 \\
\hline & I926 & 42.2 & 41.9 & 42.5 & 42.2 & 0.3 & & & & & \\
\hline & I927 & 101.5 & 106.1 & 105.2 & 104.3 & 2.5 & 2.700 & 2.473 & 4.277 & 3.150 & 0.983 \\
\hline & I928 & & & & & & & & & & \\
\hline & I930 & & & & & & & & & & \\
\hline & I933 & 61.2 & 52.3 & 52.6 & 55.4 & 5.1 & 1.310 & 1.380 & 1.360 & 1.350 & 0.036 \\
\hline & I934 & 53.4 & 54.2 & 54.1 & 53.9 & 0.4 & 1.205 & 1.147 & 1.266 & 1.206 & 0.060 \\
\hline & I938 & & & & & & & & & & \\
\hline & I939 & 39.2 & 38.6 & 38.4 & 38.7 & 0.4 & 0.877 & 0.990 & 1.044 & 0.971 & 0.085 \\
\hline & I940 & & & & & & & & & & \\
\hline & I943 & & & & & & & & & & \\
\hline & I944 & & & & & & & & & & \\
\hline & I946 & 55.3 & 55.3 & 55.6 & 55.4 & 0.2 & 1.776 & 1.795 & 1.785 & 1.786 & 0.010 \\
\hline & I947 & & & & & & & & & & \\
\hline & I950 & & & & & & & & & & \\
\hline & I952 & 42.4 & 42.8 & 43.9 & 43.0 & 0.8 & 2.060 & 2.020 & 2.510 & 2.197 & 0.272 \\
\hline & I953 & 15.8 & 16.9 & 15.4 & 16.0 & 0.8 & 0.414 & 0.041 & 0.381 & 0.279 & 0.206 \\
\hline & I954 & 47.2 & 47.1 & 46.6 & 47.0 & 0.3 & 1.420 & 1.440 & 1.370 & 1.410 & 0.036 \\
\hline & I956 & & & & & & & & & & \\
\hline & I957 & & & & & & & & & & \\
\hline & I958 & & & & & & & & & & \\
\hline & I963 & 57.8 & 55.3 & 55.0 & 56.0 & 1.5 & 2.616 & 2.430 & 2.560 & 2.535 & 0.095 \\
\hline & I964 & 41.9 & 41.7 & 42.6 & 42.1 & 0.5 & 1.040 & 1.000 & 0.950 & 0.997 & 0.045 \\
\hline & I965 & & & & & & & & & & \\
\hline & I966 & & & & & & & & & & \\
\hline & I968 & 52.7 & 53.0 & 49.0 & 51.6 & 2.2 & 4.266 & 4.268 & 3.085 & 3.873 & 0.682 \\
\hline & I969 & & & & & & & & & & \\
\hline & I970 & & & & & & & & & & \\
\hline & I976 & & & & & & & & & & \\
\hline & I979 & & & & & & & & & & \\
\hline & I982 & 37.1 & 37.8 & 38.4 & 37.7 & 0.7 & & 0.800 & & 0.800 & \\
\hline & I984 & & & & & & & & & & \\
\hline & I985 & 35.4 & 35.7 & 35.9 & 35.7 & 0.2 & 1.314 & 1.234 & 1.116 & 1.221 & 0.100 \\
\hline \multirow{5}{*}{ 象 } & & \multicolumn{3}{|c|}{ Consensus Mean } & \multicolumn{2}{|l|}{43.1} & \multicolumn{2}{|c|}{ Consensus Mean } & & 1.342 & \\
\hline & & \multicolumn{3}{|c|}{ Consensus Standard Deviation } & \multicolumn{2}{|l|}{10.6} & \multicolumn{3}{|c|}{ Consensus Standard Deviation } & 0.847 & \\
\hline & & \multicolumn{3}{|c|}{ Maximum } & \multicolumn{2}{|l|}{56.0} & Maximun & & & 3.873 & \\
\hline & & Minimun & & & 16.0 & & Minimum & & & 0.279 & \\
\hline & & $\mathrm{N}$ & & & 10 & & $\mathrm{~N}$ & & & 9 & \\
\hline
\end{tabular}


Table 21. Data summary table for total catechins in green tea.

\begin{tabular}{|c|c|c|c|c|c|c|c|c|c|c|c|}
\hline & \multirow[b]{3}{*}{ Lab } & \multicolumn{10}{|c|}{ Total catechins } \\
\hline & & \multicolumn{5}{|c|}{ SRM 3255 Green Tea Extract (mg/g) } & \multicolumn{5}{|c|}{ SRM 3254 Green Tea (mg/g) } \\
\hline & & $\mathbf{A}$ & B & C & Avg & SD & $\mathbf{A}$ & B & C & Avg & SD \\
\hline \multirow{50}{*}{ 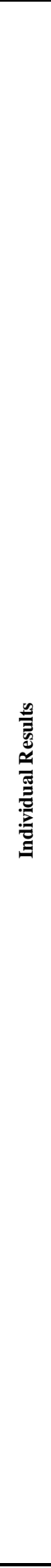 } & NIST & & & & 699 & 22 & & & & 97.9 & 5.2 \\
\hline & I901 & & & & & & & & & & \\
\hline & I902 & 828 & 825 & 829 & 827 & 2 & 155.7 & 156.9 & 157.6 & 156.7 & 1.0 \\
\hline & I903 & 712 & 709 & 715 & 712 & 3 & 120.0 & 118.0 & 120.0 & 119.3 & 1.2 \\
\hline & I904 & & & & & & & & & & \\
\hline & I905 & 734 & 739 & 732 & 735 & 3 & 117.4 & 118.5 & 118.5 & 118.1 & 0.6 \\
\hline & I906 & & & & & & & & & & \\
\hline & I907 & & & & & & & & & & \\
\hline & I909 & 638 & 659 & 705 & 668 & 34 & 106.0 & 106.4 & 106.8 & 106.4 & 0.4 \\
\hline & I911 & & & & & & & & & & \\
\hline & I912 & 733 & 739 & 722 & 731 & 8 & 84.8 & 86.8 & 77.6 & 83.1 & 4.8 \\
\hline & I913 & 88 & 70 & 94 & 84 & 13 & 4.5 & 4.2 & 4.6 & 4.4 & 0.2 \\
\hline & I914 & & & & & & & & & & \\
\hline & I916 & & & & & & & & & & \\
\hline & I918 & 690 & 676 & 674 & 680 & 9 & 101.0 & 93.6 & 90.3 & 95.0 & 5.5 \\
\hline & I921 & & & & & & & & & & \\
\hline & I922 & & & & & & & & & & \\
\hline & I923 & 645 & 640 & 638 & 641 & 4 & 73.9 & 77.8 & 68.8 & 73.5 & 4.5 \\
\hline & I926 & 583 & 586 & 592 & 587 & 4 & & & & & \\
\hline & I927 & 783 & 807 & 802 & 797 & 13 & 73.7 & 104.9 & 107.2 & 95.3 & 18.7 \\
\hline & I928 & & & & & & & & & & \\
\hline & I930 & & & & & & & & & & \\
\hline & I933 & 951 & 936 & 937 & 941 & 8 & 156.0 & 158.7 & 157.8 & 157.5 & 1.4 \\
\hline & I934 & 714 & 722 & 72 & 503 & 373 & 100.5 & 104.0 & 105.6 & 103.3 & 2.6 \\
\hline & I938 & & & & & & & & & & \\
\hline & I939 & 696 & 697 & 691 & 695 & 3 & 65.5 & 66.8 & 66.4 & 66.2 & 0.7 \\
\hline & I940 & & & & & & & & & & \\
\hline & I943 & 608 & 619 & 618 & 615 & 6 & 96.0 & 92.4 & 91.2 & 93.2 & 2.5 \\
\hline & I944 & & & & & & & & & & \\
\hline & I946 & 713 & 720 & 721 & 718 & 4 & 91.9 & 92.3 & 92.2 & 92.1 & 0.2 \\
\hline & I947 & & & & & & & & & & \\
\hline & I950 & & & & & & & & & & \\
\hline & I952 & 701 & 708 & 729 & 713 & 15 & 103.7 & 113.1 & 118.2 & 111.7 & 7.3 \\
\hline & I953 & 663 & 687 & 670 & 673 & 13 & 135.2 & 143.2 & 144.6 & 141.0 & 5.1 \\
\hline & I954 & 664 & 662 & 657 & 661 & 4 & 90.0 & 91.6 & 90.0 & 90.5 & 0.9 \\
\hline & I956 & & & & & & & & & & \\
\hline & I957 & & & & & & & & & & \\
\hline & I958 & & & & & & & & & & \\
\hline & I963 & 753 & 751 & 747 & 750 & 3 & 112.2 & 110.1 & 109.9 & 110.7 & 1.3 \\
\hline & I964 & 715 & 710 & 723 & 716 & 7 & 97.5 & 92.8 & 91.1 & 93.8 & 3.3 \\
\hline & I965 & & & & & & & & & & \\
\hline & I966 & & & & & & & & & & \\
\hline & I968 & 755 & 752 & 723 & 743 & 17 & 154.9 & 153.3 & 151.8 & 153.3 & 1.5 \\
\hline & I969 & & & & & & & & & & \\
\hline & I970 & & & & & & & & & & \\
\hline & I976 & & & & & & & & & & \\
\hline & I979 & & & & & & & & & & \\
\hline & I982 & 649 & 671 & 669 & 663 & 12 & 91.4 & 93.4 & 85.4 & 90.1 & 4.2 \\
\hline & I984 & & & & & & & & & & \\
\hline & I985 & 684 & 685 & 689 & 686 & 2 & 66.9 & 65.4 & 64.6 & 65.7 & 1.2 \\
\hline \multirow{5}{*}{ 象 } & & \multicolumn{3}{|c|}{ Consensus Mean } & \multicolumn{2}{|l|}{694} & \multicolumn{2}{|c|}{ Consensus Mean } & & 102.0 & \\
\hline & & \multicolumn{3}{|c|}{ Consensus Standard Deviation } & \multicolumn{2}{|l|}{73} & \multicolumn{3}{|c|}{ Consensus Standard Deviation } & 29.7 & \\
\hline & & \multicolumn{3}{|c|}{ Maximum } & \multicolumn{2}{|l|}{750} & Maximur & & & 153.3 & \\
\hline & & Minimun & & & 615 & & Minimun & & & 65.7 & \\
\hline & & $\mathrm{N}$ & & & 11 & & $\mathrm{~N}$ & & & 11 & \\
\hline
\end{tabular}




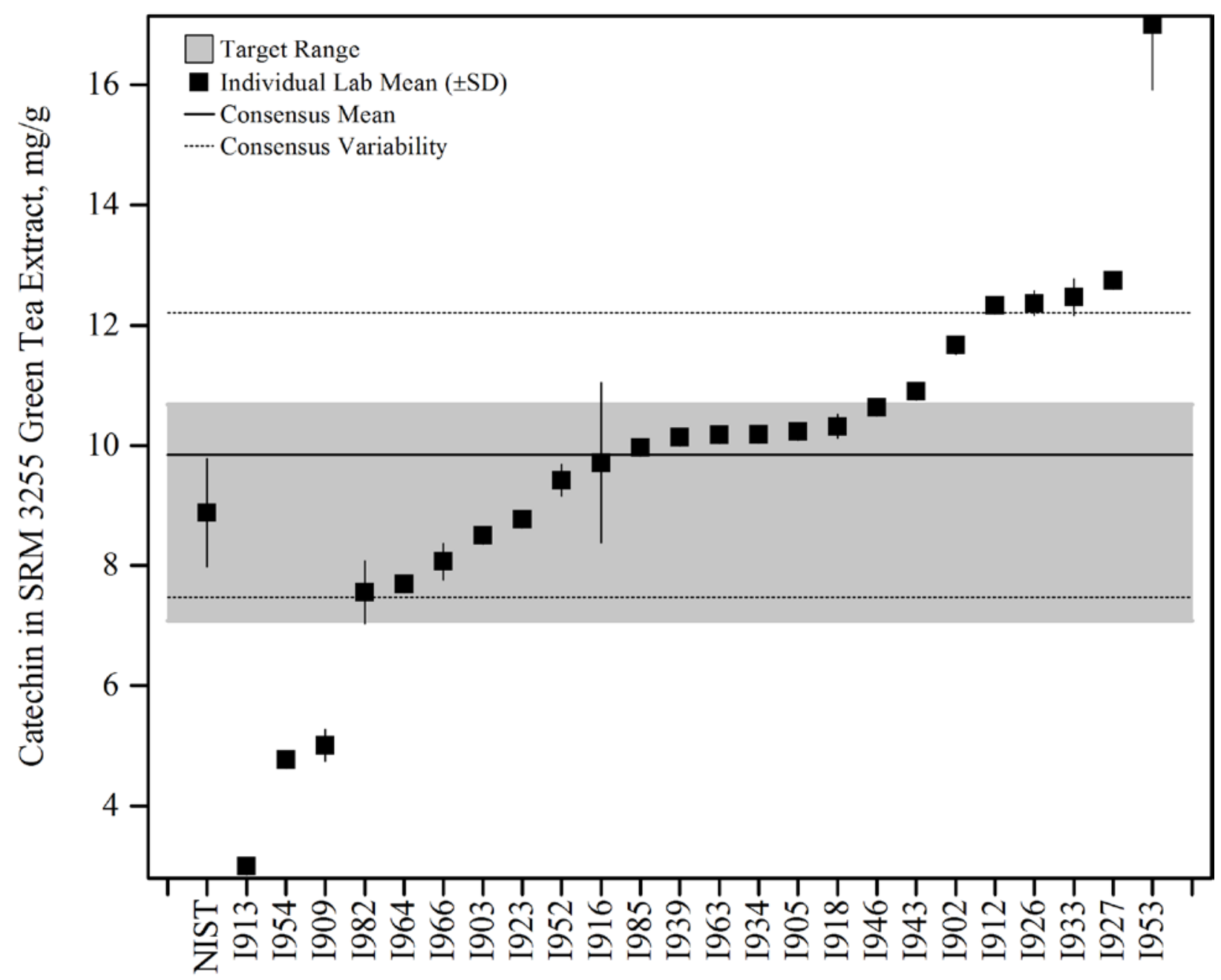

Figure 22. Catechin in SRM 3255 Camellia sinensis (Green Tea) Extract (data summary view). In this view, individual laboratory data are plotted with the individual laboratory standard deviation (error bars). The black solid line represents the consensus mean, and the black dotted lines represent the consensus variability calculated as one standard deviation about the consensus mean. The gray shaded region represents the target zone for "acceptable" performance, which encompasses the NIST certified value bounded by twice its uncertainty $\left(U_{95}\right)$. 


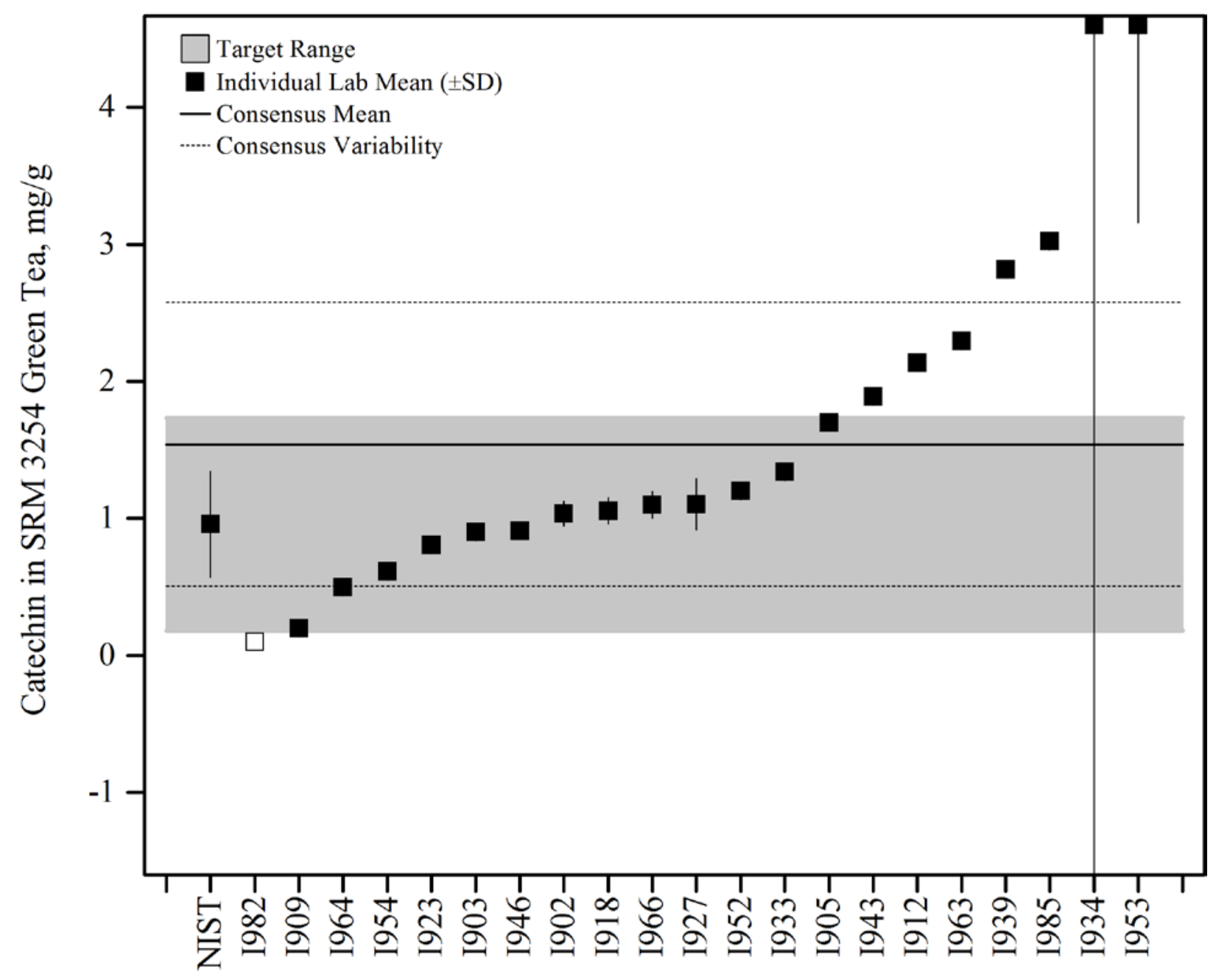

Figure 23. Catechin in SRM 3254 Camellia sinensis (Green Tea) (data summary view). In this view, individual laboratory data are plotted with the individual laboratory standard deviation (error bars). Data points that are unfilled represent laboratories that only reported a single value for that analyte and therefore were not included in the consensus mean. The black solid line represents the consensus mean, and the black dotted lines represent the consensus variability calculated as one standard deviation about the consensus mean. The gray shaded region represents the target zone for "acceptable” performance, which encompasses the NIST certified value bounded by twice its uncertainty $\left(U_{95}\right)$. 


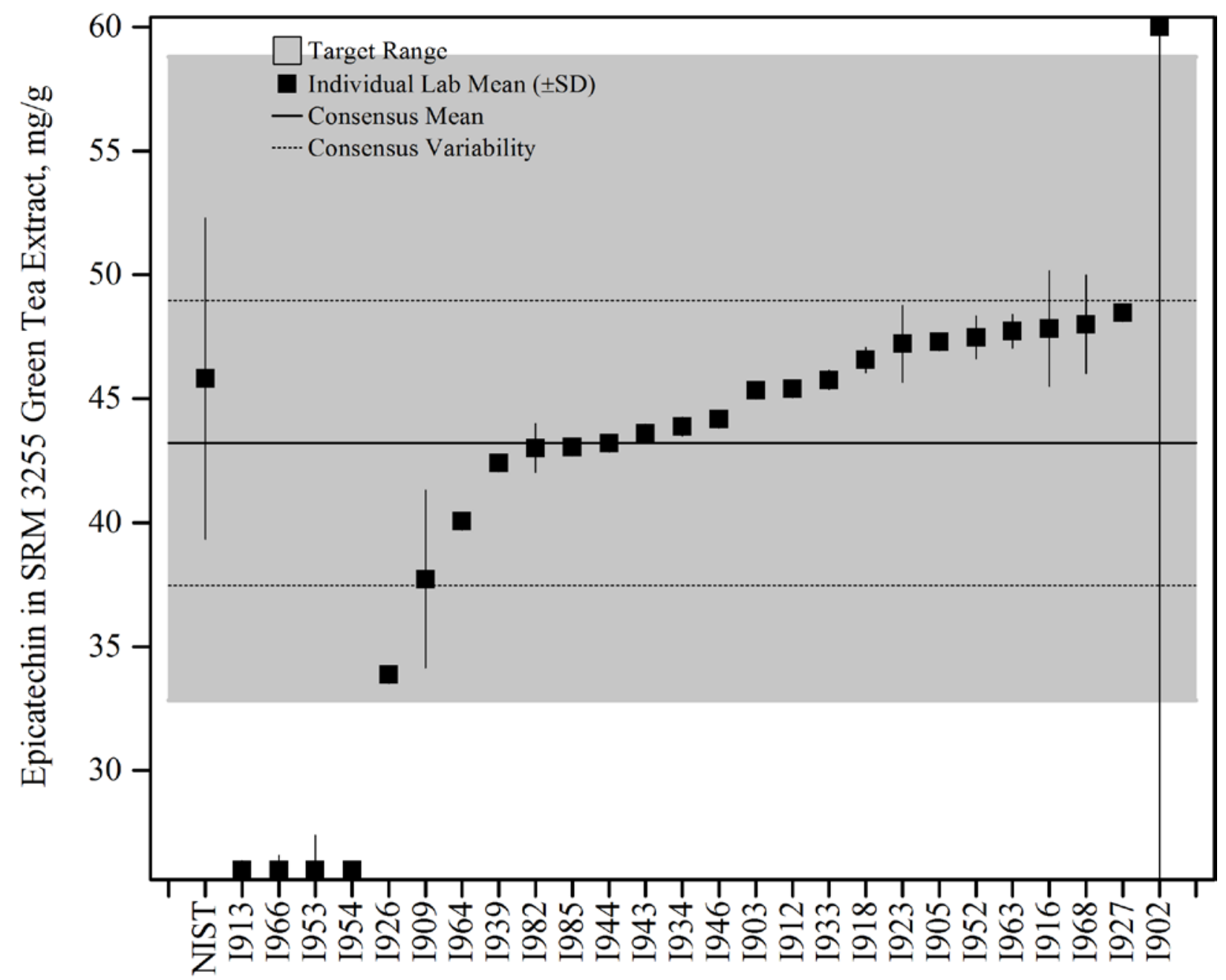

Figure 24. Epicatechin in SRM 3255 Camellia sinensis (Green Tea) Extract (data summary view). In this view, individual laboratory data are plotted with the individual laboratory standard deviation (error bars). The black solid line represents the consensus mean, and the black dotted lines represent the consensus variability calculated as one standard deviation about the consensus mean. The gray shaded region represents the target zone for "acceptable" performance, which encompasses the NIST certified value bounded by twice its uncertainty $\left(U_{95}\right)$. 


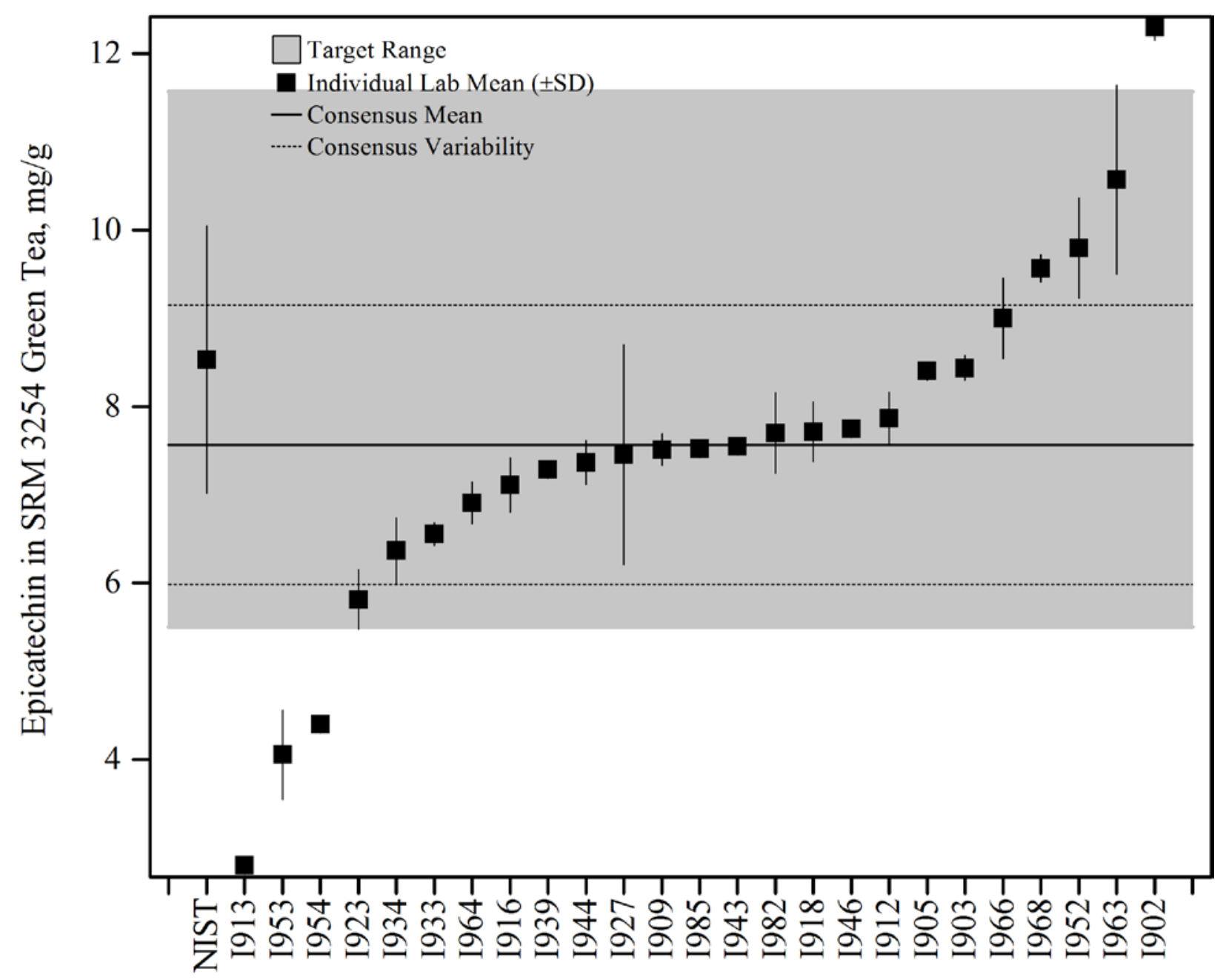

Figure 25. Epicatechin in SRM 3254 Camellia sinensis (Green Tea) (data summary view). In this view, individual laboratory data are plotted with the individual laboratory standard deviation (error bars). The black solid line represents the consensus mean, and the black dotted lines represent the consensus variability calculated as one standard deviation about the consensus mean. The gray shaded region represents the target zone for "acceptable" performance, which encompasses the NIST certified value bounded by twice its uncertainty $\left(U_{95}\right)$. 


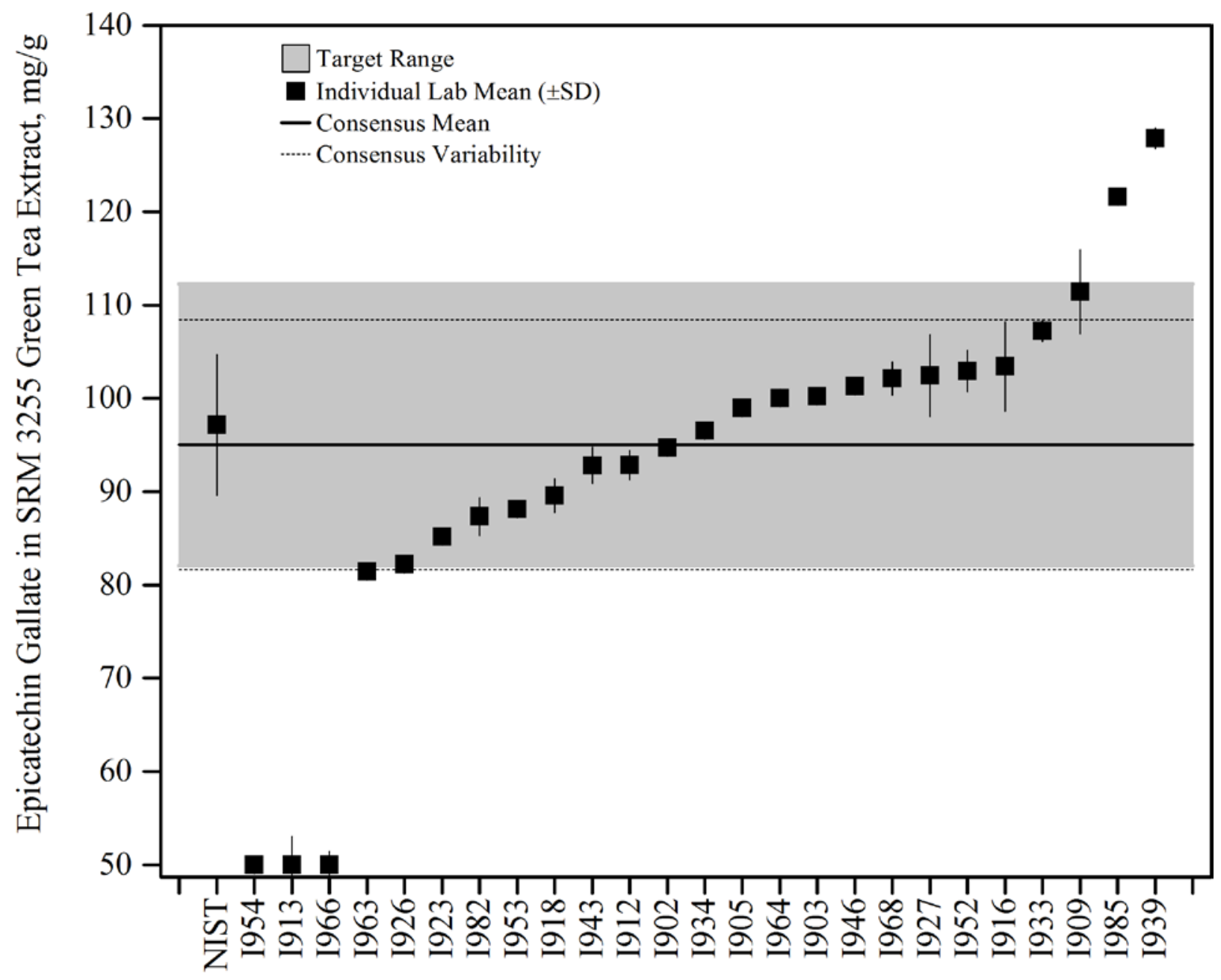

Figure 26. Epicatechin gallate in SRM 3255 Camellia sinensis (Green Tea) Extract (data summary view). In this view, individual laboratory data are plotted with the individual laboratory standard deviation (error bars). The black solid line represents the consensus mean, and the black dotted lines represent the consensus variability calculated as one standard deviation about the consensus mean. The gray shaded region represents the target zone for "acceptable" performance, which encompasses the NIST certified value bounded by twice its uncertainty $\left(U_{95}\right)$. 


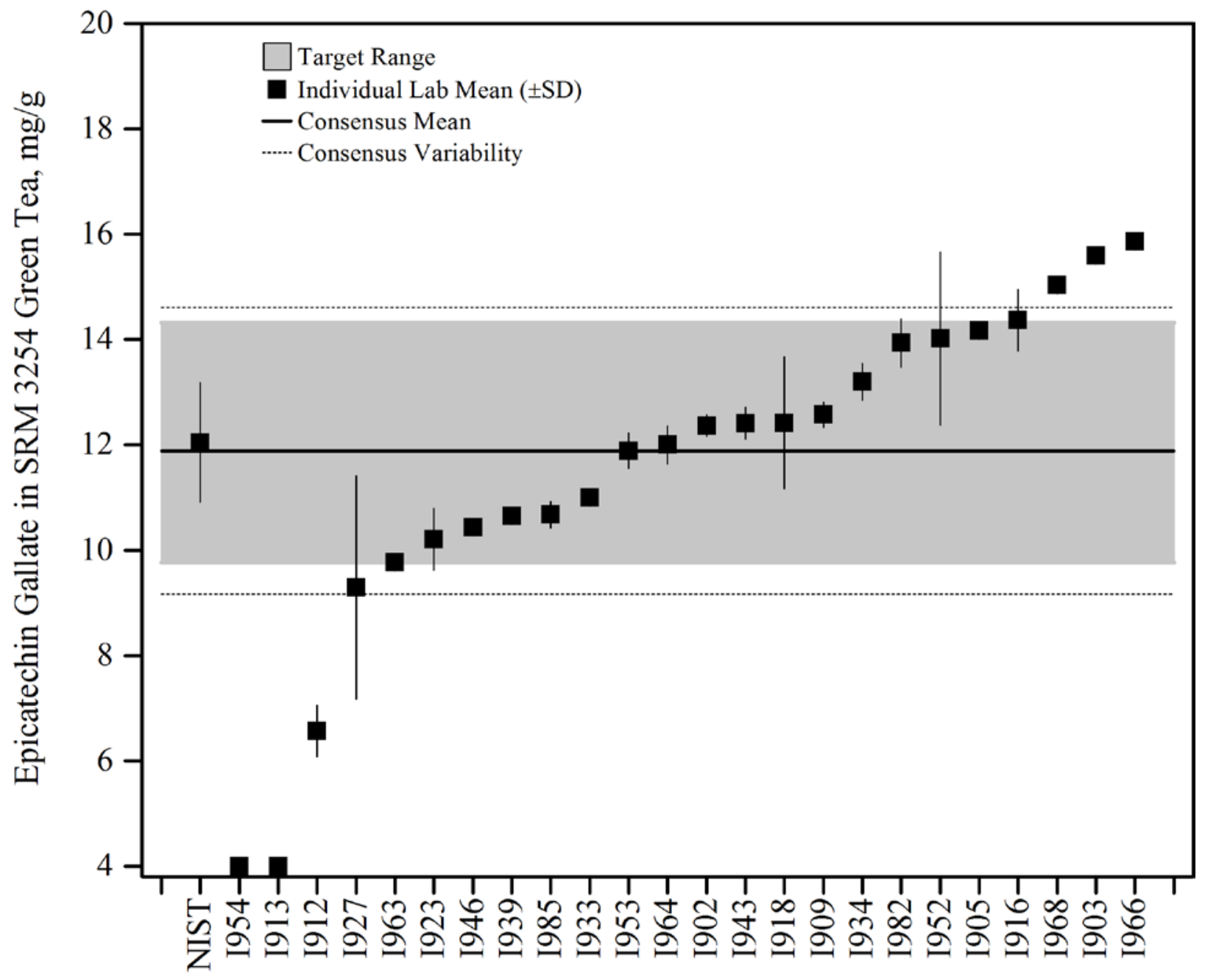

Figure 27. Epicatechin gallate in SRM 3254 Camellia sinensis (Green Tea) (data summary view). In this view, individual laboratory data are plotted with the individual laboratory standard deviation (error bars). The black solid line represents the consensus mean, and the black dotted lines represent the consensus variability calculated as one standard deviation about the consensus mean. The gray shaded region represents the target zone for "acceptable" performance, which encompasses the NIST certified value bounded by twice its uncertainty $\left(U_{95}\right)$. 


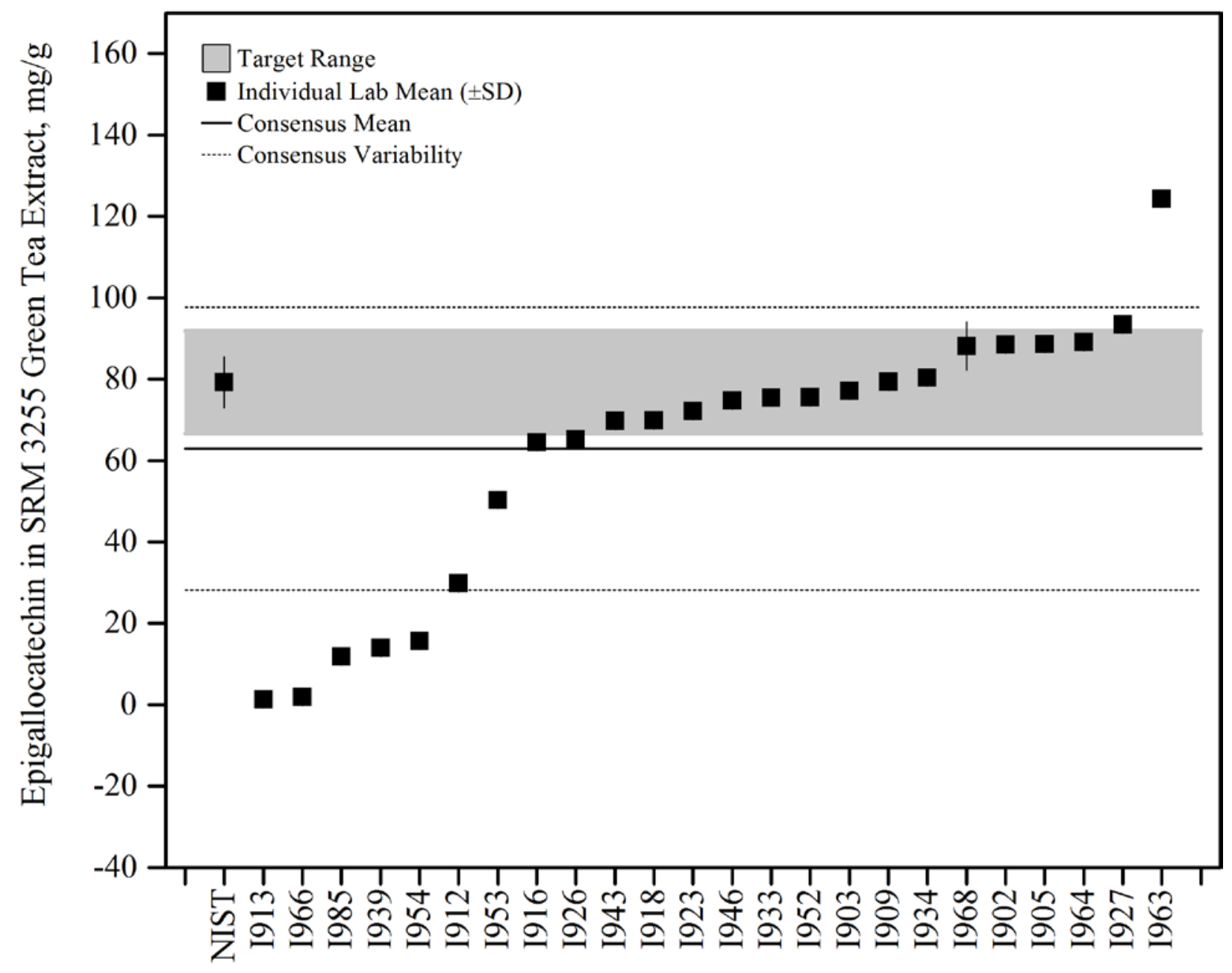

Figure 28. Epigallocatechin in SRM 3255 Camellia sinensis (Green Tea) Extract (data summary view). In this view, individual laboratory data are plotted with the individual laboratory standard deviation (error bars). The black solid line represents the consensus mean, and the black dotted lines represent the consensus variability calculated as one standard deviation about the consensus mean. The gray shaded region represents the target zone for "acceptable” performance, which encompasses the NIST certified value bounded by twice its uncertainty $\left(U_{95}\right)$. 


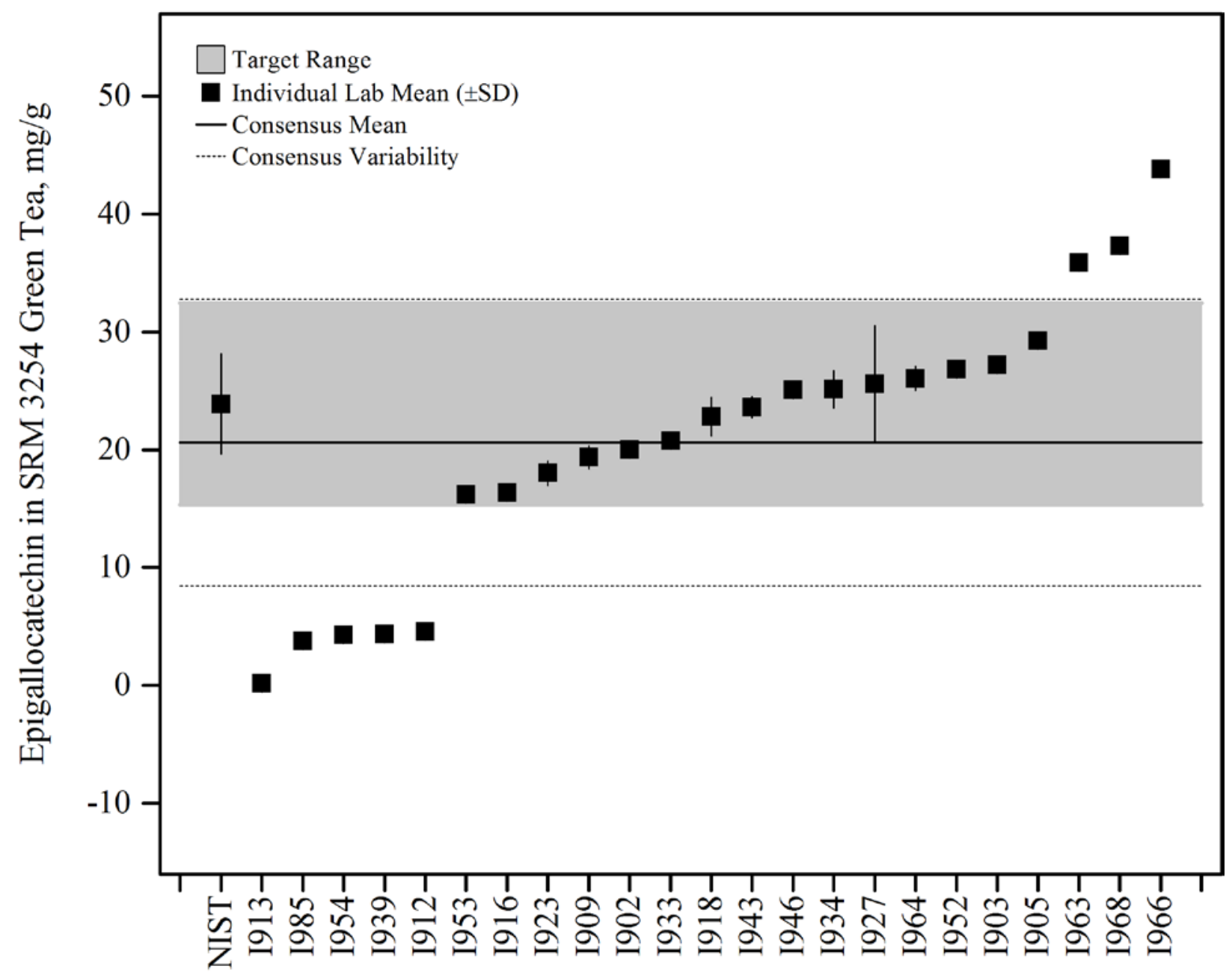

Figure 29. Epigallocatechin in SRM 3254 Camellia sinensis (Green Tea) (data summary view). In this view, individual laboratory data are plotted with the individual laboratory standard deviation (error bars). The black solid line represents the consensus mean, and the black dotted lines represent the consensus variability calculated as one standard deviation about the consensus mean. The gray shaded region represents the target zone for "acceptable” performance, which encompasses the NIST certified value bounded by twice its uncertainty $\left(U_{95}\right)$. 


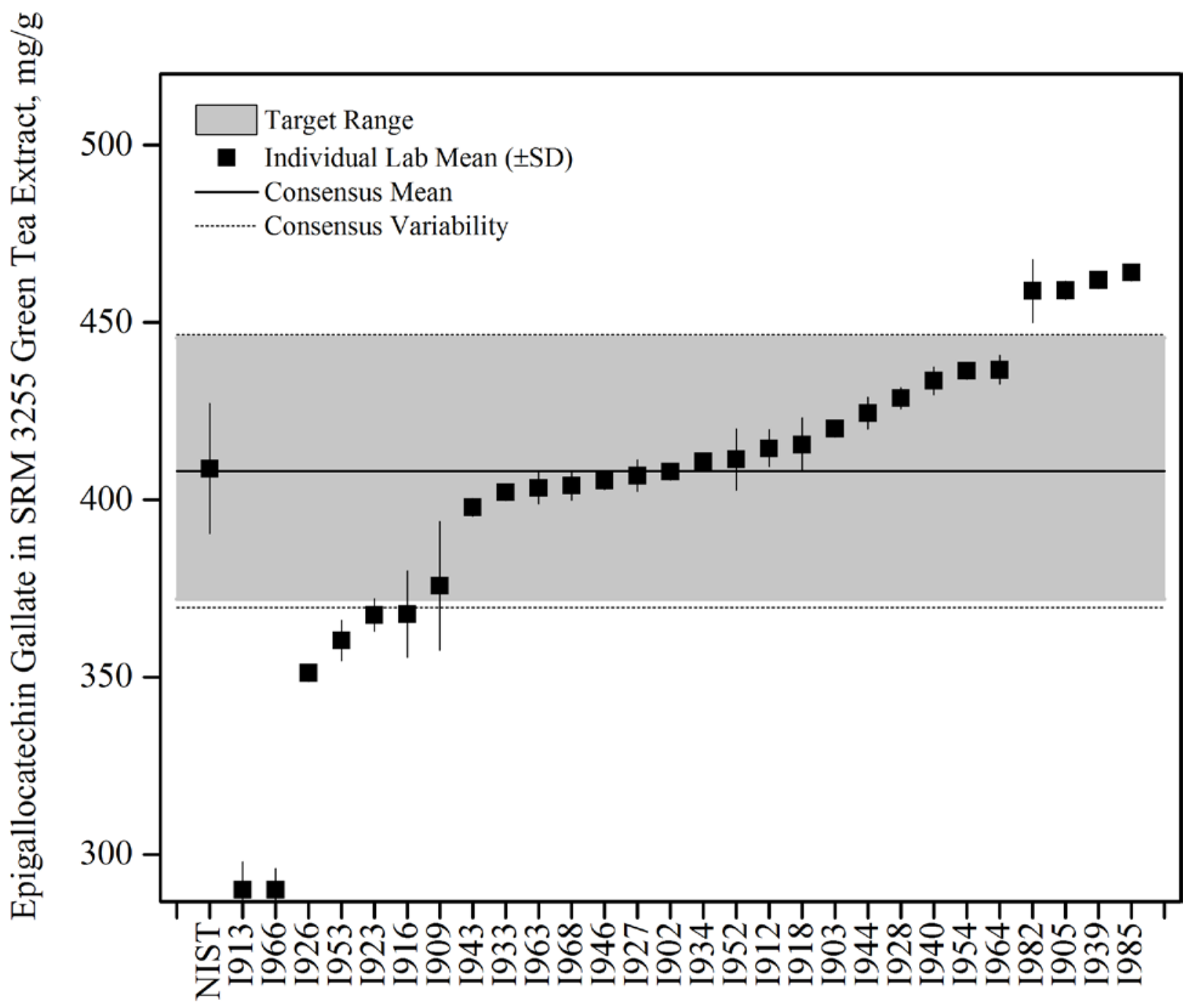

Figure 30. Epigallocatechin gallate in SRM 3255 Camellia sinensis (Green Tea) Extract (data summary view). In this view, individual laboratory data are plotted with the individual laboratory standard deviation (error bars). The black solid line represents the consensus mean, and the black dotted lines represent the consensus variability calculated as one standard deviation about the consensus mean. The gray shaded region represents the target zone for "acceptable" performance, which encompasses the NIST certified value bounded by twice its uncertainty $\left(U_{95}\right)$. 


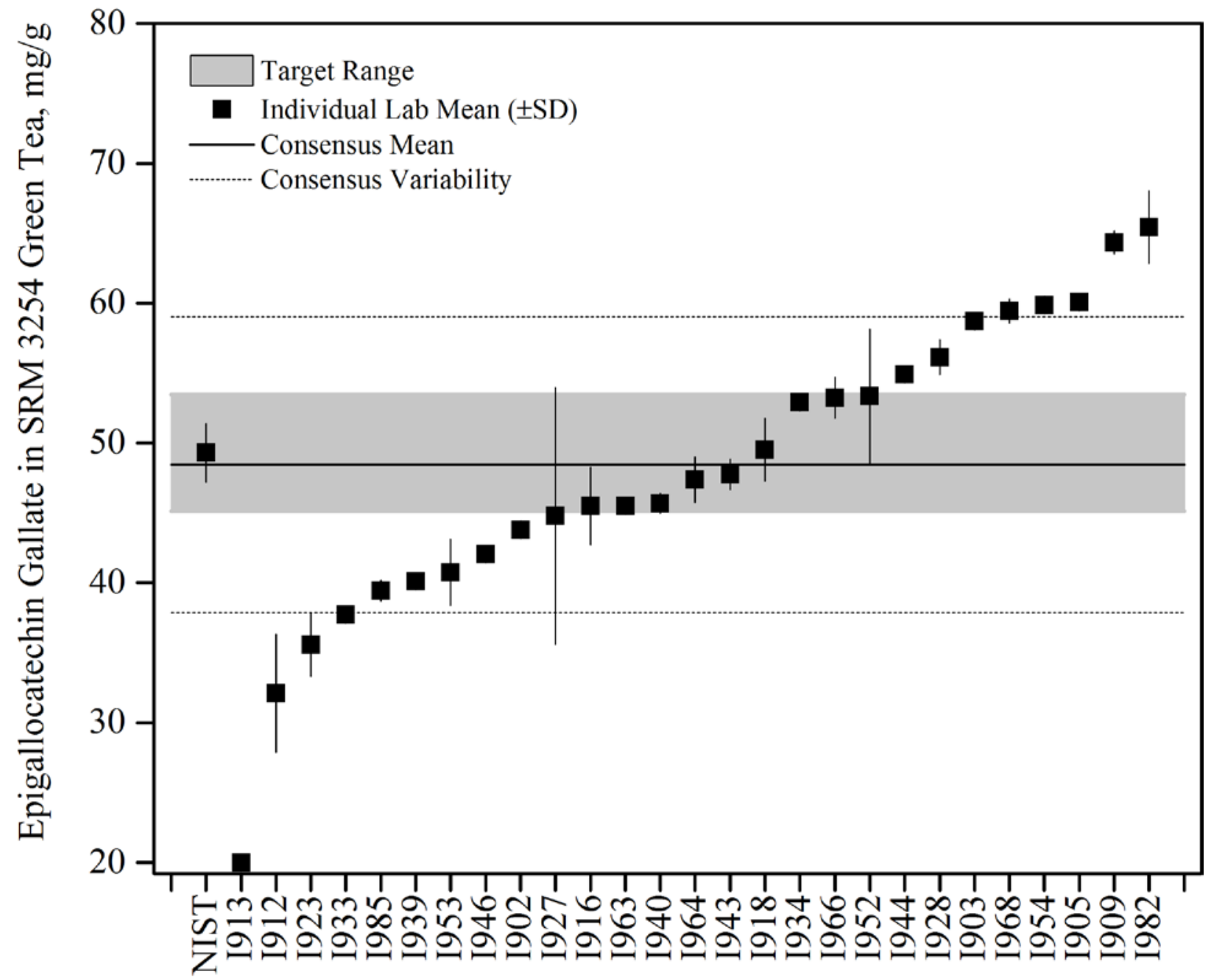

Figure 31. Epigallocatechin gallate in SRM 3254 Camellia sinensis (Green Tea) (data summary view). In this view, individual laboratory data are plotted with the individual laboratory standard deviation (error bars). The black solid line represents the consensus mean, and the black dotted lines represent the consensus variability calculated as one standard deviation about the consensus mean. The gray shaded region represents the target zone for "acceptable” performance, which encompasses the NIST certified value bounded by twice its uncertainty $\left(U_{95}\right)$. 


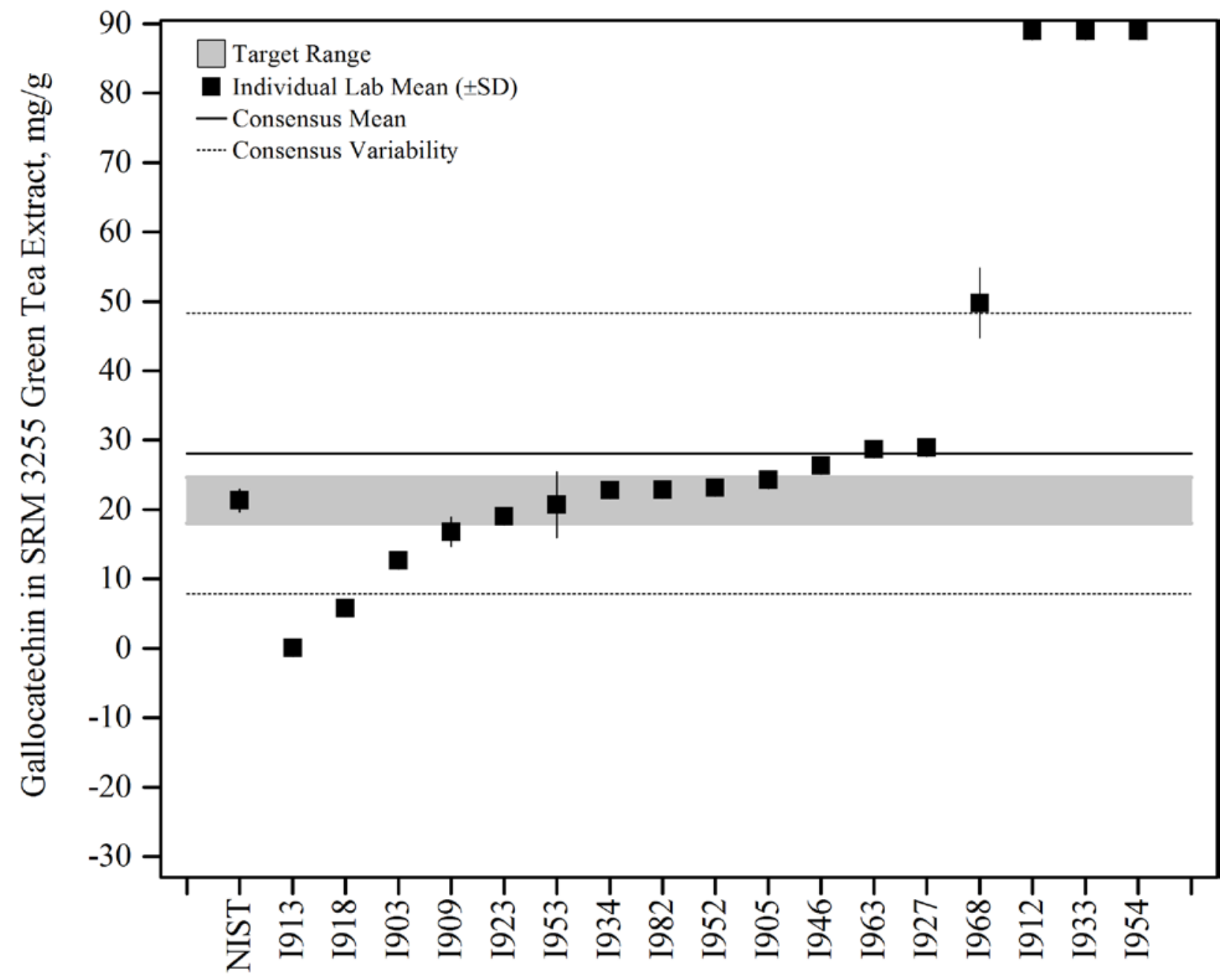

Figure 32. Gallocatechin in SRM 3255 Camellia sinensis (Green Tea) Extract (data summary view). In this view, individual laboratory data are plotted with the individual laboratory standard deviation (error bars). The black solid line represents the consensus mean, and the black dotted lines represent the consensus variability calculated as one standard deviation about the consensus mean. The gray shaded region represents the target zone for "acceptable” performance, which encompasses the NIST certified value bounded by twice its uncertainty $\left(U_{95}\right)$. 


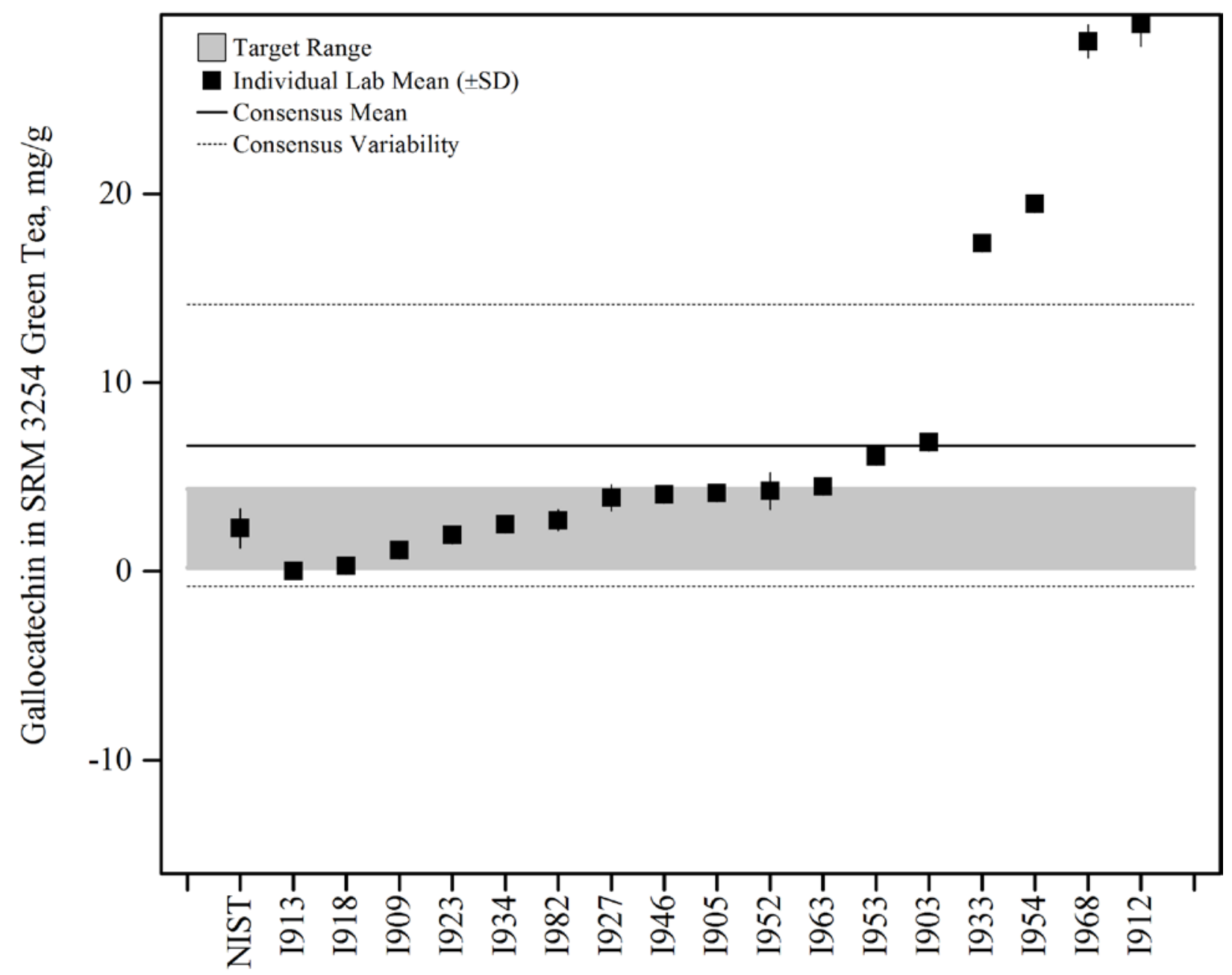

Figure 33. Gallocatechin in SRM 3254 Camellia sinensis (Green Tea) (data summary view). In this view, individual laboratory data are plotted with the individual laboratory standard deviation (error bars). The black solid line represents the consensus mean, and the black dotted lines represent the consensus variability calculated as one standard deviation about the consensus mean. The gray shaded region represents the target zone for "acceptable” performance, which encompasses the NIST certified value bounded by twice its uncertainty $\left(U_{95}\right)$. 


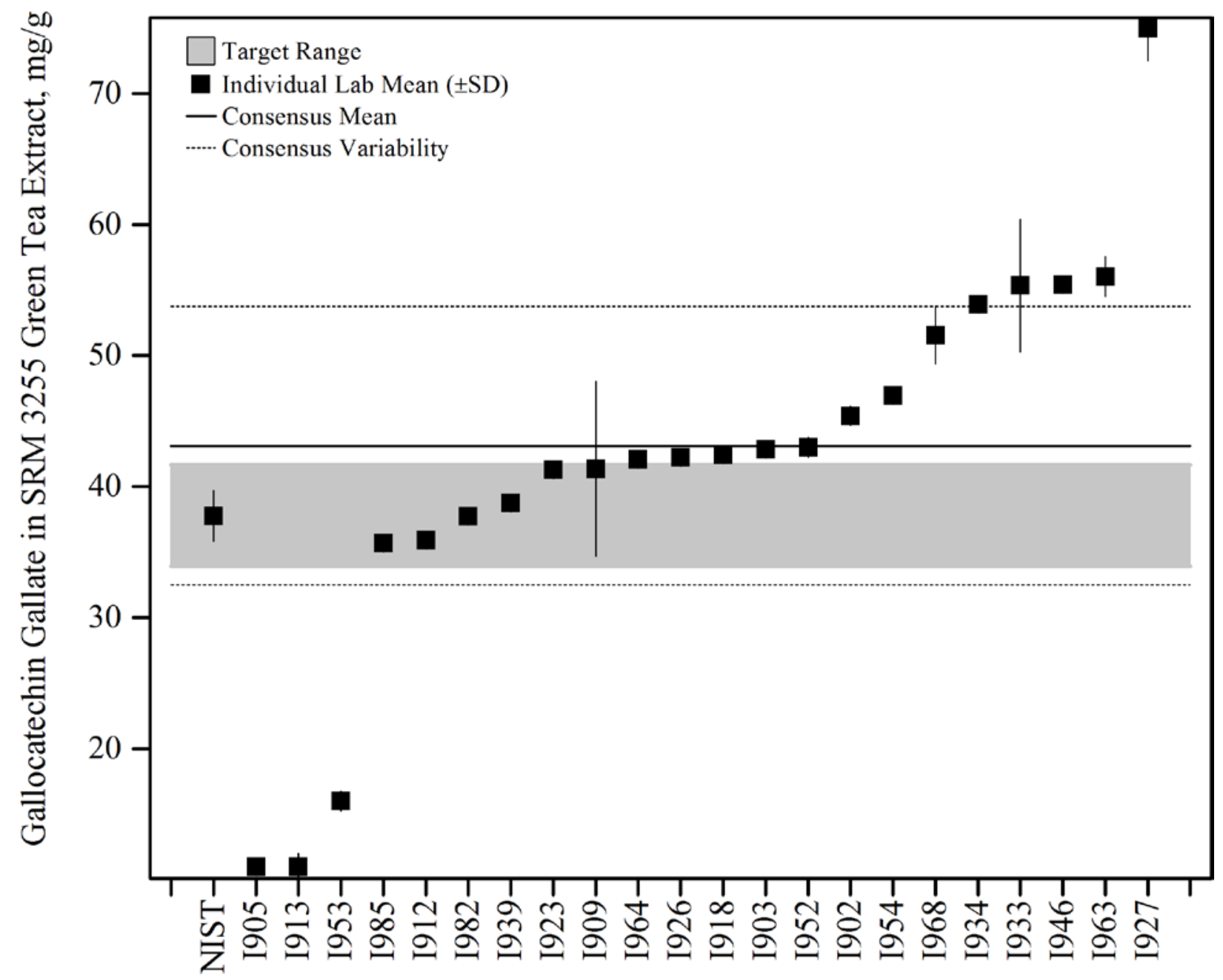

Figure 34. Gallocatechin gallate in SRM 3255 Camellia sinensis (Green Tea) Extract (data summary view). In this view, individual laboratory data are plotted with the individual laboratory standard deviation (error bars). The black solid line represents the consensus mean, and the black dotted lines represent the consensus variability calculated as one standard deviation about the consensus mean. The gray shaded region represents the target zone for "acceptable" performance, which encompasses the NIST certified value bounded by twice its uncertainty $\left(U_{95}\right)$. 


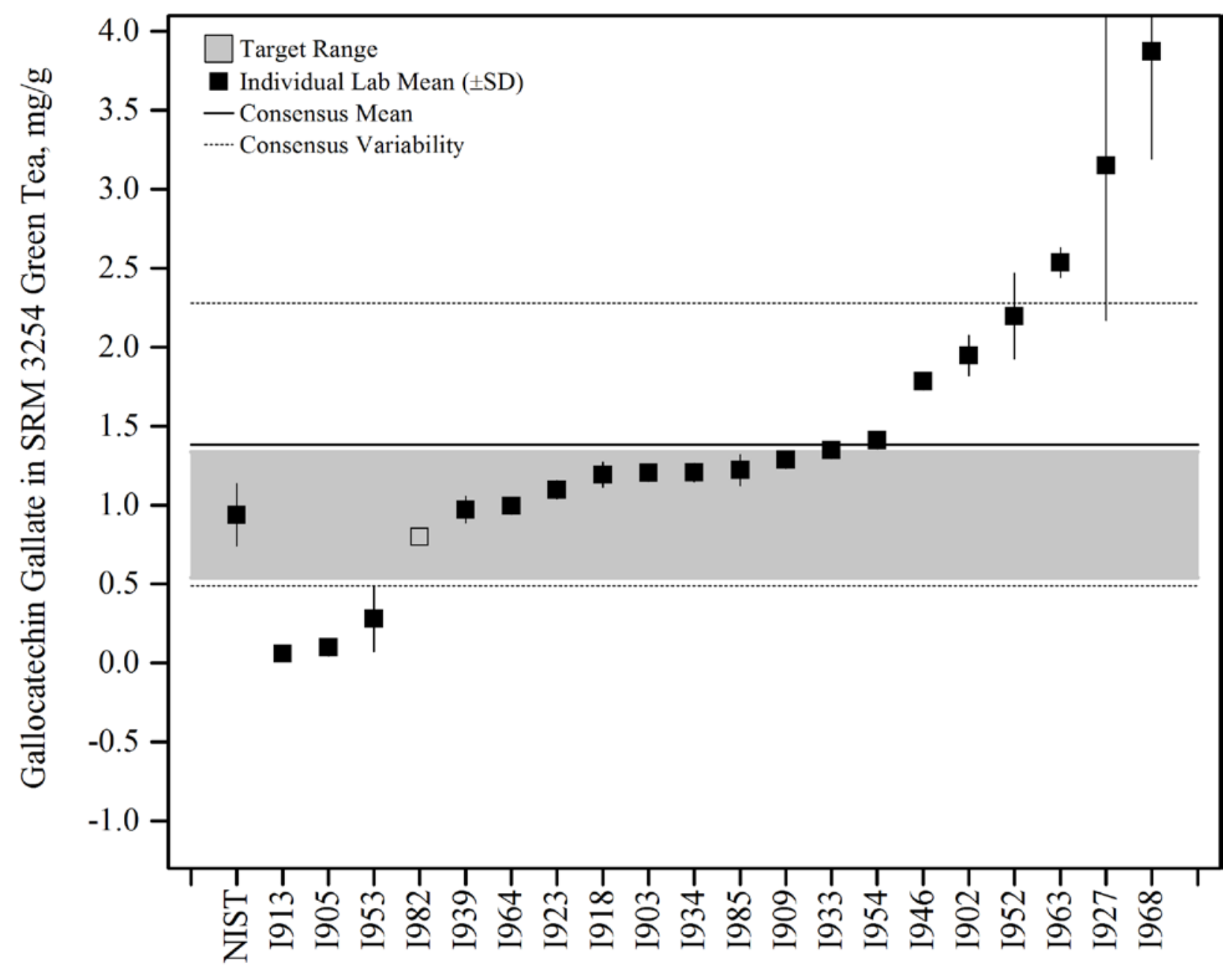

Figure 35. Gallocatechin gallate in SRM 3254 Camellia sinensis (Green Tea) (data summary view). In this view, individual laboratory data are plotted with the individual laboratory standard deviation (error bars). Data points that are unfilled represent laboratories that only reported a single value for that analyte and therefore were not included in the consensus mean. The black solid line represents the consensus mean, and the black dotted lines represent the consensus variability calculated as one standard deviation about the consensus mean. The gray shaded region represents the target zone for "acceptable" performance, which encompasses the NIST certified value bounded by twice its uncertainty $\left(U_{95}\right)$. 


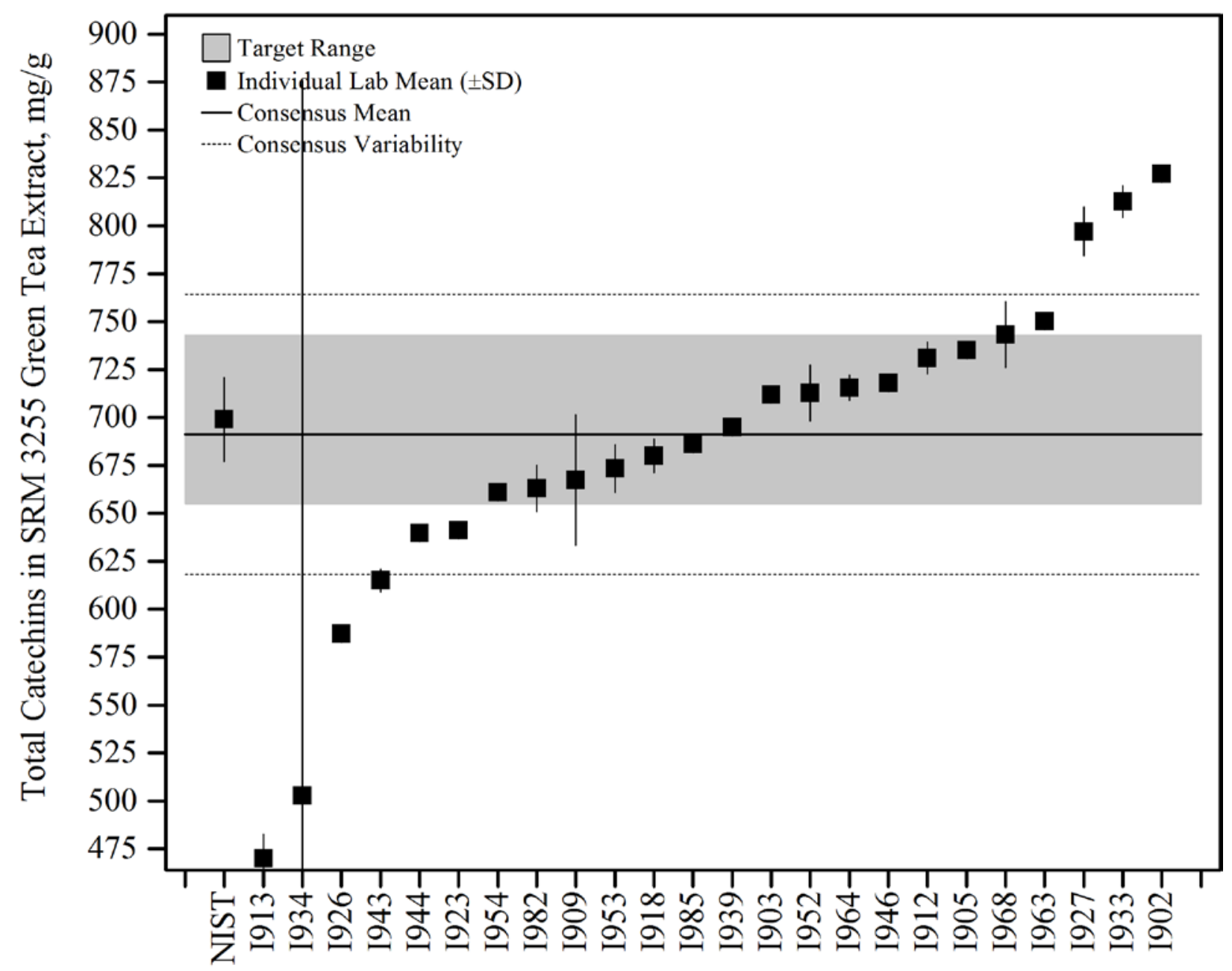

Figure 36. Total catechins in SRM 3255 Camellia sinensis (Green Tea) Extract (data summary view). In this view, individual laboratory data are plotted with the individual laboratory standard deviation (error bars). The black solid line represents the consensus mean, and the black dotted lines represent the consensus variability calculated as one standard deviation about the consensus mean. The gray shaded region represents the target zone for "acceptable” performance, which encompasses the NIST certified value bounded by twice its uncertainty $\left(U_{95}\right)$. 


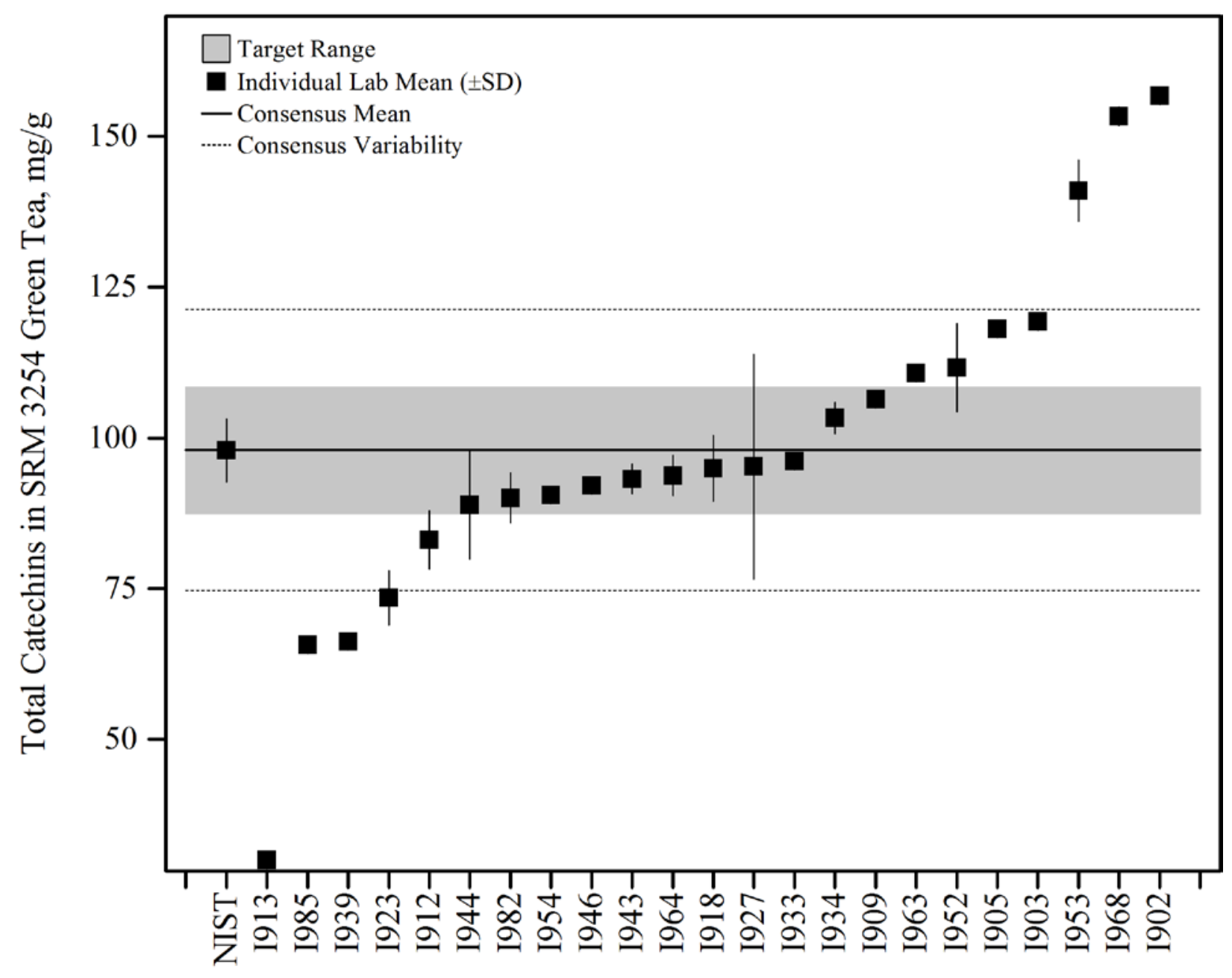

Figure 37. Total catechins in SRM 3254 Camellia sinensis (Green Tea) (data summary view). In this view, individual laboratory data are plotted with the individual laboratory standard deviation (error bars). The black solid line represents the consensus mean, and the black dotted lines represent the consensus variability calculated as one standard deviation about the consensus mean. The gray shaded region represents the target zone for "acceptable" performance, which encompasses the NIST certified value bounded by twice its uncertainty $\left(U_{95}\right)$. 


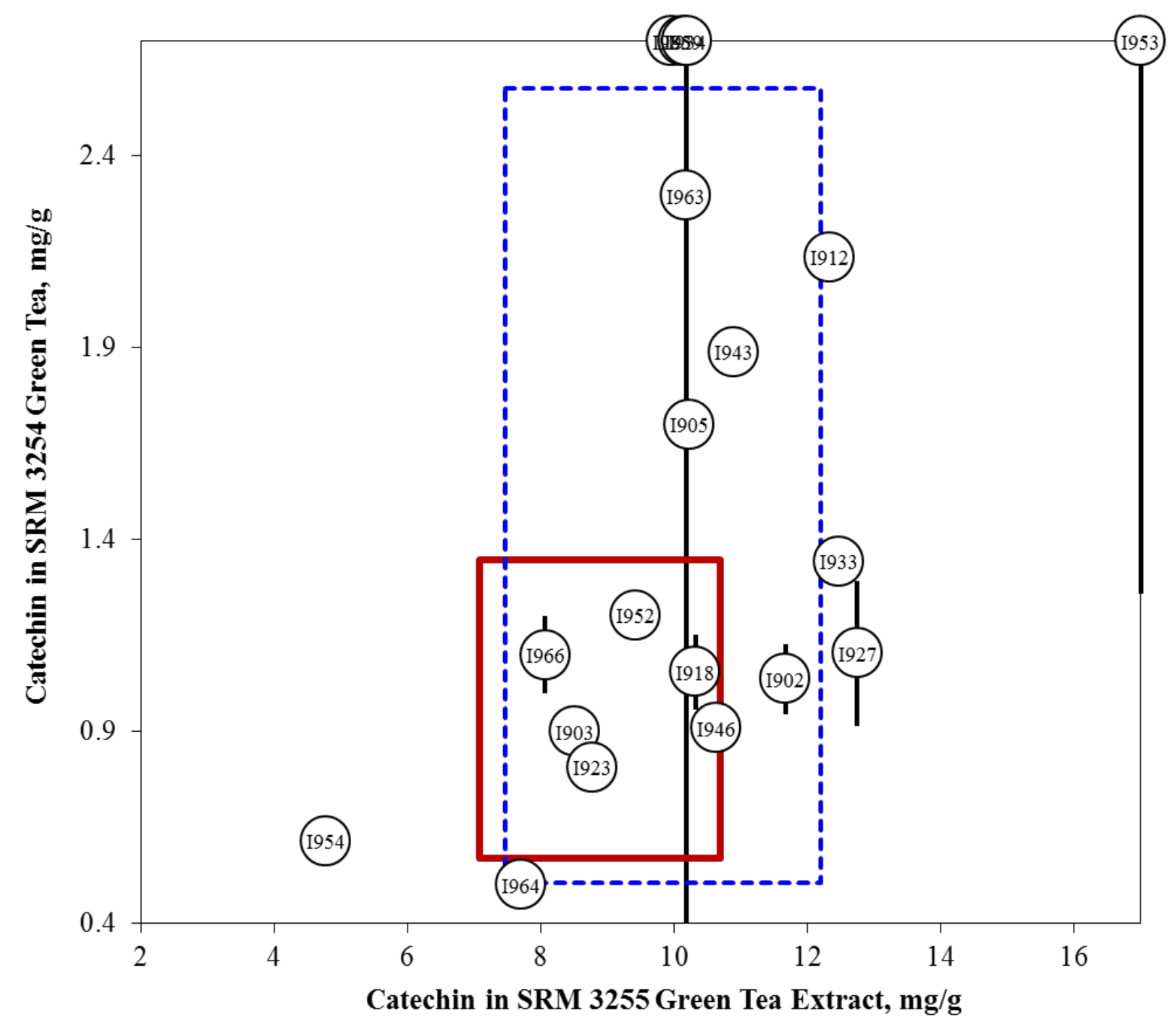

Figure 38. Catechin in SRM 3254 Camellia sinensis (Green Tea) Leaves and SRM 3255 Camellia sinensis (Green Tea) Extract (sample/control comparison view). In this view, the individual laboratory results for the control (SRM 3255 Camellia sinensis Extract) with a certified value for the analyte are compared to the results for an unknown (SRM 3254 Camellia sinensis Leaves). The error bars represent the individual laboratory standard deviation. The solid red lines represent the target zone for the control (x-axis) and the unknown sample (y-axis). The dotted blue box represents the consensus zone for the control (x-axis) and the unknown sample (y-axis). 


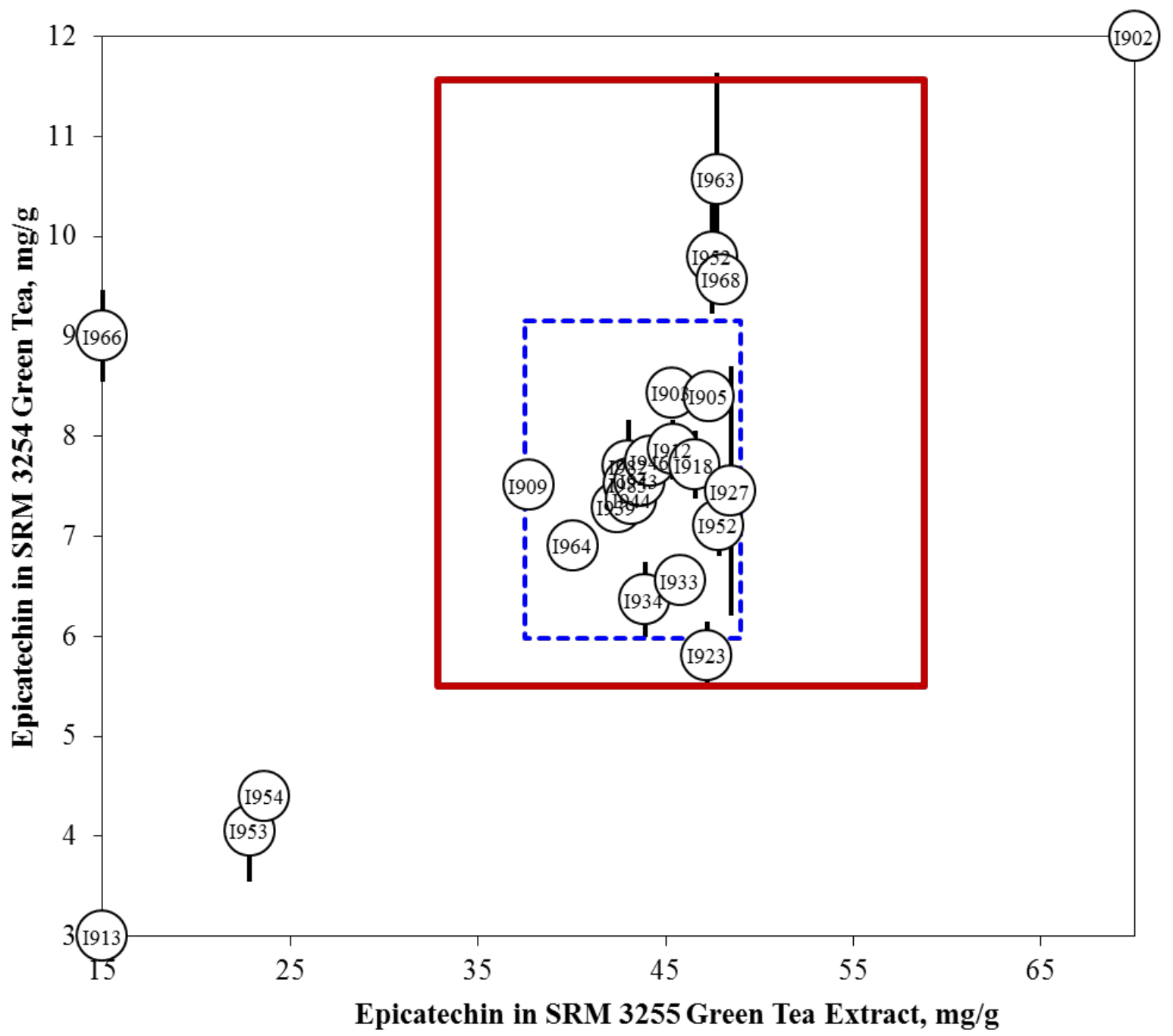

Figure 39. Epicatechin in SRM 3254 Camellia sinensis (Green Tea) Leaves and SRM 3255 Camellia sinensis (Green Tea) Extract (sample/control comparison view). In this view, the individual laboratory results for the control (SRM 3255 Camellia sinensis Extract) with a certified value for the analyte are compared to the results for an unknown (SRM 3254 Camellia sinensis Leaves). The error bars represent the individual laboratory standard deviation. The solid red lines represent the target zone for the control (x-axis) and the unknown sample (y-axis). The dotted blue box represents the consensus zone for the control (x-axis) and the unknown sample (y-axis). 


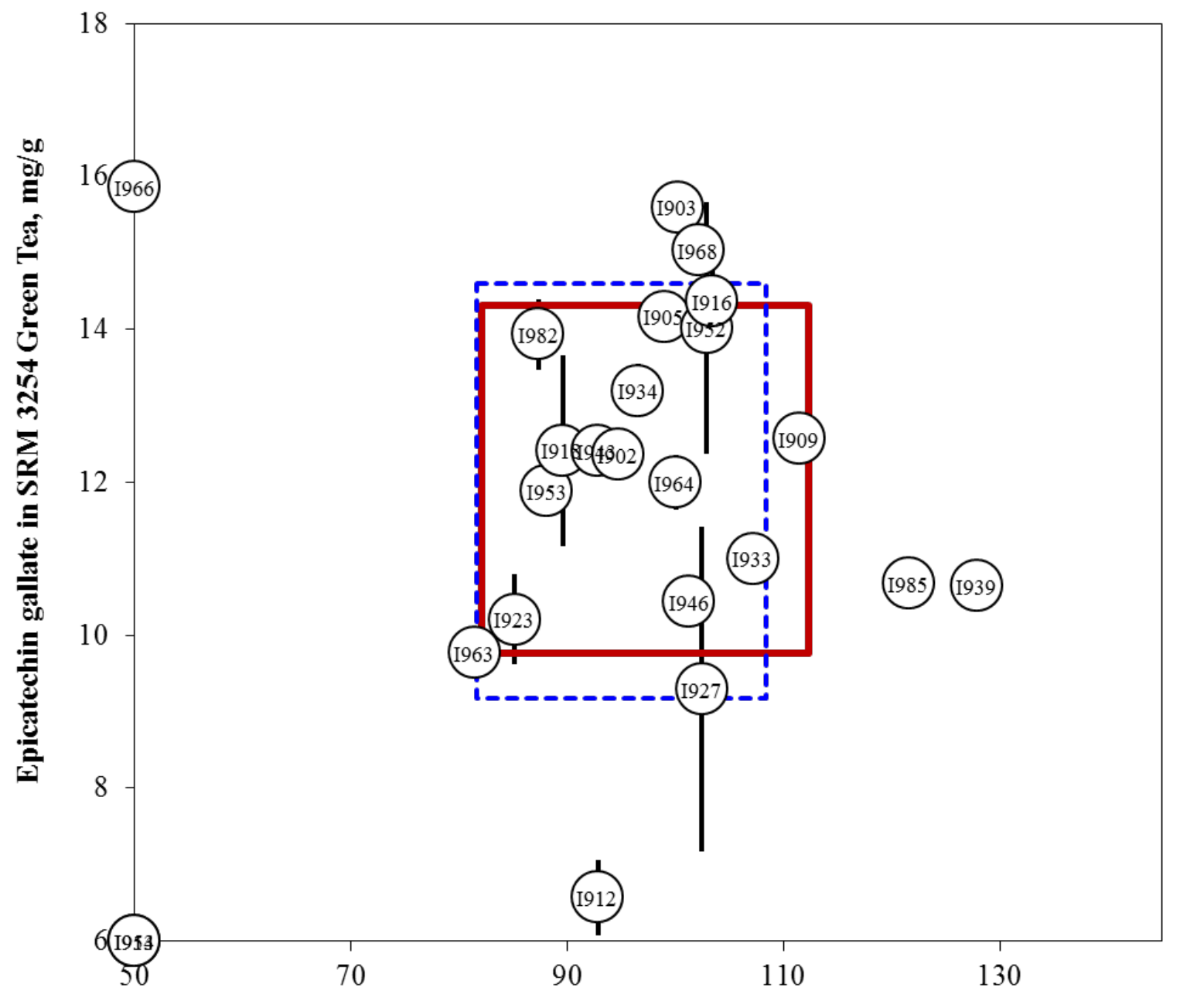

Epicatechin gallate in SRM 3255 Green Tea Extract, mg/g

Figure 40. Epicatechin gallate in SRM 3254 Camellia sinensis (Green Tea) Leaves and SRM 3255 Camellia sinensis (Green Tea) Extract (sample/control comparison view). In this view, the individual laboratory results for the control (SRM 3255 Camellia sinensis Extract) with a certified value for the analyte are compared to the results for an unknown (SRM 3254 Camellia sinensis Leaves). The error bars represent the individual laboratory standard deviation. The solid red lines represent the target zone for the control (x-axis) and the unknown sample (y-axis). The dotted blue box represents the consensus zone for the control (x-axis) and the unknown sample (y-axis). 


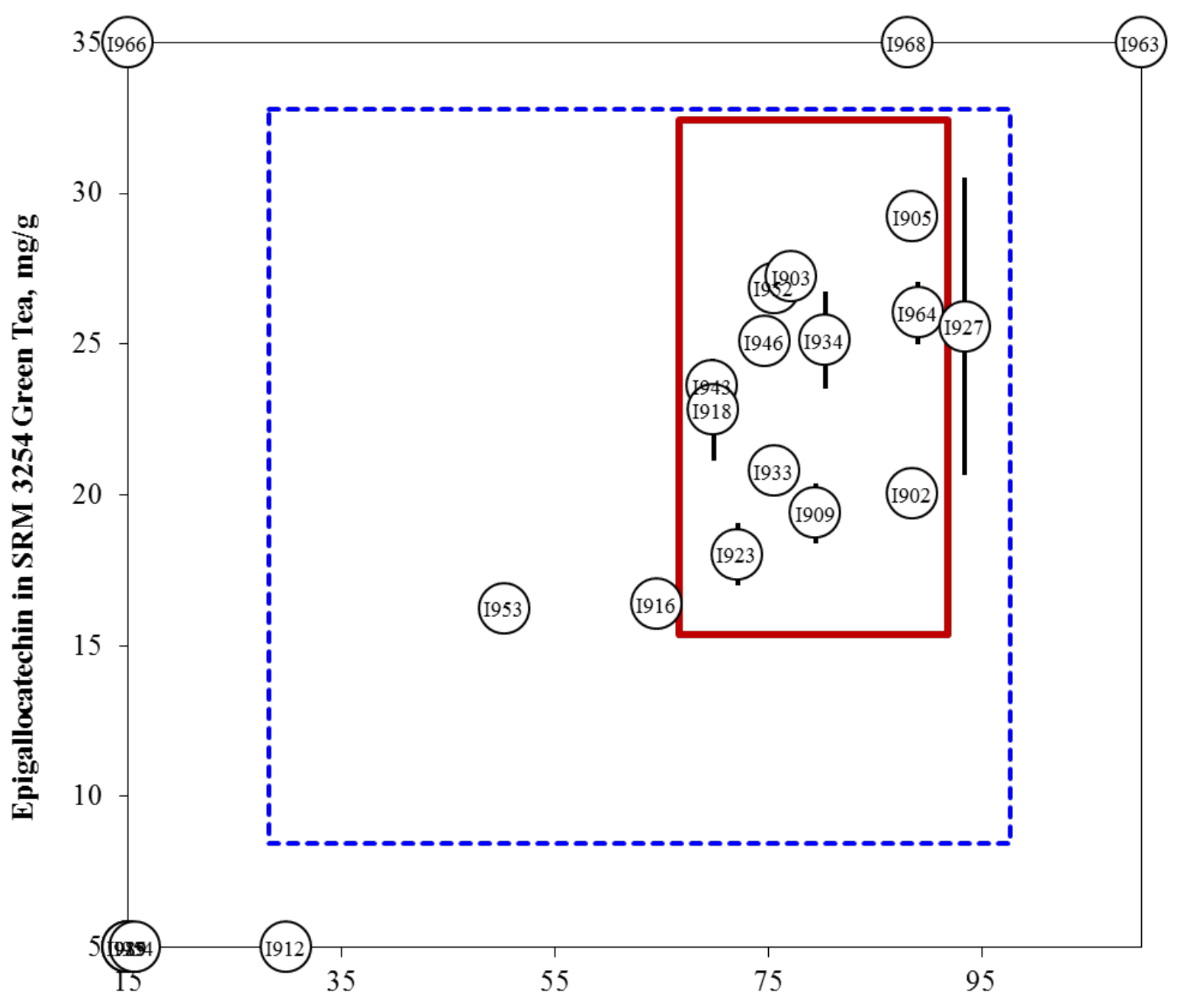

Epigallocatechin in SRM 3255 Green Tea Extract, mg/g

Figure 41. Epigallocatechin in SRM 3254 Camellia sinensis (Green Tea) Leaves and SRM 3255 Camellia sinensis (Green Tea) Extract (sample/control comparison view). In this view, the individual laboratory results for the control (SRM 3255 Camellia sinensis Extract) with a certified value for the analyte are compared to the results for an unknown (SRM 3254 Camellia sinensis Leaves). The error bars represent the individual laboratory standard deviation. The solid red lines represent the target zone for the control (x-axis) and the unknown sample (y-axis). The dotted blue box represents the consensus zone for the control (x-axis) and the unknown sample (y-axis). 


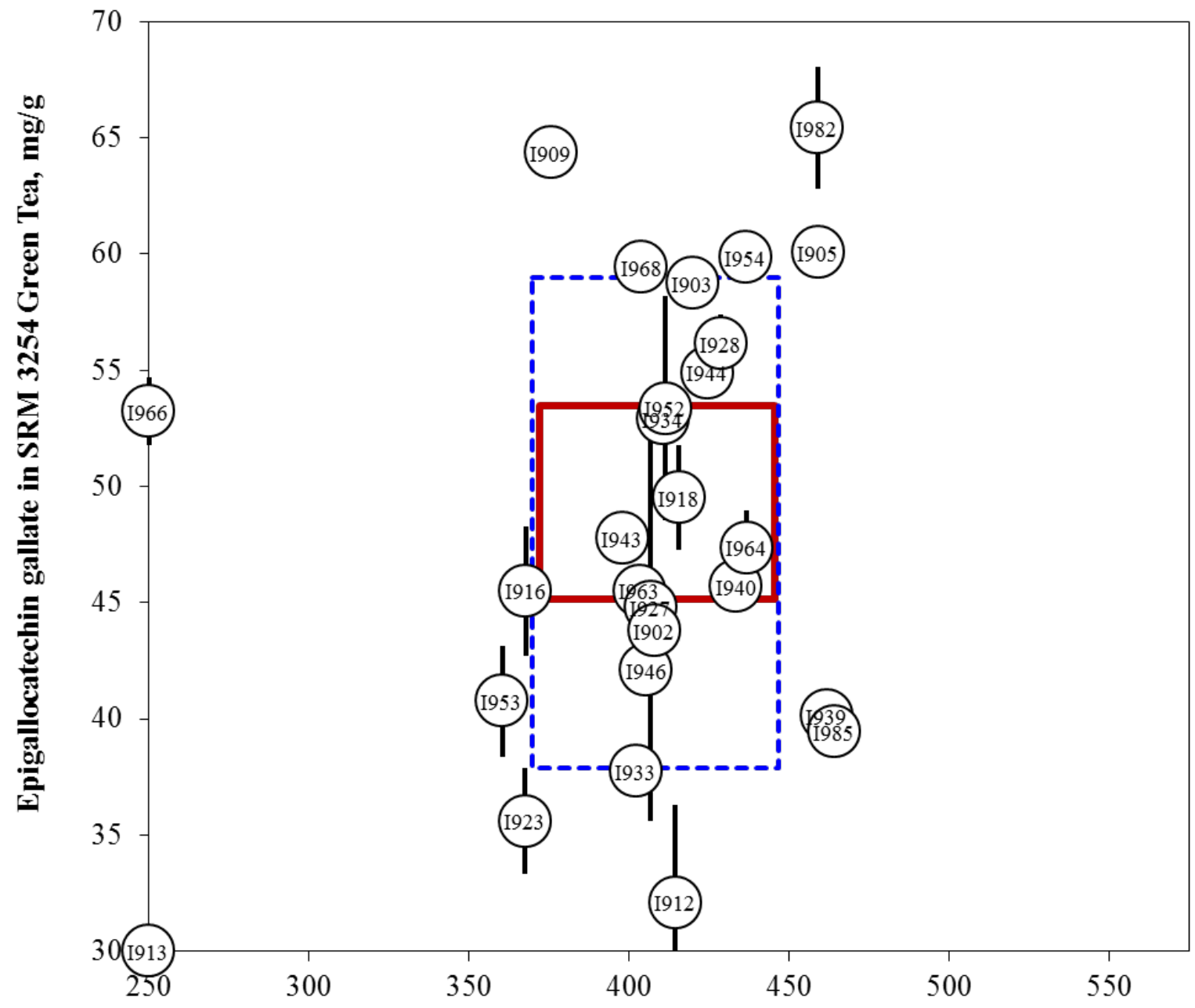

Epigallocatechin gallate in SRM 3255 Green Tea Extract, mg/g

Figure 42. Epigallocatechin gallate in SRM 3254 Camellia sinensis (Green Tea) Leaves and SRM 3255 Camellia sinensis (Green Tea) Extract (sample/control comparison view). In this view, the individual laboratory results for the control (SRM 3255 Camellia sinensis Extract) with a certified value for the analyte are compared to the results for an unknown (SRM 3254 Camellia sinensis Leaves). The error bars represent the individual laboratory standard deviation. The solid red lines represent the target zone for the control (x-axis) and the unknown sample (y-axis). The dotted blue box represents the consensus zone for the control (x-axis) and the unknown sample (y-axis). 


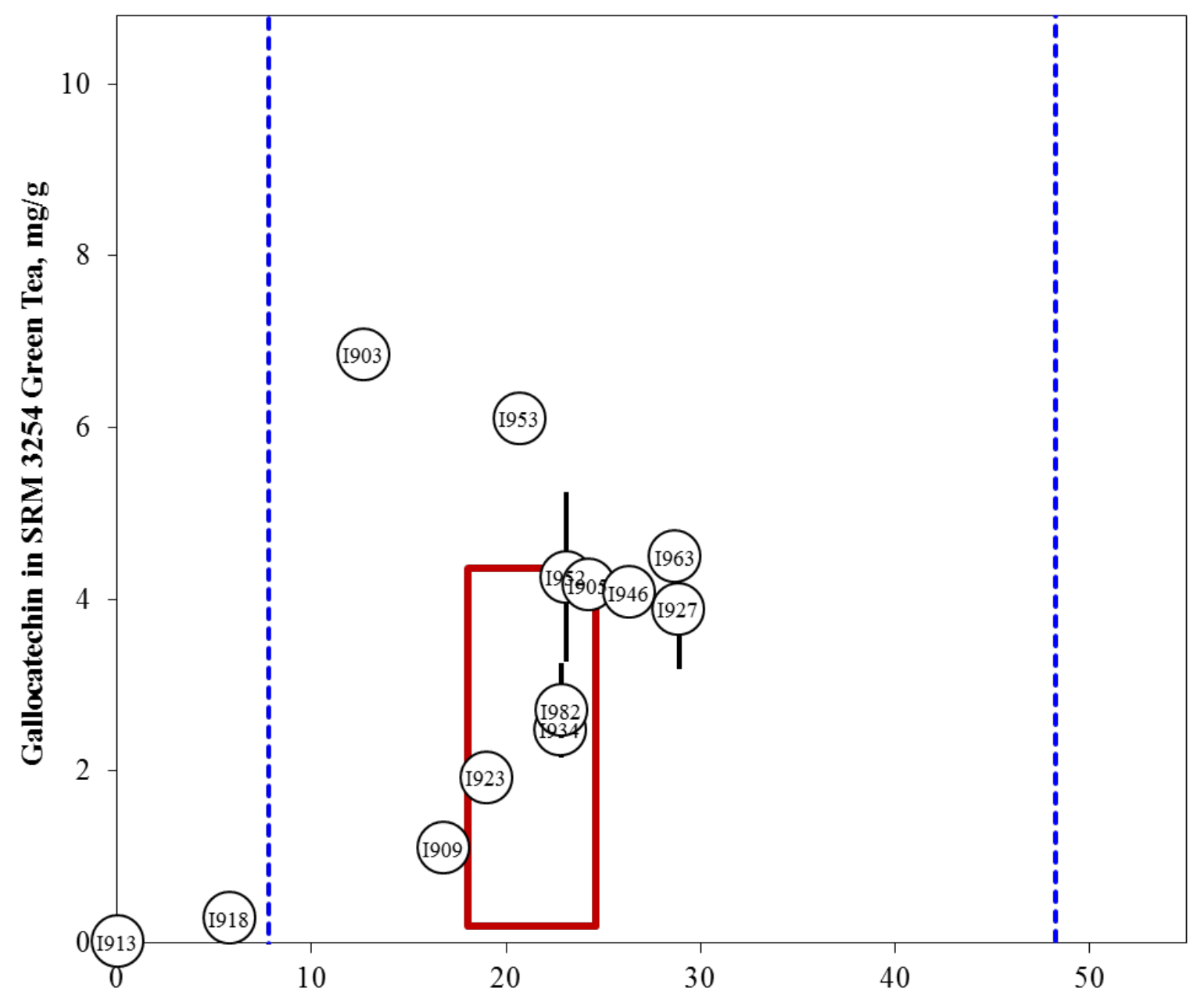

Gallocatechin in SRM 3255 Green Tea Extract, mg/g

Figure 43. Gallocatechin in SRM 3254 Camellia sinensis (Green Tea) Leaves and SRM 3255 Camellia sinensis (Green Tea) Extract (sample/control comparison view). In this view, the individual laboratory results for the control (SRM 3255 Camellia sinensis Extract) with a certified value for the analyte are compared to the results for an unknown (SRM 3254 Camellia sinensis Leaves). The error bars represent the individual laboratory standard deviation. The solid red lines represent the target zone for the control (x-axis) and the unknown sample (y-axis). The dotted blue box represents the consensus zone for the control (x-axis) and the unknown sample (y-axis). 


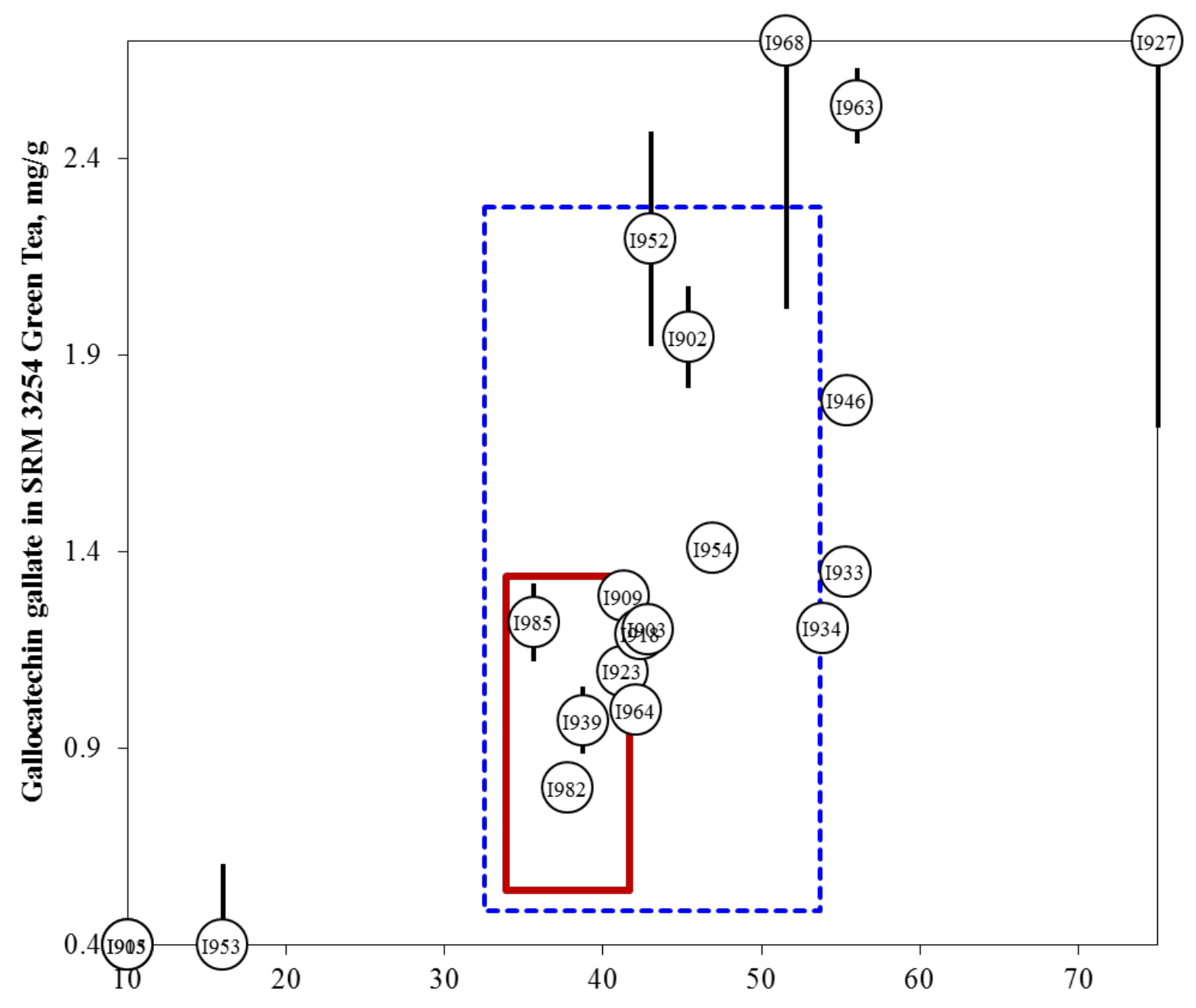

Gallocatechin gallate in SRM 3255 Green Tea Extract, mg/g

Figure 44. Gallocatechin gallate in SRM 3254 Camellia sinensis (Green Tea) Leaves and SRM 3255 Camellia sinensis (Green Tea) Extract (sample/control comparison view). In this view, the individual laboratory results for the control (SRM 3255 Camellia sinensis Extract) with a certified value for the analyte are compared to the results for an unknown (SRM 3254 Camellia sinensis Leaves). The error bars represent the individual laboratory standard deviation. The solid red lines represent the target zone for the control (x-axis) and the unknown sample (y-axis). The dotted blue box represents the consensus zone for the control (x-axis) and the unknown sample (y-axis). 


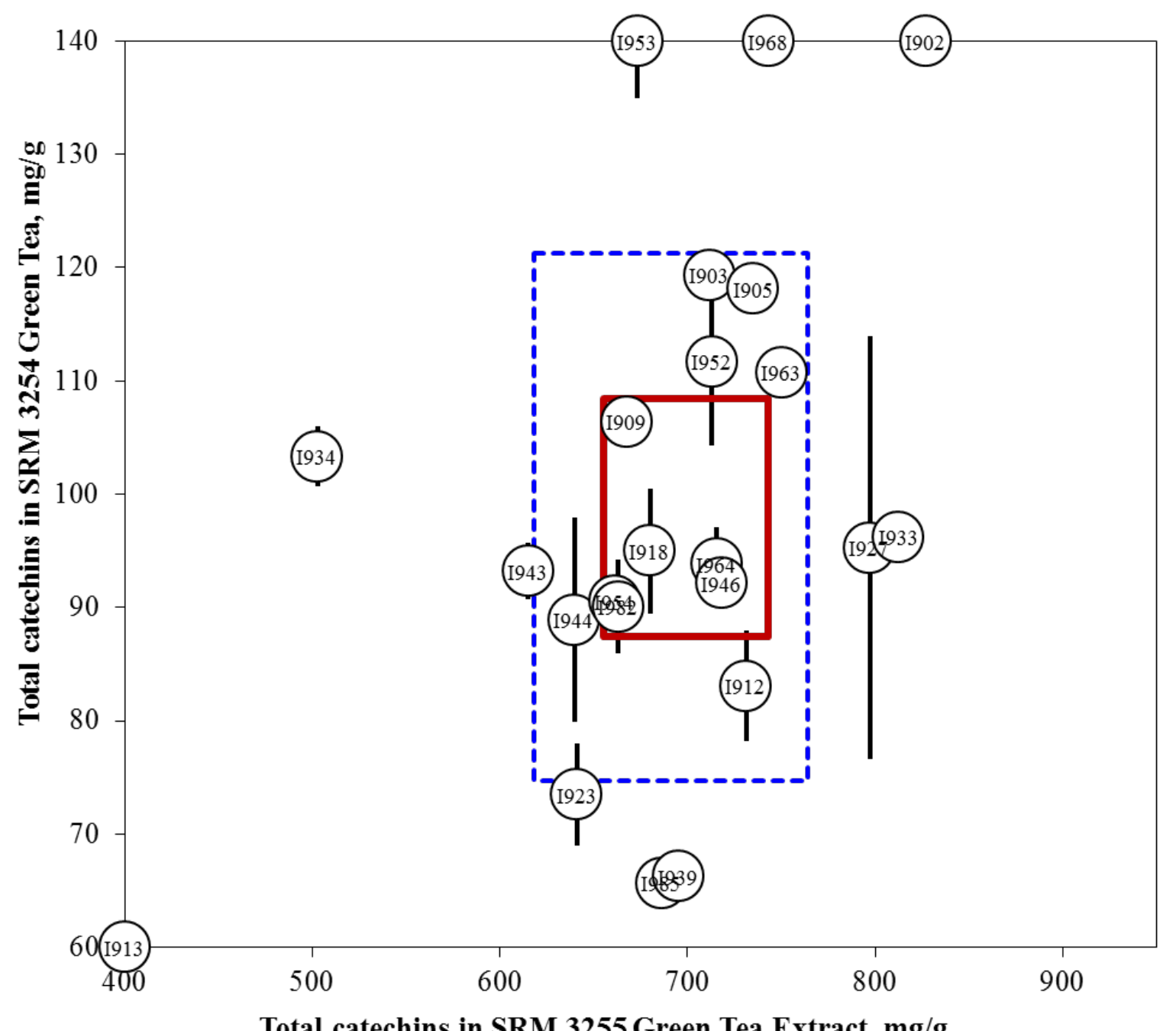

Total catechins in SRM 3255 Green Tea Extract, mg/g

Figure 45. Total catechins in SRM 3254 Camellia sinensis (Green Tea) Leaves and SRM 3255 Camellia sinensis (Green Tea) Extract (sample/control comparison view). In this view, the individual laboratory results for the control (SRM 3255 Camellia sinensis Extract) with a certified value for the analyte are compared to the results for an unknown (SRM 3254 Camellia sinensis Leaves). The error bars represent the individual laboratory standard deviation. The solid red lines represent the target zone for the control (x-axis) and the unknown sample (y-axis). The dotted blue box represents the consensus zone for the control (x-axis) and the unknown sample (y-axis). 